\title{
SOIL-CORROSION STUDIES, 1941: FERROUS AND NON- FERROUS CORROSION-RESISTANT MATERIALS AND NONBITUMINOUS COATINGS
}

\author{
By Kirk H. Logan and Melvin Romanoff
}

\section{ABSTRACT}

The soil-corrosion investigation started in 1922 was intended to yield information on the corrosiveness of typical soils throughout the United States. The investigation of special materials and coatings begun 10 years later was intended primarily to assist manufacturers in the development of materials suitable for use in corrosive soils.

No attempt to secure specimens such that systematic data on the effect of individual alloying elements could be obtained was made but, manufacturers were invited to submit materials on which they desired information. Furthermore, the specimens were buried under simulated practical conditions, so that accurate technical control was not feasible. Also, since very few specimens of each type were included, dispersion of the data resulting from the general conditions of the tests makes it impossible to draw very definite conclusions with regard to the comparative merits of the several materials investigated. Nevertheless, the data give a general indication of what may be expected of a considerable variety of alloys when exposed to severe soil conditions. The addition of very small percentages of alloying elements does not have a marked effect on the rate of corrosion, but rather large percentages are apt to bring about a considerable improvement in corrosion resistance.

Steels high in nickel and chromium, and copper alloys high in copper are very resistant to nearly all soil conditions. Lead corrodes only slightly when a coating of an insoluble lead salt is deposited on the lead.

Bursting and crushing tests indicate that asbestos-cement pipe gained somewhat in strength from exposure to the soil for 4 years.

A 3-ounce coating of zinc adds about 3 years to the life of steel exposed to some of the most corrosive soils in the test sites. Lead coatings appear to be inadequate for severe soil conditions.

Several thick experimental coatings prevented practically all corrosion at most of the test sites for 9 years. Air-dried Bakelite coatings blistered within 4 years, but a baked-on Bakelite coating showed no change in appearance after 4 years except for a few blisters. Pitting occurred under some of these blisters. Most thin coatings blistered, became brittle, and within 4 years permitted rusting and pitting of the metal to which they were applied.

\section{CONTENTS}

I. Introduction

II. Properties of the soils at the test sites

III. Ferrous materials

1. Cast materials

(a) Dimensions and composition

(b) Specimens exposed for 9 years

(c) Specimens exposed for 2 years

2. Wrought materials

(a) Dimensions and composition

(b) Specimens exposed for 9 years

1. Specimens of pipe

2. Specimens of high-alloy sheet

(c) Specimens of alloy plates exposed for 4 years

(d) Specimens exposed for 2 years 
Page

IV. Copper and copper-alloy

1. Dimensions and composition

2. Specimens exposed for 9 years

3. Muntz metal specimens exposed for 2 years

V. Zinc

VI. Lead _ _ $\ldots \ldots \ldots \ldots$

VII. Asbestos-cement pipe__. 183

1. Water-absorntion test.

2. Crushing tests .

3. Bursting tests

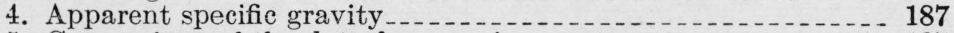

5. Comparison of the data from various tests

VIII. Metallic Coatings _._. 188

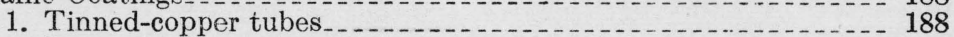

2. Lead

3. Zinc

IX. Nonbituminous organic coatings _... 190

1. Coatings exposed for 9 years $\ldots \ldots \ldots \ldots 1$

2. Coatings exposed for 4 years

3. Coatings exposed for 2 years

X. Summary

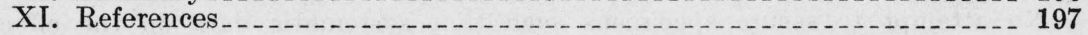

\section{INTRODUCTION}

The studies of the effects of soils on materials used for underground pipes which the Bureau began in 1922, indicated that at least for the periods of exposure covered by the investigation, the maximum penetration of the materials by corrosion was approximately the same for the different commonly used ferrous materials [1]. ${ }^{1}$ Some ferrous alloys and some nonferrous alloys and metals appeared to be more resistant to the corrosive action of soils [2]. The investigation indicated also that rapid corrosion of the commonly used ferrous materials occurred under several soil conditions, and the conclusion [3] was reached that with respect to these materials the seriousness of the corrosion was largely controlled by the soil conditions to which the materials were exposed and nearly independent of the way in which the materials were made. It was shown that under most soil conditions the rate of maximum penetration of the metal decreased as the period of exposure increased [4]. The change in rate was attributed to changes in aeration and moisture as the soil in the trench settled, to a possible depletion of the corrosive elements in the soil adjacent to the specimens, and to the protective effect of the corrosion products.

Concurrent studies of bituminous protective coatings $[5,6,7]$ indicated that this means of preventing corrosion was not entirely satisfactory partly because of the characteristics of the materials used and because of the difficulty of maintaining a complete and uninjured coating under the conditions to which it was usually subjected while the pipe line was being laid.

The original investigation [8] was, for the most part, limited to commonly used pipe materials and was intended to give comprehensive results on their reactions to typical soils throughout the United States. The results of this investigation stimulated an increased interest in the problem of discovering more effective ways of combating the destructive action of severely corrosive soils. A new investigation was undertaken in 1932 to study the effectiveness of

\footnotetext{
1 Figures in brackets indicate the literature references at end.of this paper.
} 
corrosion-resisting pipe materials and the application of improved protective coatings. Fifteen different soils, most of which were known to be corrosive with respect to the commonly used irons and steels, were selected, and manufacturers were invited to submit specimens of materials which they believed or hoped would be resistant to the chosen soil conditions.

Since no attempt was made to secure specimens such that systematic data on the effect of individual alloying elements or their amounts could be obtained, the samples submitted differed in so many ways that in many cases it is impossible to explain observed differences in behavior. The mill scale was left on some of the materials because this condition represented the way the materials were normally used. Other materials were free from scale. There is some evidence that the mill scale affected the depth and distribution of the pits. In most cases the materials differed in more than one alloying element. In one case several materials differing in composition, and hence in electrical potential, were connected together to represent a common field condition. Some of the alloys and coatings were experimental. Others are at this time too expensive for ordinary underground use. Some commonly used pipe materials were buried as controls.

The above explanation should indicate to the reader that the term "corrosion resistant" may be inaccurate with respect to some of the materials reported on, and that the results of the tests do not represent the corrosiveness of soils in general.

It will be shown that some materials which corrode rapidly under some soil conditions are quite resistant to other corrosive soils.

Why pipes corrode when buried and why they corrode more in some soils than in others has not been fully determined. The commonly assigned cause of underground corrosion is a difference of potential between various points on the surface of the material. This difference may arise from lack of homogeneity of the metal, that is, the presence of mill scale, segregations, or the exposure of two different components of the materials, or from differences in the soil in contact with the material at different places. Soil solutions are usually too weak to account directly for the corrosion observed under most soil conditions. However, the electrical conductivity of the soils containing considerable quantities of soluble salts is an important factor in corrosion. Soil bacteria [9] have been shown to be a direct or indirect cause of corrosion in some soils.

The potential differences referred to are such that many investigators of corrosion have devoted most of their efforts to discovering why corrosion is not more serious. Their explanations [10,11, 12, 13] deal largely with the character and distribution of corrosion products and with polarization resulting from the flow of corrosion currents.

The results of the corrosion tests reported in this paper should be interpreted with care because the conditions to which the specimens were exposed cannot be identical with those to which pipes of the materials tested may be subjected, because other conditions affecting the pipe may retard or increase the rate of corrosion, and because a pit of any chosen depth is more likely to be found on a large area (as that of a long pipe) than on a small one of the same material exposed to similar conditions. 


\section{Journal of Research of the National Bureau of Standards}

The life of a pipe [14] can be estimated only indirectly and very roughly from the data to be presented, and that estimate will represent only the average life of a large number of pipes. The life of any individual pipe may be quite different from the average, just as the life of a person may be quite different from that of the average individual, as given in life-expectancy tables. Lack of exact reproducibility is characteristic of underground corrosion, especially with respect to pit depths, and in the discussion of the data presented in this paper attention will be called to instances in which the total pit depth for the period under discussion is less than the corresponding pit depth for a shorter period of exposure under nominally the same conditions. Final conclusions as to the corrodibility of the materials should therefore be withheld until all the specimens have been removed, which may be several years after the close of the present war. Nevertheless, the data are of considerable interest and value in that they indicate roughly the probable relative merits of several materials under several soil conditions and show the effects of the addition of alloying elements and suggest possibly helpful changes in the composition of pipe materials.

Conclusions based on the data on protective coatings should be drawn with even greater caution, since these coatings were applied under laboratory conditions that were probably much more favorable to good application than under commercial conditions, and because the coated specimens were handled with much greater care than it is practical to use with coated pipes. It is much easier to produce a perfect coating on a short piece of small-diameter pipe than on a long length of large-diameter pipe, and a coated-pipe line is subjected to destructive conditions not encountered by a small coated specimen. However, the data are of value for comparative purposes and indicate that considerable progress has been made in the art of pipe-line protection.

Perhaps a word of caution should be added regarding the use of averages with respect to underground corrosion. When a number of observations of the same thing are made, the results will not be identical because of imperfections in the methods and apparatus used. They should, however, be nearly alike, and an average of the results affords the best information concerning the measurements. However, when different things are measured, such as pit depths on the same materials in different soils, the average of the results yields little information as to what may be expected under any specific condition. For this reason such statements as the average life of pipe in the United States are of little value except from a statistical standpoint. For specific information, the performance of the material under consideration with respect to the soil conditions to be encountered must be known. Often these data are not available. In such cases analogous data may be considered, but it is seldom wise to use averages of data all of which were not obtained under the same conditions.

This report presents data on ferrous and nonferrous pipe materials and on nonbituminous pipe coatings removed in 1941 after exposures of approximately 2, 4, and 9 years. Other specimens of all but the 2-year-old materials have been removed and reported on in earlier publications $[1,2,3,15,16,17]$, and still others will be removed in the future. 


\section{PROPERTIES OF THE SOILS AT THE TEST SITES}

As the character of the soil is the controlling factor in underground corrosion, information as to the characteristics of the soils at the test sites is essential to an understanding of the report. Most of this section of the paper is a reproduction of a similar section in Research Paper RP1460 [2]. Since that paper was written, it has been found that the $\mathrm{pH}$ values of certain soils when in their undisturbed condition are not the same as those of samples of the same soils after they have been aerated for some time [18]. The $\mathrm{pH}$ values given in table 1 were determined after the samples had been dried and pulverized, which is standard practice in the determination of the $\mathrm{pH}$ values of soils. When conditions permit, the $\mathrm{pH}$ values of all soils in the Bureau of Standards soil-corrosion investigations will be redetermined using soil in its natural condition. It is probable that this will result in some changes in the reported values for certain of the poorly aerated soils, since it has been found that aeration of these soils tends to lower their $\mathrm{pH}$ values. Attention is also called to the fact that the different soil horizons, or layers, sometimes differ widely in their physical and chemical properties, and hence in their corrosiveness. It follows that the data in this report are strictly applicable only to pipes buried in the soil horizons to which the specimens were exposed. In some soils, such as Rifle peat (soil 60 ), the peaty $B$ horizon is underlain by a layer of clay at a depth of from 20 to 30 inches, and the two layers differ greatly in corrosiveness. Frequently large-diameter pipes are exposed to two soil horizons, and it seems probable that corrosion is accelerated because of a difference of potential between these horizons. Corrosion of large-diameter pipes may also be accelerated by differences in the aeration of the bottom and top of the pipe. It has been noted that the maximum pits frequently occur near the bottom of the pipe, where the aeration is poorest.

The nature of the soils at the test sites is indicated by the physical and chemical properties shown in table 1. The texture of the soils and their retentiveness of water is indicated relatively by values for the moisture equivalent, the quantity of water retained by a previously saturated soil against a centrifugal force of 1,000 times gravity. Since the true specific gravity of the mineral portion of soils varies within narrow limits, the apparent specific gravity, except in the case of organic soils, can be taken as a measure of their compactness and hence as a relative measure of their porosity. A soil having a very high moisture equivalent and a high apparent specific gravity, such as Acadia clay (soil 51), may be considered to be very fine in texture, highly retentive of water, very dense, and impermeable to the flow of air and water; this is confirmed by the aeration or drainage of the soil, which is poor. On the other hand, the fairly large value for the moisture equivalent (32 percent) of Hagerstown loam (soil 55), indicates this soil to be fairly heavy in texture and retentive of water. However, it is also very porous and well aerated, as indicated by the low value of its apparent specific gravity, 1.49.

Consideration of the chemical properties given in table 1 shows that the test sites represent a wide range of soil conditions. The range in $\mathrm{pH}$ is from 2.6 to 9.4 , approximately the extreme limits shown by soils. The resistivity ranges from 62 to approximately $18,000 \mathrm{ohm}-\mathrm{cm}$, corresponding to the concentration of sea water, on the one hand, to 


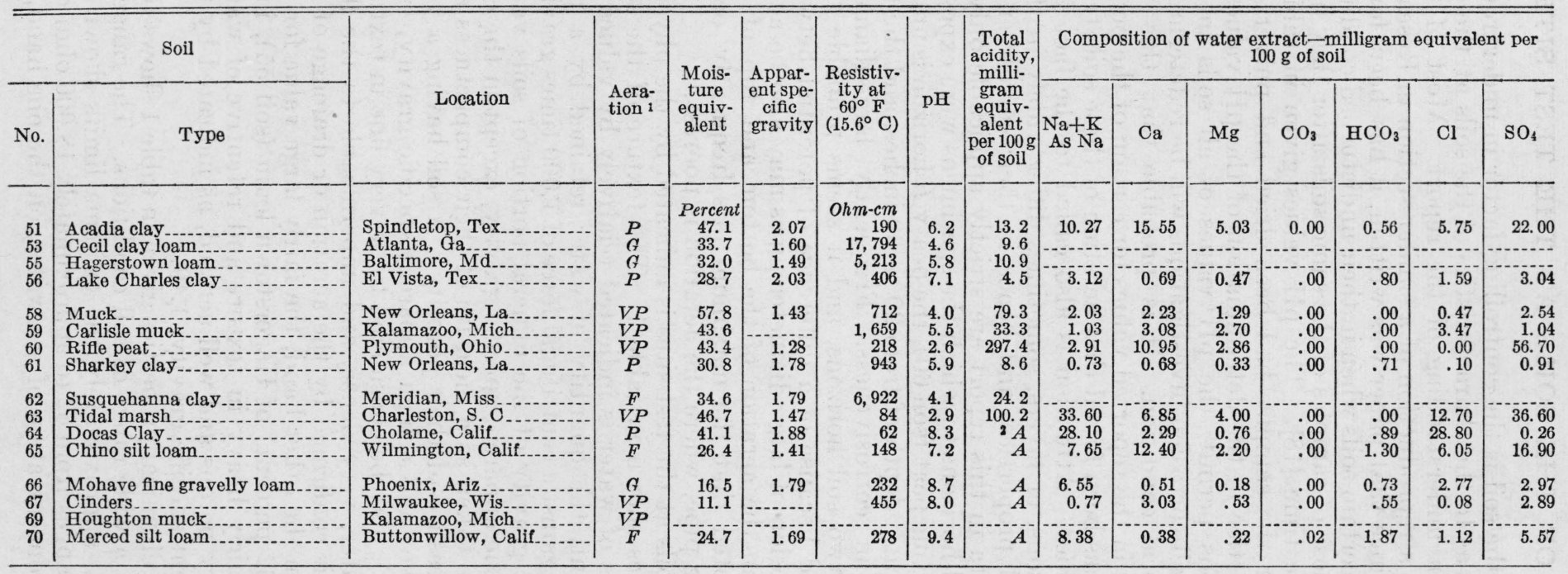

1 Aeration of soils: $G$, good; $F$, fair; $P$, poor; $V P$, very poor.

Alkaline. 
the concentration of salts in a highly weathered soil, on the other. The soluble material in Rifle peat (soil 60), consists almost exclusively of sulfates. This soil is extremely acid, so much so that the soil actually contains sulfuric acid. In Docas clay (soil 64) the soluble material is almot entirely sodium chloride.

The names of the soils given in table 1 were assigned by the Soil Survey of the Bureau of Plant Industry of the United States Department of Agriculture. That part of the name which describes the texture of the soil refers to the texture of the uppermost, or $A$, horizon. As the specimens were buried at depths from 18 inches to 4 feet, they usually lie in the $B$ or $C$ horizon. As these horizons are frequently heavier in texture than the $A$ horizon, the aeration of the soil in which the specimen lies may be poorer than is suggested by the soil types.

\section{FERROUS MATERIALS}

\section{CAST MATERIALS}

\section{(a) DIMENSIONS AND COMPOSITION}

Table 2 shows the composition and dimensions of the cast materials. The materials $\mathrm{CB}$ and $\mathrm{CC}$ were parts of a composite specimen consisting of a piece of deLavaud cast iron bolted to a piece of charcoal cast iron by means of a charcoal cast-iron bolt (CD) and a steel bolt (CE).

TABLE 2.-Dimensions and composition of cast materials

\begin{tabular}{|c|c|c|c|c|c|c|c|c|c|c|}
\hline Material & & \multicolumn{2}{|c|}{$\begin{array}{l}\text { Identifi- } \\
\text { cation }\end{array}$} & $\begin{array}{c}\text { Year } \\
\text { buried }\end{array}$ & \multicolumn{2}{|c|}{ Form } & $\begin{array}{l}\text { Nominal } \\
\text { width or } \\
\text { diameter }\end{array}$ & Length & \multicolumn{2}{|r|}{$\begin{array}{c}\text { Thick- } \\
\text { ness }\end{array}$} \\
\hline $\begin{array}{l}\text { Charcoal } 1 \text { cast iron } \\
\text { deLavaud } 12 \text { cast iron. } \\
\text { Rattled }{ }^{3} \text { cast iron } \\
\text { Sand-coated cast iron. }\end{array}$ & 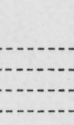 & \multicolumn{2}{|c|}{$\begin{array}{l}\text { CB } \\
\text { CC } \\
\text { G } \\
\text { F }\end{array}$} & $\begin{array}{l}1939 \\
1939 \\
1932 \\
1932\end{array}$ & \multicolumn{2}{|c|}{$\begin{array}{r}\text { Plate } \\
\text { Pipe...... } \\
\text { do do }\end{array}$} & $\begin{array}{l}\text { in. } \\
\text { 3.5 } \\
3.5 \\
1.25 \\
1.25\end{array}$ & \multicolumn{2}{|c|}{$\begin{array}{l}\text { in. } \\
12 \\
12 \\
13.5 \\
13.5\end{array}$} & $\begin{array}{l}\text { in. } \\
0.375 \\
.50 \\
.250 \\
.250\end{array}$ \\
\hline $\begin{array}{l}\text { Low-alloy cast iron } \\
\text { Do } \\
\text { Do } \\
\text { High-alloy cast iron }\end{array}$ & & \multicolumn{2}{|c|}{$\begin{array}{l}\mathrm{I} \\
\mathrm{J} \\
\mathrm{C} \\
\mathrm{E}\end{array}$} & $\begin{array}{l}1932 \\
1932 \\
1932 \\
1932\end{array}$ & \multicolumn{2}{|c|}{ 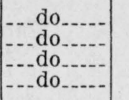 } & $\begin{array}{l}1.25 \\
1.25 \\
1.5 \\
1.5\end{array}$ & \multicolumn{2}{|l|}{$\begin{array}{l}12 \\
12 \\
12 \\
10\end{array}$} & $\begin{array}{l}.350 \\
.350 \\
.250 \\
.250\end{array}$ \\
\hline \multirow[b]{2}{*}{ Material } & \multicolumn{3}{|c|}{ C } & \multirow[b]{2}{*}{$\mathrm{Si}$} & \multirow[b]{2}{*}{ Mn } & \multirow[b]{2}{*}{$\mathrm{S}$} & \multirow[b]{2}{*}{$\mathrm{P}$} & \multirow[b]{2}{*}{$\mathrm{Cr}$} & \multirow[b]{2}{*}{$\mathrm{Ni}$} & \multirow[b]{2}{*}{$\mathrm{Cu}$} \\
\hline & Free & $\begin{array}{l}\text { Com- } \\
\text { bined }\end{array}$ & Total & & & & & & & \\
\hline 12 cast iron. & $\%$ & $\%$ & $\begin{array}{c}\% \\
2.40\end{array}$ & \multirow{6}{*}{$\begin{array}{l}\% \\
0.95 \\
1.51 \\
1.64 \\
1.64 \\
1.43 \\
2.04 \\
2.50 \\
2.13\end{array}$} & \multirow{6}{*}{$\begin{array}{c}\% \\
0.95 \\
.38 \\
.48 \\
.48 \\
.28 \\
.83 \\
.70 \\
1.00\end{array}$} & \multirow{6}{*}{$\begin{array}{c}\% \\
0.065 \\
.071 \\
.074 \\
.074 \\
.077 \\
.060 \\
.050\end{array}$} & \multirow{6}{*}{$\begin{array}{c}\% \\
0.17 \\
.78 \\
.79 \\
.79 \\
.128 \\
.248 \\
.400\end{array}$} & \multicolumn{2}{|r|}{$\%$} & $\begin{array}{l}\% \\
1.00\end{array}$ \\
\hline $\begin{array}{l}\text { deLavaud }{ }^{1}{ }^{2} \text { cast iron } \\
\text { Rattled }{ }^{3} \text { cast iron }\end{array}$ & & $\begin{array}{l}3.70 \\
0.64\end{array}$ & & & & & & $-\cdots-\cdot \mid-$ & -...- & $-\cdots$ \\
\hline Sgpd-coated cast iron & $\begin{array}{l}2.94 \\
2.94\end{array}$ & $\begin{array}{r}0.04 \\
.64\end{array}$ & $\begin{array}{l}3.5 \\
3.5\end{array}$ & & & & & $\mid-$ & -1 & 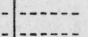 \\
\hline Low-alloy cast iron & $-\ldots$ & & & & & & & & -...- & .51 \\
\hline Do Do & 3.00 & & 2. 90 & & & & & & $-1=$ & .62 \\
\hline High-alloy cast iron & & & $\begin{array}{l}3.50 \\
2.98\end{array}$ & & & & & $\begin{array}{l}0.30 \\
2.61\end{array}$ & $\begin{array}{r}0.15 \\
15.00\end{array}$ & 6.58 \\
\hline
\end{tabular}

1 The deLavaud and charcoal cast-iron plates were connected by means of a charcoal cast-iron bolt (CD) and a steel bolt (CE).

2 Curved plate cut from 12-in. class 150 Super deLavaud pipe.

3 Ordinary iron horizontally cast in green sand molds and rattled to remove sand. 


\section{(b) SPECIMENS EXPOSED FOR 9 YEARS}

Tables 3 and 4 show the losses of weight and the depths of maximum pits for the cast specimens exposed approximately 9 years. The maximum pit depth recorded in table 4 is the average of the deepest pit on each of the two specimens of $1 \frac{11}{4}$-inch pipe about 1 foot long. It will be noted that in approximately 10 percent of the cases in table 3 and in 30 percent of the cases in table 4 , the average loss of weight or the average depth of the maximum pits was less for the 9 -year-old specimens than for the corresponding 7-year-old ones. In most of these cases the single maximum value was also greater for the earlier removals. This lack of reproducibility of the data is characteristic of underground corrosion and may be attributed to lack of homogeneity in the material under test, in the soil, or in the conditions of the test. In other words, the specimens examined were not sufficiently large to constitute adequate or representative samples, especially with respect to maximum pit depth. Studies of the relation between the maximum pit depth and the magnitude of the area from which the maximum pit depth was selected [14] indicate that the area would have to be very large before a deeper pit could not be expected to occur on a larger area. Because of this characteristic of the data on maximum pit depths, comparison of the depths of maximum pits on individual specimens should be made with caution.

TABLE 3.-Loss of weight of cast-iron pipe exposed for 9 years

[In ounces per square foot a]

\begin{tabular}{|c|c|c|c|c|c|c|c|c|c|}
\hline \multirow[b]{3}{*}{ No. } & Soil & \multirow{3}{*}{$\begin{array}{l}\text { Expo- } \\
\text { sure }\end{array}$} & \multirow{3}{*}{$\begin{array}{c}\text { Hori- } \\
\text { zontally } \\
\text { cast in } \\
\text { sand } \\
\text { mold, } \\
G\end{array}$} & \multicolumn{5}{|c|}{ Low-alloy } & \multirow{3}{*}{$\begin{array}{l}\text { High- } \\
\text { alloy } \\
\qquad E\end{array}$} \\
\hline & \multirow[b]{2}{*}{ Type } & & & \multirow[b]{2}{*}{$I$} & \multirow[b]{2}{*}{$J$} & \multicolumn{2}{|c|}{$I+J$} & \multirow[b]{2}{*}{$C$} & \\
\hline & & & & & & $\begin{array}{l}\text { Aver- } \\
\text { age }\end{array}$ & $\begin{array}{l}\text { Stand- } \\
\text { ard } \\
\text { error }\end{array}$ & & \\
\hline 53 & Cecil clay loam & $\begin{array}{r}\text { Years } \\
9.47\end{array}$ & 3.25 & & & & & & \\
\hline 55 & Hagerstown loam.- & 9.11 & $\begin{array}{l}5.20 \\
3.55\end{array}$ & 2.67 & b 2.10 & $\begin{array}{l}2.00 \\
2.38\end{array}$ & 0.2 & 2.04 & $\begin{aligned} & 1.00 \\
& \circ 0.72\end{aligned}$ \\
\hline 56 & Lake Charies clay. & 9.42 & d $D(42)$ & 37.34 & 33. 71 & 35.52 & 3.0 & 29.70 & 14.62 \\
\hline 58 & Muck & 9.51 & 20.08 & 24.05 & 27.10 & 25.58 & 1.0 & 21.88 & 9.91 \\
\hline 59 & Carlisle muck & 9.12 & b e 3.60 & 4.08 & b 2.92 & 3.50 & 0.4 & e 2.37 & 0.66 \\
\hline 60 & Rifle peat & 9.24 & 20.77 & - 18.15 & - 17.01 & 17.58 & 4.2 & e 13.07 & - 10.00 \\
\hline 61 & Sharkey clay..... & 9.53 & 7.08 & 7.54 & 6.89 & 7.22 & 0.4 & 7.21 & 2. 33 \\
\hline 62 & Susquehanna clay & 9.47 & 8.10 & 6.61 & 6.37 & 6.49 & .9 & 6.92 & 2. 73 \\
\hline 63 & Tidal marsh....... & 9.55 & 6.14 & - 9.98 & e 11.40 & 10.69 & 3.5 & 2.56 & 1.63 \\
\hline 64 & Docas clay & 9.21 & $D(34)$ & 46.83 & 44.59 & 45.71 & 0.8 & bf 41.93 & 12.82 \\
\hline 65 & Chino silt loam & 9.25 & 7.62 & 11.04 & 10.28 & 10.66 & .8 & 14.61 & e 2.55 \\
\hline 66 & Mohave fine gravelly loam..... & 9.23 & b 3.96 & 8.22 & 12.15 & 10.18 & 1.3 & 10.74 & $\circ 3.29$ \\
\hline 67 & Cinders............. & 9.24 & $D$ & $\mathbf{g} 61.41+$ & s64.99+ & 63.20 & & 45.74 & 52.33 \\
\hline
\end{tabular}

a Each ounce per square foot corresponds to an average penetration of 0.0017 inch.

b A verage loss of weight of 1939 removals is greater. The maximum loss of weight of the individual specimens of the 1939 removals is greater.

c A verage loss of weight of 1939 removals is greater. The maximum loss of weight of the individual speejmens of the 1941 removals is greater.

$\mathrm{d} D$, both specimens destroyed. The number in parentheses is the approximate loss of weight.

i Loss of weight of individual specimens differed from each other by more than 50 percent.

f Data for 1 specimen only.

g Data for 1 specimen only. The other specimen was destroyed by corrosion. 
TABLE 4.-Depths of maximum penetration on cast-iron pipe exposed for 9 years

[In mils]

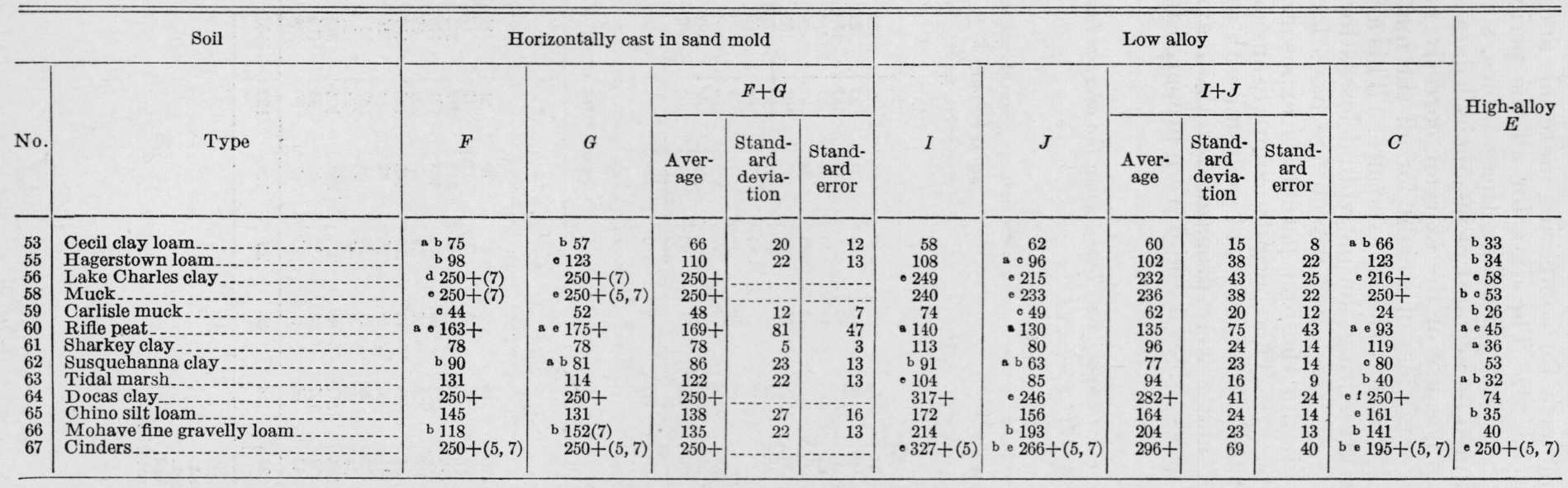

a Maximum pit depths of individual specimens differed from each other by more than 50 percent.

A verabe pit depths of the 1939 removals are greater. The single maximum pit depths of the 1939 removals are greater.

The plus $(+)$ sign indicates that 1 or both specimens were punctured. A number in parentheses after the pit depth indicates that 1 or both specimens of a previous removal

was punctured, e. g., (2) indicates a puncture after 2 years, etc.

Data for 1 specimen only. 


\section{Journal of Research of the National Bureau of Standards}

To provide a more reliable basis for comparing materials and soils, tables 5 to 8 have been prepared. The data for all the periods of exposure from 12 soils were used in compiling these tables, soils 51, 57, and 67 having been excluded on account of insufficient data or the destruction of the specimens because of the extreme corrosiveness of the soils. In table 5 the average loss of weight for all the materials in all the soils was obtained for each period of exposure. This average was used as a reference for presenting the individual losses for each material in each of the soils on a relative percentage basis for that particular period. The results for the four periods of exposure were then averaged to form table 6 . This permits comparisons of the materials in each soil and of the corrosiveness of each soil with respect to each material. Tables 7 and 8 were prepared for the maximum penetrations in the same way as tables 5 and 6 were prepared for the loss of weights.

TABLE 5.-Relative loss of weight of cast specimens based upon the average loss of all specimens for each period

[In percent]

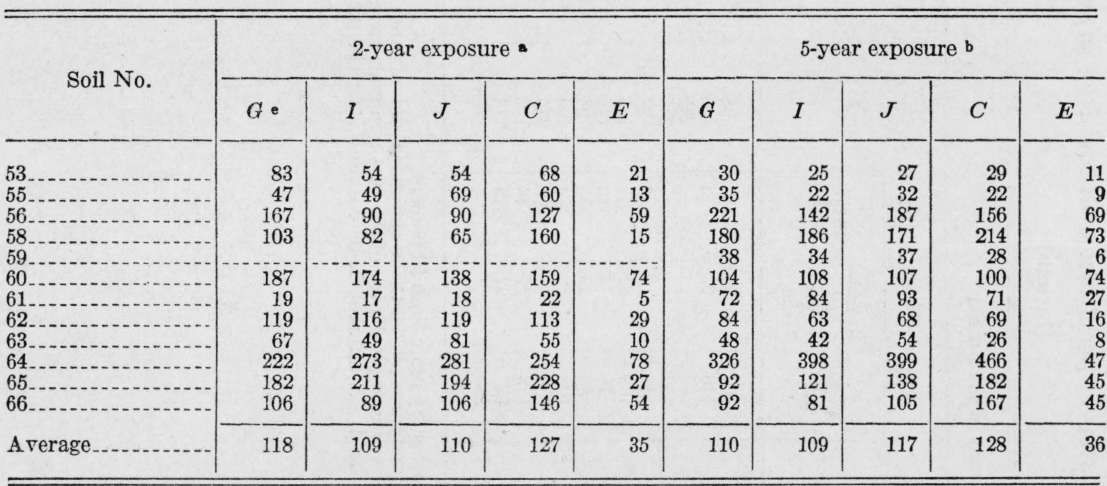

\begin{tabular}{|c|c|c|c|c|c|c|c|c|c|c|}
\hline \multirow{2}{*}{ Soil No. } & \multicolumn{5}{|c|}{ 7-year exposure 0} & \multicolumn{5}{|c|}{ 9-year exposure d } \\
\hline & $G$ & $I$ & $J$ & $C$ & $E$ & $G$ & $I$ & $J$ & $C$ & $E$ \\
\hline 60 & $\begin{array}{r}29 \\
35 \\
252 \\
217 \\
44 \\
56 \\
52 \\
57 \\
16 \\
406 \\
82 \\
64\end{array}$ & $\begin{array}{r}19 \\
27 \\
283 \\
236 \\
34 \\
83 \\
61 \\
49 \\
40 \\
451 \\
92 \\
54\end{array}$ & $\begin{array}{r}20 \\
28 \\
260 \\
229 \\
37 \\
65 \\
61 \\
55 \\
34 \\
505 \\
103 \\
87\end{array}$ & $\begin{array}{r}19 \\
23 \\
224 \\
206 \\
25 \\
49 \\
57 \\
28 \\
24 \\
512 \\
127 \\
69\end{array}$ & $\begin{array}{r}8 \\
8 \\
108 \\
99 \\
7 \\
15 \\
19 \\
11 \\
8 \\
66 \\
23 \\
41\end{array}$ & $\begin{array}{r}27 \\
29 \\
343 \\
164 \\
29 \\
170 \\
58 \\
66 \\
50 \\
278 \\
62 \\
32\end{array}$ & $\begin{array}{r}22 \\
22 \\
305 \\
196 \\
33 \\
148 \\
62 \\
54 \\
82 \\
383 \\
90 \\
67\end{array}$ & $\begin{array}{r}21 \\
17 \\
275 \\
221 \\
24 \\
139 \\
56 \\
52 \\
93 \\
364 \\
84 \\
99\end{array}$ & $\begin{array}{r}18 \\
17 \\
242 \\
179 \\
19 \\
107 \\
59 \\
57 \\
21 \\
343 \\
119 \\
88\end{array}$ & $\begin{array}{r}11 \\
6 \\
119 \\
81 \\
54 \\
82 \\
19 \\
22 \\
13 \\
105 \\
21 \\
27\end{array}$ \\
\hline A verage & 109 & 119 & 124 & 114 & 34 & 109 & 122 & 120 & 106 & 47 \\
\hline
\end{tabular}

a Average loss $=5.66$ ounces per square foot.

b A verage loss $=6.69$ ounces per square foot.

- A verage loss =8.73 ounces per square foot.

d A verage loss $=12.24$ ounces per square foot.

- See table 2 for the composition of the materials. 
TABLE 6.-Average of the relative loss of weight of the cast materials for four periods of exposure.

[In percent]

\begin{tabular}{|c|c|c|c|c|c|c|c|c|c|c|c|}
\hline \multirow[b]{2}{*}{ Soil No. } & \multicolumn{2}{|c|}{$G$ : } & \multicolumn{2}{|c|}{$I$} & \multicolumn{2}{|c|}{$J$} & \multicolumn{2}{|c|}{$C$} & \multicolumn{2}{|c|}{$E$} & \multirow[b]{2}{*}{$\begin{array}{c}\text { Aver- } \\
\text { age }\end{array}$} \\
\hline & $\begin{array}{l}\text { Rela- } \\
\text { tive } \\
\text { loss }\end{array}$ & $\begin{array}{l}\text { Stand- } \\
\text { ard } \\
\text { error }\end{array}$ & $\begin{array}{l}\text { Rela- } \\
\text { tive } \\
\text { loss }\end{array}$ & $\begin{array}{l}\text { Stand- } \\
\text { ard } \\
\text { error }\end{array}$ & $\begin{array}{l}\text { Rela- } \\
\text { tive } \\
\text { loss }\end{array}$ & $\begin{array}{l}\text { Stand- } \\
\text { ard } \\
\text { error }\end{array}$ & $\begin{array}{l}\text { Rela- } \\
\text { tive } \\
\text { loss }\end{array}$ & $\begin{array}{l}\text { Stand- } \\
\text { ard } \\
\text { error }\end{array}$ & $\begin{array}{l}\text { Rela- } \\
\text { tive } \\
\text { loss }\end{array}$ & $\begin{array}{l}\text { Stand- } \\
\text { ard } \\
\text { error }\end{array}$ & \\
\hline $\begin{array}{l}53 \\
556 \\
58 \\
59 \mathrm{~b} \\
60 \\
61 \\
62 \\
645 \\
66\end{array}$ & $\begin{array}{r}42 \\
36 \\
246 \\
166 \\
37 \\
129 \\
50 \\
82 \\
45 \\
308 \\
104 \\
74\end{array}$ & $\begin{array}{r}14 \\
5 \\
36 \\
24 \\
4 \\
31 \\
12 \\
13 \\
11 \\
39 \\
27 \\
16\end{array}$ & $\begin{array}{r}30 \\
30 \\
205 \\
175 \\
34 \\
128 \\
56 \\
70 \\
53 \\
376 \\
128 \\
73\end{array}$ & $\begin{array}{r}8 \\
6 \\
53 \\
33 \\
0 \\
21 \\
14 \\
16 \\
10 \\
38 \\
29 \\
7\end{array}$ & $\begin{array}{r}30 \\
34 \\
203 \\
172 \\
33 \\
112 \\
57 \\
74 \\
66 \\
387 \\
130 \\
99\end{array}$ & $\begin{array}{r}9 \\
14 \\
42 \\
37 \\
3 \\
18 \\
15 \\
15 \\
12 \\
47 \\
24 \\
6\end{array}$ & $\begin{array}{r}34 \\
30 \\
187 \\
190 \\
24 \\
104 \\
52 \\
67 \\
32 \\
394 \\
164 \\
118\end{array}$ & $\begin{array}{r}11 \\
10 \\
28 \\
11 \\
3 \\
22 \\
11 \\
17 \\
7 \\
58 \\
26 \\
22\end{array}$ & $\begin{array}{r}13 \\
9 \\
89 \\
67 \\
22 \\
61 \\
18 \\
20 \\
10 \\
74 \\
29 \\
42\end{array}$ & $\begin{array}{r}2 \\
2 \\
14 \\
18 \\
16 \\
16 \\
4 \\
3 \\
1 \\
12 \\
6 \\
5\end{array}$ & $\begin{array}{r}30 \\
28 \\
186 \\
154 \\
25 \\
107 \\
47 \\
63 \\
41 \\
256 \\
111 \\
81\end{array}$ \\
\hline A verage... & 110 & 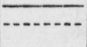 & 113 & $-\ldots \ldots$ & $\overline{116}$ & $\overline{c . . . . . . . .}$ & 116 & - & 38 & ann & $\cdots$ \\
\hline
\end{tabular}

a See table 2 for composition of the materials.

b Data for only 3 periods of exposure.

TABLE 7.-Relative maximum penetration of cast specimens based upon the average maximum penetration of all specimens for each period

[In percent]

\begin{tabular}{|c|c|c|c|c|c|c|c|c|c|c|c|c|}
\hline \multirow{2}{*}{ Soil No. } & \multicolumn{6}{|c|}{ 2-year exposure } & \multicolumn{6}{|c|}{ 5-year exposure b } \\
\hline & $F \bullet$ & $G$ & $I$ & $J$ & $C$ & $E$ & $F$ & $G$ & $I$ & $J$ & $C$ & $E$ \\
\hline $\begin{array}{l}53 \\
55 \\
56 \\
58 \\
59 \\
60 \\
61 \\
62 \\
63 \\
65\end{array}$ & $\begin{array}{r}104 \\
50 \\
145 \\
79 \\
272 \\
95 \\
116\end{array}$ & $\begin{array}{r}108 \\
89 \\
133 \\
50 \\
257 \\
104 \\
124\end{array}$ & $\begin{array}{r}62 \\
58 \\
46 \\
85 \\
79 \\
62 \\
207 \\
41 \\
282 \\
116 \\
118\end{array}$ & $\begin{array}{r}79 \\
54 \\
141 \\
106 \\
249 \\
120 \\
112\end{array}$ & $\begin{array}{r}81 \\
54 \\
66 \\
91 \\
71 \\
31 \\
166 \\
21 \\
295 \\
120 \\
141\end{array}$ & $\begin{array}{l}50 \\
75 \\
89 \\
46 \\
58 \\
54 \\
54\end{array}$ & $\begin{array}{r}71 \\
95 \\
171 \\
150 \\
75 \\
86 \\
69 \\
95 \\
62 \\
167 \\
107 \\
102\end{array}$ & $\begin{array}{r}73 \\
72 \\
157 \\
258 \\
48 \\
98 \\
79 \\
137 \\
107 \\
181 \\
135 \\
114\end{array}$ & $\begin{array}{r}68 \\
81 \\
141 \\
154 \\
62 \\
72 \\
120 \\
102 \\
86 \\
150 \\
137 \\
86\end{array}$ & $\begin{array}{r}65 \\
85 \\
134 \\
157 \\
50 \\
82 \\
86 \\
115 \\
120 \\
150 \\
144 \\
101\end{array}$ & $\begin{array}{r}58 \\
83 \\
145 \\
179 \\
16 \\
104 \\
63 \\
109 \\
17 \\
210 \\
131 \\
173\end{array}$ & $\begin{array}{r}52 \\
53 \\
46 \\
49 \\
20 \\
115 \\
56 \\
71 \\
23 \\
39 \\
60 \\
52\end{array}$ \\
\hline A verage...... & 111 & 107 & 105 & 106 & 103 & 67 & 104 & 122 & 105 & 107 & 107 & 53 \\
\hline \multirow{2}{*}{ Soil No. } & \multicolumn{6}{|c|}{ 7-year exposure 0} & \multicolumn{6}{|c|}{9 -year exposure $d$} \\
\hline & $F$ & $G$ & $I$ & $J$ & $C$ & $E$ & $F$ & $G$ & $I$ & $J$ & $C$ & $E$ \\
\hline $\begin{array}{l}63 \\
64 \\
65\end{array}$ & $\begin{array}{r}103 \\
127 \\
252 \\
201 \\
46 \\
35 \\
56 \\
107 \\
52 \\
151 \\
87 \\
123\end{array}$ & $\begin{array}{r}71 \\
127 \\
252 \\
252 \\
52 \\
26 \\
76 \\
119 \\
61 \\
123 \\
113 \\
182\end{array}$ & $\begin{array}{r}50 \\
91 \\
192 \\
193 \\
44 \\
81 \\
91 \\
95 \\
91 \\
147 \\
111 \\
150\end{array}$ & $\begin{array}{r}60 \\
98 \\
185 \\
180 \\
57 \\
25 \\
78 \\
84 \\
72 \\
157 \\
119 \\
201\end{array}$ & $\begin{array}{r}89 \\
96 \\
151 \\
177 \\
20 \\
18 \\
53 \\
83 \\
133 \\
144 \\
129 \\
162\end{array}$ & $\begin{array}{l}51 \\
41 \\
53 \\
58 \\
28 \\
22 \\
30 \\
37 \\
55 \\
40 \\
42 \\
38\end{array}$ & $\begin{array}{r}60 \\
79 \\
201 \\
201 \\
35 \\
131 \\
63 \\
72 \\
105 \\
201 \\
116 \\
95\end{array}$ & $\begin{array}{r}46 \\
99 \\
201 \\
201 \\
42 \\
140 \\
63 \\
65 \\
91 \\
201 \\
105 \\
122\end{array}$ & $\begin{array}{r}47 \\
87 \\
200 \\
193 \\
59 \\
112 \\
91 \\
73 \\
83 \\
254 \\
138 \\
172\end{array}$ & $\begin{array}{r}50 \\
82 \\
173 \\
187 \\
39 \\
104 \\
64 \\
51 \\
68 \\
197 \\
125 \\
155\end{array}$ & $\begin{array}{r}53 \\
99 \\
173 \\
201 \\
19 \\
75 \\
96 \\
64 \\
32 \\
201 \\
129 \\
113\end{array}$ & $\begin{array}{r}26 \\
27 \\
47 \\
43 \\
21 \\
36 \\
29 \\
43 \\
26 \\
59 \\
128 \\
32\end{array}$ \\
\hline A verage..... & 112 & 121 & 104 & 110 & 104 & 41 & 113 & 115 & 126 & 108 & 104 & 85 \\
\hline
\end{tabular}

- A verage maximum pit depth $=48$ mils.

b A verage maximum pit depth $=69$ mils.

- Average maximum pit depth $=99$ mils.

d A verage maximum pit depth $=125$ mils.

- See table 2 for composition of the materials. 
TABLE 8.-Average of the relative maximum penetration of the cast materials for the four periods of exposure

[In percent]

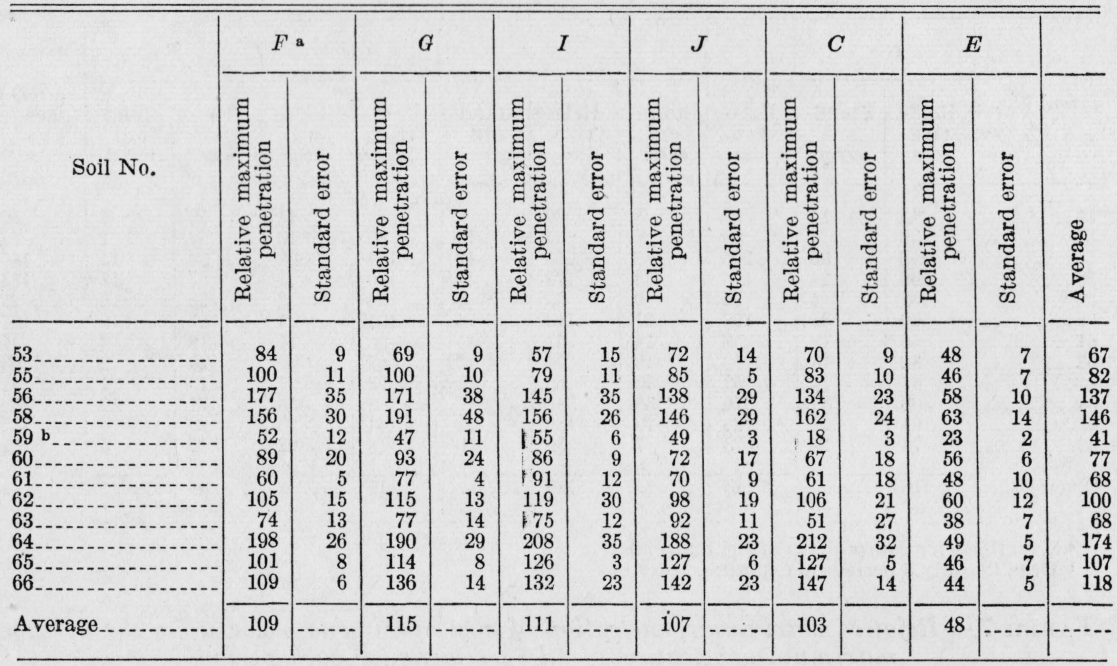

a See table 2 for the composition of the materials.

b Data for only 3 periods of exposure.

An analysis of the data in tables 5 and 7 indicates that the highalloy cast iron, $E$, is definitely superior with respect to loss of weight and pitting to the other cast irons in all the soils The data also show that, except in a very few cases, there are no real, consistent differences in the loss of weight or maximum penetration between any of the plain or low-alloy cast irons. It will be noted that for all the periods the low-alloy cast iron, $C$, is consistently better than the other low-alloy or plain cast iron in soil 59 with respect to loss of weight and maximum penetration; and that plain cast iron, $G$, is consistently better than the others in soil 64 and 65 with respect to loss of weight only. The summary of the relative values in tables 6 and 8 shows that with respect to loss of weight the composite averages of all periods for the plain or low-alloy cast irons agree within 6 percent, and with respect to pitting, the composite averages of all periods for the same materials agree within 12 percent It is doubtful that the small differences can be considered significant in view of the standard error of the averages. Hence, the data bring out the fact that none of the plain or low-alloy cast irons is definitely superior, in general, to the other materials in the 12 soils tested. Figure 1 shows the specimens of cast iron removed from Mohave fine gravelly loam (soil 66) after 9 years of exposure.

\section{(c) SPECIMENS EXPOSED FOR 2 YEARS}

The cast-iron specimens exposed for 2 years consist of 12 by $3 \frac{1}{2}$ by $1 / 2$-inch sections of 12 -inch class 150 Super deLavaud pipe bolted to flat 12 by $3 \frac{1}{2}$ by $3 / 8$-inch charcoal cast-iron plates by means of steel and charcoal cast-iron bolts.

Table 9 indicates that the charcoal cast iron lost more weight than the Super deLavaud iron in most soils, and in all but one soil the maximum pit depths on the Super deLavaud cast iron were definitely less 

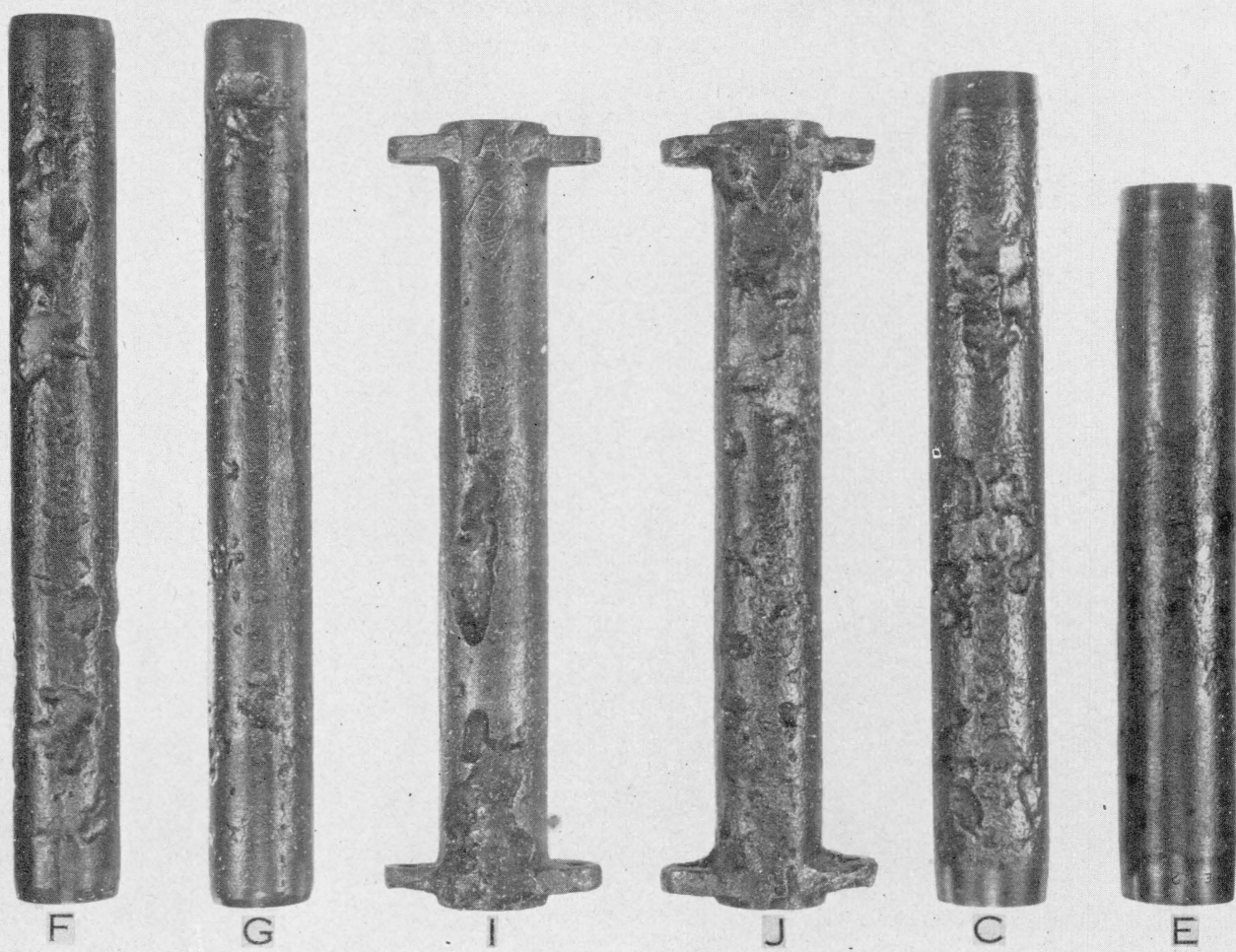

Figure 1.-Cast-iron specimens exposed to soil 66 for 9 years.

$F$, Sand-coated; $G$, rattled; $I$, low-alloy; $J$, low-alloy; $C$, low-alloy; $E$, high-alloy. 

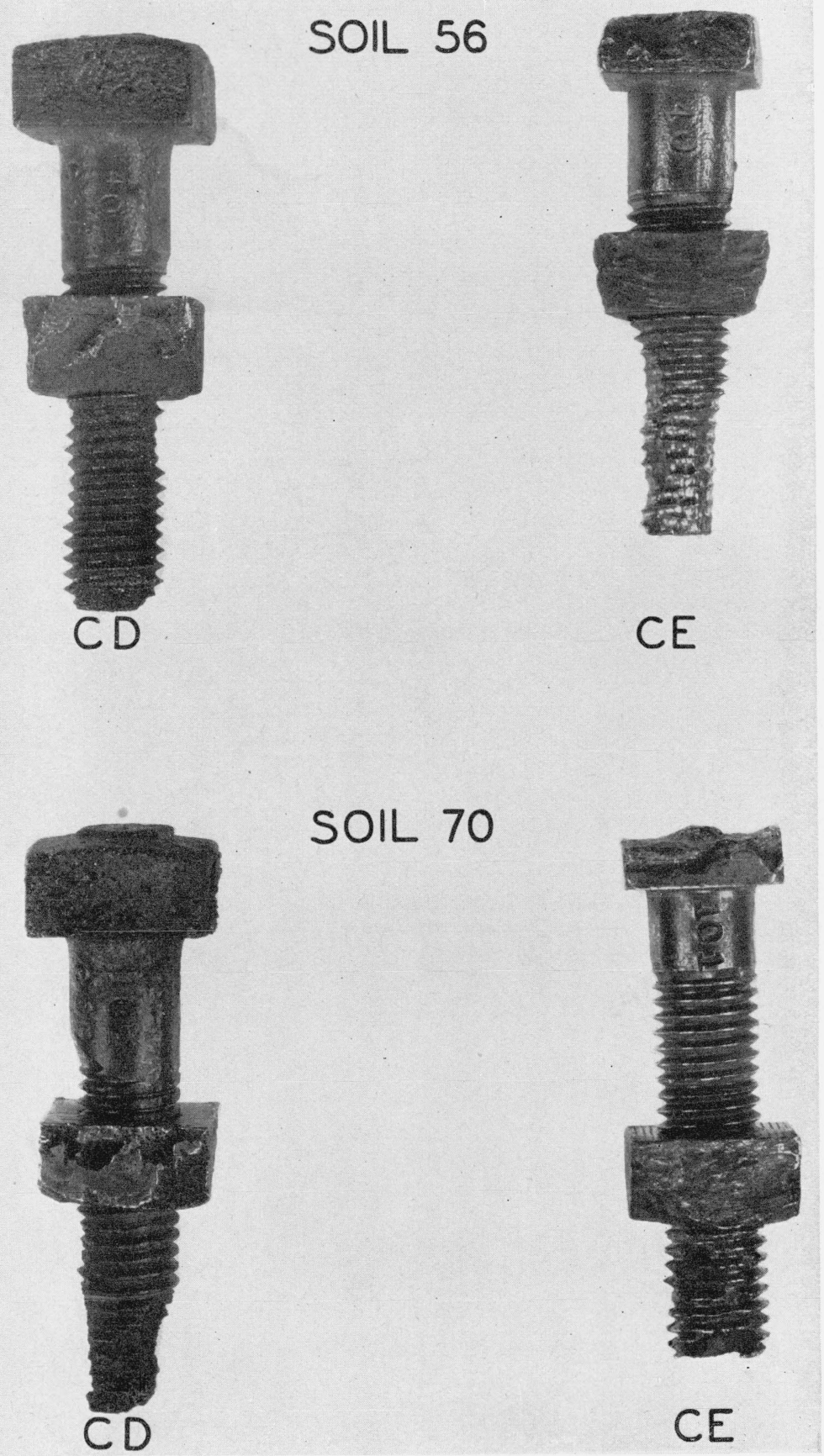

Figure 2.-Charcoal cast iron $(C D)$ and steel $(C E)$ bolts exposed to both soil 56 and 70 for approximately 2 years each. 
than on the charcoal cast iron. This might be attributed to galvanic action between the different metals in addition to the other causes of corrosion. Figure 2 shows the condition of the cast-iron and steel bolts which were connected to the couples in soils 56 and 70 .

TABLE 9.-Loss of weight and depth of maximum penetration of cast-iron plates and cast-iron and steel bolts (the plates were connected by the bolts)

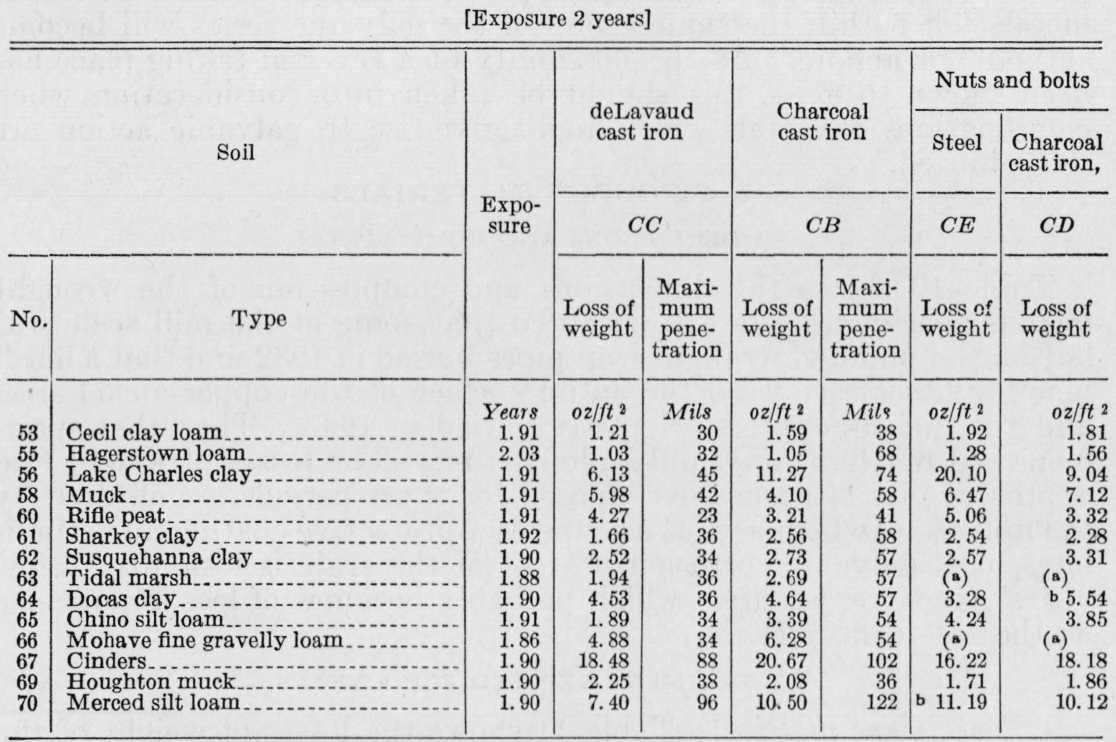

- Both specimens missing.

b Loss of weight for individual specimens differed from each other by more than 50 percent.

Laboratory measurements were made on the open-circuit differences of potential between a pair of the two different cast-iron plates buried in a box containing a saturated sample of Lake Charles clay (soil 56). The difference of potential reached the constant value of 61 millivolts after several weeks, the deLavaud specimen being cathodic. This is in accord with the data for soil 56 reported in table 9, which shows the loss of weight on the charcoal cast iron to be almost twice the loss on the Super deLavaud specimen. The pitting was also deeper on the charcoal cast iron. If it be assumed that the losses of weight due to the soil conditions alone were about the same for the two materials, the difference in corrosion observed in table 9 might be accounted for by the extra galvanic action caused by the dissimilarity between the two metals. As the charcoal cast iron was anodic, its tendency to corrode was greater. However, the deLavaud cast iron will not be cathodic in all soils, since the two types of cast iron would probably fall in the same group of the galvanic series set up by McKay and Worthington [19], who arranged the metals in groups according to their tendency to corrode galvanically, and showed that the relative positions of metals in the same group are subject to reversals. Such a reversal was observed on another set of measurements of a pair of the cast-iron materials in Docas clay (soil 64). The metals were buried in a very wet sample of the soil. The deLavaud cast iron was cathodic at first, but after considerable drying of the soil, a reversal 


\section{Journal of Research of the National Bureau of Standards}

took place and the Super deLavaud plate become anodic to the charcoal cast iron.

This shows that the environmental condition of the soil in which couples are buried plays an important role in corrosion due to galvanic action. The aeration of a soil is affected by its retentiveness of water, and since the aeration and water content vary from season to season, it cannot be forecast which part of a couple whose constituent metals fall within the same group of the galvanic series will become cathodic or anodic. As the possibility of a reversal taking place has been shown to exist, this should be taken into consideration when combinations of metals which might give rise to galvanic action are to be buried.

\section{WROUGHT MATERIALS}

\section{(a) DIMENSIONS AND COMPOSITION}

Table 10 shows the dimensions and composition of the wrought ferrous specimens. It will be noted that some of the mill scale was left on the puddled wrought-iron pipes buried in 1932 and that a hard, black mill scale covered the entire surface of the copper-nickel steel and the nickel-copper steel plates buried in 1937. The other specimens did not have any mill-scale coating. The losses of weight and depths of pits of specimens exposed for short periods are affected by the mill scale, which acts as an inperfect protective coating and stimulates local galvanic corrosion. Most of the scale is lost after a few years' exposure, an effect which probably becomes of less importance as the test continues.

\section{(b) SPECIMENS EXPOSED FOR 9 YEARS}

1. Specimens of Pipe.-Table 11 shows the losses of weight of the wrought ferrous pipe buried in 1932 and removed in 1941. In most soils the low-carbon steel, $N$, seems to lose a little more weight than most of the other materials, but usuially the differences are not great, except with respect to the specimens high in chromium.

Table 12 shows the averages of the deepest pits on the specimens of wrought pipe exposed 9 years. As was said in the discussion of the corresponding cast specimens, data on the maximum pit depths are too erratic to justify comparisons of materials unless a considerable number of specimens of each material is available. As an example, the data in table 12 show that corrosion had punctured the wall of seven of the pipes removed after 7 years of exposure, but that other specimens of the same materials were not punctured even after 9 years of exposure to the same soils. For the purpose of determining whether the data showed any real difference in loss of weight and in resistance to pitting for all periods of exposure, tables 13 to 16 were prepared according to the method used in constructing tables 5 to 8 . In addition to the soils omitted from those tables for the cast materials, soils 64 and 66 were excluded from tables 13 to 16 , since most of the specimens contained punctures in the last two removals. It was permissible to use these data for these two soils for the cast materials because of the greater wall thickness of the pipes. Tables 13 and 14 indicate that for all periods, with respect to loss of weight the 5-percent chromium steel $(P)$ is consistently better than any of the other specimens in soils 53 and 55 , and nickel-copper steel $(D)$ is consistently best in soil 65 . With respect to pitting, $D$ is consistently better than the other specimens in soil 53. 
TABLE 10.-Composition of wrought ferrous materials

\begin{tabular}{|c|c|c|c|c|c|c|c|c|c|c|c|c|c|c|c|c|}
\hline Material & $\mid \begin{array}{c}\text { Iden- } \\
\text { tifica- } \\
\text { tion }\end{array}$ & $\begin{array}{c}\text { Year } \\
\text { buried }\end{array}$ & Form & $\begin{array}{l}\text { Nominal } \\
\text { sidth or } \\
\text { diameter }\end{array}$ & Length & $\begin{array}{c}\text { Thick- } \\
\text { ness }\end{array}$ & $\mathrm{C}$ & $\mathrm{Si}$ & $\mathrm{Mn}$ & $\mathrm{S}$ & $\mathrm{P}$ & $\mathrm{Cr}$ & $\mathrm{Ni}$ & $\mathrm{Cu}$ & Mo & Other elements \\
\hline \multicolumn{17}{|c|}{ WROUGHT IRON } \\
\hline $\begin{array}{l}\text { Hand-puddled } \\
\text { Roe process. }\end{array}$ & $\begin{array}{l}A \mathrm{a} \\
B \mathrm{a}\end{array}$ & $\begin{array}{l}1932 \\
1932\end{array}$ & Pipe...- & $\begin{array}{l}\text { in. } \\
1.5 \\
1.5\end{array}$ & $\begin{array}{l}\text { in. } \\
12 \\
12\end{array}$ & $\begin{array}{l}\text { in. } \\
0.145 \\
.145\end{array}$ & $\begin{array}{l}\% \\
0.016 \\
.017\end{array}$ & $\begin{array}{l}\% \\
0.10 \\
.125\end{array}$ & $\begin{array}{l}\% \\
0.029 \\
.041\end{array}$ & $\begin{array}{l}\% \\
0.018 \\
.018\end{array}$ & $\begin{array}{c}\% \\
0.160 \\
.106\end{array}$ & $\%$ & $\%$ & \begin{tabular}{c|}
$\%$ \\
\hdashline \\
\hdashline-1 \\
\end{tabular} & $\%$ & $\begin{array}{l}\% \\
\text { Oxide + slag, } 2.56 \\
\text { Oxide + slag, } 2.681\end{array}$ \\
\hline \multicolumn{17}{|c|}{ CARBON STEELS } \\
\hline Low-carbon steel... & $N$ & 1932 & Pipe.... & 2.3 & 10 & 0.145 & 0.15 & & 0.49 & 0.030 & 0.013 & & & & & \\
\hline \multicolumn{17}{|c|}{ LOW-ALLOY IRONS AND STEELS } \\
\hline Open-hearth steel & $A$ & 1937 & Plate_. & 2.5 & 12 & 0.188 & 0.033 & 0.002 & 0.029 & 0.017 & 0.006 & 0.049 & 0.034 & 0.052 & & \\
\hline $\begin{array}{l}\text { Copper - molybdenum open, hearth } \\
\text { iron. }\end{array}$ & $M M$ & 1939 & ... do & 2.5 & 12 & .250 & .04 & & .16 & .027 & .008 & .04 & .14 & .051 & 0.07 & $\begin{array}{l}\mathrm{O}_{2}, 0.015 ; \mathrm{N}_{2}, 0.008 \\
\quad \mathrm{Sn}, 0.002\end{array}$ \\
\hline Do & $O$ & 1937 & ...do & 2.5 & 12 & .243 & .03 & .003 &. $\mathrm{i} 6$ & .032 & .007 & .02 & .15 & .45 & .07 & \\
\hline & $\stackrel{N}{H}$ & $\begin{array}{l}1937 \\
1932\end{array}$ & Pipe & $\begin{array}{l}2.5 \\
1.5\end{array}$ & $\begin{array}{l}12 \\
12\end{array}$ & .250 & $\begin{array}{l}.06 \\
.04\end{array}$ & .001 & $\begin{array}{l}.098 \\
.32\end{array}$ & $\begin{array}{l}.029 \\
.027\end{array}$ & $\begin{array}{l}.069 \\
.016\end{array}$ & .02 & .14 & $\begin{array}{l}.54 \\
.52\end{array}$ & $\begin{array}{l}.13 \\
.15\end{array}$ & \\
\hline Copper-nickel steel & $J \mathrm{~b}$ & 1937 & Plate.--- & 2.5 & 12 & .265 & .06 & .047 & $\begin{array}{l}.32 \\
.49\end{array}$ & .025 & .095 & $-\cdots$ & .52 & .95 & .15 & \\
\hline Nickel-copper steel. & $B \mathrm{~b}$ & 1937 & Pino do & 2.5 & 12 & .248 & .07 & .14 & .44 & .022 & .010 & $-\ldots$ & 1.96 & 1.01 & $-\ldots$ & \\
\hline Chromium - silicon - copper - phos- & $\stackrel{D}{C}$ & $\begin{array}{l}1932 \\
1937\end{array}$ & Pipe...- & $\begin{array}{l}1.5 \\
2.5\end{array}$ & $\begin{array}{l}12 \\
12\end{array}$ & $\begin{array}{l}.145 \\
.188\end{array}$ & $\begin{array}{l}.14 \\
.075\end{array}$ & $\begin{array}{l}.19 \\
.84\end{array}$ & $\begin{array}{l}.21 \\
.20\end{array}$ & .018 & .124 & 1.02 & $\begin{array}{l}2.47 \\
.022\end{array}$ & $\begin{array}{l}1.08 \\
.428\end{array}$ & 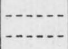 & \\
\hline $\begin{array}{l}\text { phorus steel. } \\
\text { 2\% cbromium steel with molybdenum } \\
\text { Do. }\end{array}$ & $\begin{array}{l}N N \\
K K\end{array}$ & $\begin{array}{l}1939 \\
1937\end{array}$ & $\begin{array}{l}\text { Pipe.-.- } \\
\text { Plate-.-- }\end{array}$ & $\begin{array}{l}1.5 \\
2.5\end{array}$ & ${ }_{12}^{14.5}$ & $\begin{array}{l}.145 \\
.175\end{array}$ & $\begin{array}{l}.09 \\
.082\end{array}$ & .25 & $\begin{array}{l}.46 \\
.46\end{array}$ & $\begin{array}{l}.010 \\
.015\end{array}$ & $\begin{array}{l}.015 \\
.017\end{array}$ & $\begin{array}{l}1.96 \\
2.01\end{array}$ & .07 & .004 & $\begin{array}{l}.49 \\
.57\end{array}$ & \\
\hline
\end{tabular}

a These specimens had some mill scale on the surface at time of burial.

b These specimens were completely covered with a hard, black mill scale at time of burial, 
TABLE 10.-Composition of wrought ferrous materials-Continued

\begin{tabular}{|c|c|c|c|c|c|c|c|c|c|c|c|c|c|c|c|c|}
\hline Material & $\begin{array}{l}\text { Iden- } \\
\text { tifica- } \\
\text { tion }\end{array}$ & Year & Form & $\begin{array}{l}\text { Nominal } \\
\text { width or } \\
\text { diameter }\end{array}$ & Length & $\begin{array}{c}\text { Thick- } \\
\text { ness }\end{array}$ & C & $\mathrm{Si}$ & Mn & $\mathrm{S}$ & $\mathbf{P}$ & $\mathrm{Cr}$ & $\mathrm{Ni}$ & $\mathrm{Cu}$ & Mo & Other elements \\
\hline \multicolumn{17}{|c|}{ CHROMIUM STEEL } \\
\hline $\begin{array}{l}4 \text { to } 6 \% \text { chromium steel } \\
\text { Do } \\
4 \text { to } 6 \% \text { chromium steel with } \\
\text { molybdenum. } \\
\text { Do. } \\
12 \% \text { chromium steel } \\
18 \% \text { chromium steel. } \\
\text { Do }\end{array}$ & $\begin{array}{l}P \\
D \\
E \\
H \\
U \\
V \\
X\end{array}$ & $\begin{array}{l}1932 \\
1937 \\
1937 \\
1937 \\
1932 \\
1932 \\
1932\end{array}$ & 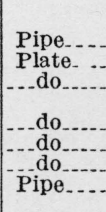 & $\begin{array}{ll}\text { in. } & \\
2.3 \\
2.5 \\
2.5 \\
\\
\\
2.5 \\
\quad 4 \\
\quad 4 \\
1.5\end{array}$ & $\begin{array}{r}\text { in } \\
10 \\
12 \\
12 \\
12 \\
6 \\
6 \\
12\end{array}$ & $\begin{array}{l}\text { in. } \\
0.154 \\
.245 \\
.188 \\
\\
.203 \\
.063 \\
.063 \\
.145\end{array}$ & $\begin{array}{c}\% \\
0.13 \\
.077 \\
.074 \\
\\
.060 \\
.065 \\
.070 \\
.12\end{array}$ & $\begin{array}{c}\% \\
0.43 \\
.41 \\
.39 \\
.28 \\
.34 \\
.277\end{array}$ & $\begin{array}{r}\% \\
0.46 \\
.37 \\
.32 \\
\\
.40 \\
.38 \\
.36 \\
.42\end{array}$ & $\begin{array}{c}\% \\
0.025 \\
.005 \\
.006 \\
.014 \\
.017 \\
.015 \\
.017\end{array}$ & $\begin{array}{c}\% \\
0.012 \\
.015 \\
.013 \\
.021 \\
.011 \\
.014 \\
.016\end{array}$ & $\begin{array}{r}\% \\
5.05 \\
5.02 \\
4.67 \\
5.76 \\
11.95 \\
17.08 \\
17.72\end{array}$ & $\begin{array}{c}\% \\
0.09 \\
.09 \\
.17 \\
.482 \\
.092 \\
.287\end{array}$ & $\begin{array}{c}\% \\
0.008 \\
.004 \\
.004 \\
.025 \\
.021 \\
\end{array}$ & $\begin{array}{c}\% \\
0.51 \\
.43 \\
\end{array}$ & $\begin{array}{l}\% \\
\mathrm{Al}, 0.030 ; \mathrm{Ti}, 0.022 \\
\mathrm{Al}, 0.27\end{array}$ \\
\hline \multicolumn{17}{|c|}{ HIGH-CHROMIUM NICKEL AND MANGANESE STEELS } \\
\hline $\begin{array}{l}18 \% \text { chromium steel with nickel. } \\
\text { Do... } \\
\text { Do... } \\
18 \% \text { chromium steel with nickel and } \\
\text { manganesg. } \\
\text { Do......... } \\
18 \% \text { chromium steel with nickel, man- } \\
\text { ganese, and molybdenum. } \\
22 \% \text { chromium steel with nickel and } \\
\text { manganese. } \\
22 \% \text { nickel-chromium steel with man- } \\
\text { ganese and molybdenum. }\end{array}$ & $\begin{array}{c}K \\
R \\
W \\
T \\
S \\
C M \\
Y \\
D T\end{array}$ & $\begin{array}{l}1932 \\
1932 \\
1932 \\
1932 \\
1932 \\
1939 \\
1932 \\
1939\end{array}$ & 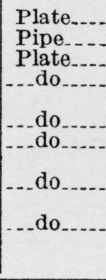 & $\begin{array}{l}3 \\
1.5 \\
4 \\
6 \\
6 \\
2 \\
4 \\
2.5\end{array}$ & $\begin{array}{c}11 \\
12 \\
6 \\
10 \\
10 \\
12.5 \\
6 \\
12\end{array}$ & $\begin{array}{l}0.025 \\
.145 \\
.063 \\
.063 \\
\\
.063 \\
.25 \\
.063 \\
.25\end{array}$ & $\begin{array}{l}0.08 \\
.05 \\
.093 \\
.06 \\
.07 \\
.07 \\
.144 \\
.07\end{array}$ & $\begin{array}{r}0.33 \\
.28 \\
.42 \\
.40 \\
.48 \\
.40 \\
.59 \\
.91\end{array}$ & $\begin{array}{r}0.44 \\
.46 \\
.36 \\
6.09 \\
9.44 \\
1.24 \\
1.80 \\
1.99\end{array}$ & $\begin{array}{l}.008 \\
.011 \\
.012\end{array}$ & $\begin{array}{l}.016 \\
.015 \\
.014\end{array}$ & $\begin{array}{r}17.20 \\
17.52 \\
18.69 \\
17.76 \\
17.78 \\
17.78 \\
22.68 \\
19.27\end{array}$ & $\begin{array}{l}10.96 \\
12.94 \\
22.12\end{array}$ & \begin{tabular}{c}
0.016 \\
.95 \\
.74 \\
\hdashline .021 \\
1.07
\end{tabular} & 3. 52 & 1 \\
\hline
\end{tabular}


TABLE 11.-Loss of weight of wrought pipe exposed for 9 years

[In ounces per square foot].

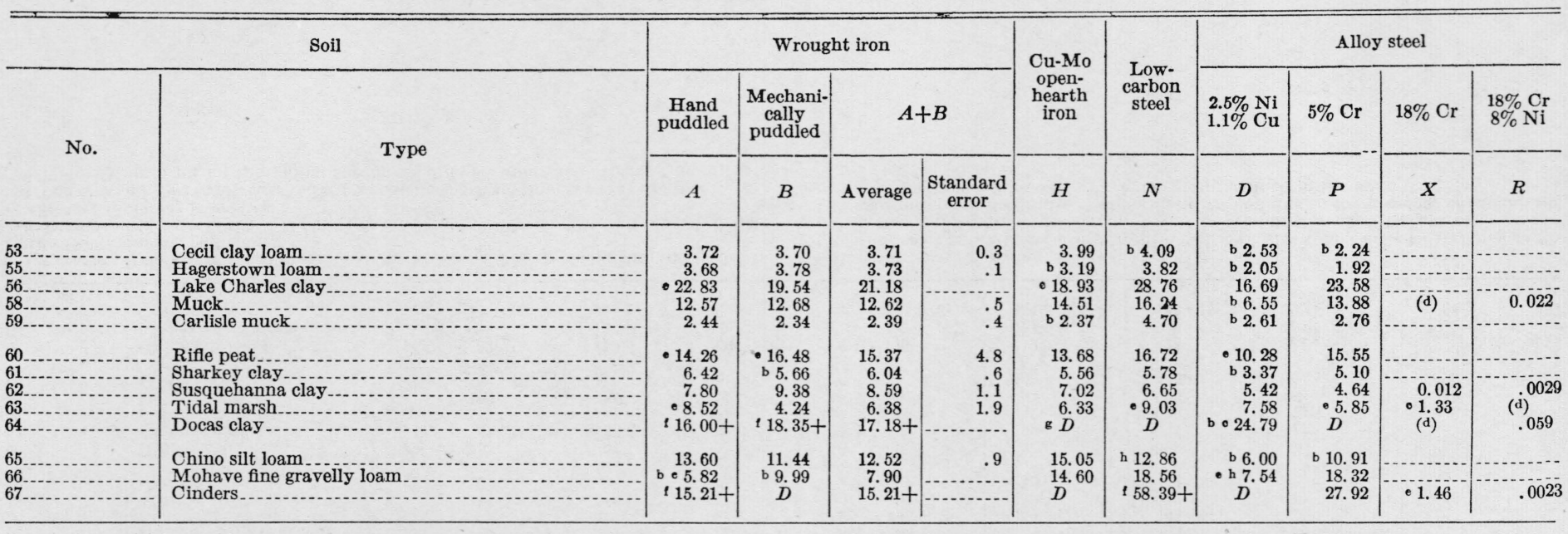

Aach ounce per square foot corresponds to an average penetration of 0.0015 inch

b Average loss of weight of 1939 removals is greater. The maximum loss of weight for the individual specimens of the 1939 removals is greater.

Data for 1 specimen only.

- Data for the individual specimens differed from the average by more than $50 \%$.

d Data cannot be used because of abnormal corrosion due to the presence of asphalt

g $D$, both specimens destroyed by corrosion.

h A verage loss of weight of 1939 removals is greater. The maximum loss of weight for

the individual specimens of the 1941 removals is greater. 
TABLE 12.-Depths of maximum pits on wrought pipe exposed for 9 years

[In mils]

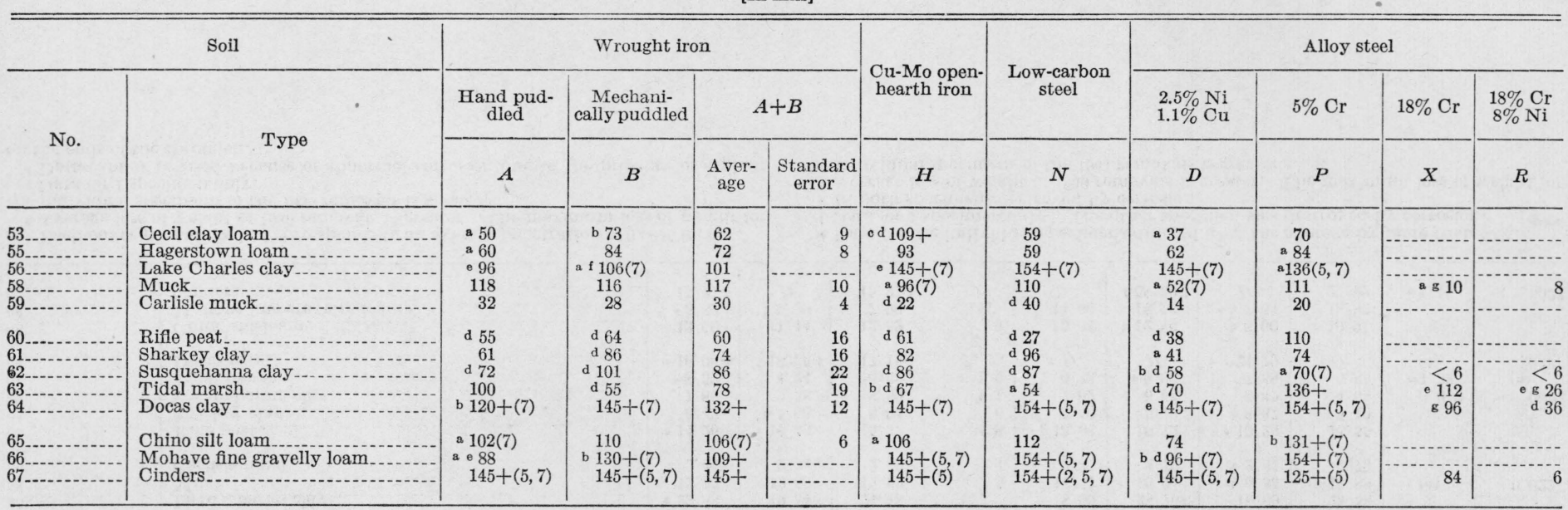

a Average pit depths of the 1939 removals are greater. The single maximum pit depth of the 1939 removals is greater.
$\mathrm{b}$ A verage pit depths of the 1939 removals are greater. The single maximum pit depth of the 1941 removals is greater.
The plus sign $(+)$ in all cases indicates that 1 or both specimens were punctured.

e Data for 1 specimen only.

1 A number in parentheses after the pit depth indicates that 1 or both specimens of a previous removal was punctured, e. g., (2) indicates a puncture after 2 years, etc.

$\mathrm{d}$ The maximum pit for individual specimens differed from each other by more than presence of asphalt on ends of the pipe. These pits have not been included in the data. 
TABLE 13.-Relative loss of weight of wrought specimens based upon the average loss of all specimens for each period

[In percent]

\begin{tabular}{|c|c|c|c|c|c|c|c|c|c|c|c|c|}
\hline \multirow{2}{*}{ Soil No. } & \multicolumn{6}{|c|}{ 2-year exposure $\mathrm{s}$} & \multicolumn{6}{|c|}{ 5-year exposure b } \\
\hline & $A^{\mathrm{e}}$ & $B$ & $H$ & $N$ & $D$ & $P$ & $A$ & $B$ & $H$ & $N$ & $D$ & $P$ \\
\hline $\begin{array}{l}53 \\
556 \\
56 \\
59 \\
59 \\
60 \\
61 \\
62 \\
63 \\
65\end{array}$ & \begin{tabular}{r}
104 \\
85 \\
105 \\
105 \\
\hdashline 170 \\
39 \\
90 \\
90 \\
238
\end{tabular} & $\begin{array}{r}150 \\
37 \\
120 \\
79 \\
184\end{array}$ & $\begin{array}{r}146 \\
29 \\
111 \\
75 \\
224\end{array}$ & $\begin{array}{r}187 \\
24 \\
122 \\
115 \\
222\end{array}$ & $\begin{array}{r}146 \\
35 \\
81 \\
47 \\
149\end{array}$ & $\begin{array}{r}129 \\
23 \\
72 \\
40 \\
216\end{array}$ & $\begin{array}{r}50 \\
44 \\
207 \\
187 \\
35 \\
121 \\
107 \\
77 \\
59 \\
142\end{array}$ & $\begin{array}{r}57 \\
45 \\
145 \\
199 \\
30 \\
131 \\
94 \\
76 \\
46 \\
139\end{array}$ & $\begin{array}{r}53 \\
35 \\
168 \\
199 \\
32 \\
111 \\
78 \\
79 \\
58 \\
214\end{array}$ & $\begin{array}{r}57 \\
42 \\
266 \\
213 \\
45 \\
210 \\
76 \\
90 \\
87 \\
196\end{array}$ & $\begin{array}{r}41 \\
26 \\
98 \\
147 \\
32 \\
150 \\
49 \\
44 \\
43 \\
80\end{array}$ & $\begin{array}{r}38 \\
23 \\
157 \\
142 \\
38 \\
223 \\
63 \\
50 \\
70 \\
186\end{array}$ \\
\hline Average..... & 114 & 110 & 98 & 115 & 86 & 76 & 103 & 96 & 103 & 128 & 71 & 99 \\
\hline \multirow{2}{*}{ Soil No. } & \multicolumn{6}{|c|}{ 7-year exposure 0} & \multicolumn{6}{|c|}{ 9-year exposure d } \\
\hline & $A$ & $B$ & $H$ & $N$ & $D$ & $P$ & $A$ & $B$ & $H$ & $N$ & $D$ & $P$ \\
\hline $\begin{array}{l}53 \\
55 \\
56 \\
59 \\
60 \\
61 \\
62 \\
65\end{array}$ & $\begin{array}{r}50 \\
52 \\
257 \\
179 \\
31 \\
77 \\
94 \\
90 \\
51 \\
136\end{array}$ & $\begin{array}{r}51 \\
50 \\
220 \\
174 \\
27 \\
81 \\
96 \\
90 \\
52 \\
132\end{array}$ & $\begin{array}{r}58 \\
51 \\
196 \\
184 \\
39 \\
67 \\
73 \\
76 \\
72 \\
221\end{array}$ & $\begin{array}{r}63 \\
48 \\
314 \\
211 \\
45 \\
114 \\
85 \\
80 \\
106 \\
206\end{array}$ & $\begin{array}{r}39 \\
32 \\
146 \\
146 \\
44 \\
57 \\
57 \\
55 \\
62 \\
91\end{array}$ & $\begin{array}{r}36 \\
25 \\
270 \\
176 \\
34 \\
43 \\
76 \\
52 \\
62 \\
201\end{array}$ & $\begin{array}{r}42 \\
41 \\
257 \\
141 \\
27 \\
160 \\
72 \\
87 \\
96 \\
153\end{array}$ & $\begin{array}{r}42 \\
42 \\
220 \\
143 \\
26 \\
185 \\
64 \\
105 \\
48 \\
129\end{array}$ & $\begin{array}{r}45 \\
36 \\
213 \\
163 \\
27 \\
154 \\
63 \\
79 \\
71 \\
169\end{array}$ & $\begin{array}{r}46 \\
43 \\
323 \\
183 \\
53 \\
188 \\
65 \\
75 \\
102 \\
145\end{array}$ & $\begin{array}{r}28 \\
23 \\
188 \\
74 \\
29 \\
116 \\
38 \\
61 \\
85 \\
67\end{array}$ & $\begin{array}{r}25 \\
22 \\
265 \\
156 \\
31 \\
175 \\
57 \\
52 \\
66 \\
123\end{array}$ \\
\hline A verage...- & 102 & 97 & 104 & 127 & 73 & 98 & 108 & 100 & 102 & 122 & 71 & 97 \\
\hline
\end{tabular}

A Average loss of weight $=3.34$ ounces per square foot.

b Average loss of weight $=5.23$ ounces pre square foot.

- A verage loss of weight $=6.67$ ounces per square foot.

d A verage loss of weight $=8.90$ ounces per square foot.

- See table 10 for the composition of the materiais.

TABLE 14.-Average of the relative loss of weights of wrought specimens for the four periods of exposure

[In percent]

\begin{tabular}{|c|c|c|c|c|c|c|c|c|c|c|c|c|c|}
\hline \multirow[b]{2}{*}{ Soil No. } & \multicolumn{2}{|c|}{$A^{\mathrm{s}}$} & \multicolumn{2}{|c|}{$B$} & \multicolumn{2}{|c|}{$H$} & \multicolumn{2}{|c|}{$N$} & \multicolumn{2}{|c|}{$D$} & \multicolumn{2}{|c|}{$P$} & \multirow[b]{2}{*}{ 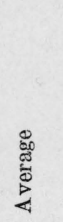 } \\
\hline & 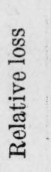 & 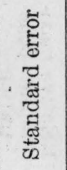 & 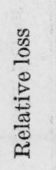 & 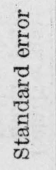 & 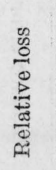 & 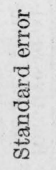 & 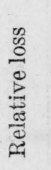 & 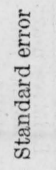 & 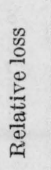 & 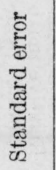 & 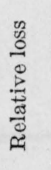 & 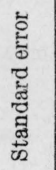 & \\
\hline $\begin{array}{l}53 \\
556 \\
58 \\
59 \mathrm{~b}- \\
601 \\
62 \\
63 \\
65\end{array}$ & $\begin{array}{r}62 \\
56 \\
206 \\
153 \\
31 \\
132 \\
78 \\
86 \\
74 \\
167\end{array}$ & $\begin{array}{r}14 \\
9 \\
37 \\
19 \\
2 \\
21 \\
15 \\
3 \\
11 \\
26\end{array}$ & $\begin{array}{r}63 \\
56 \\
179 \\
153 \\
28 \\
137 \\
73 \\
98 \\
56 \\
146\end{array}$ & $\begin{array}{r}14 \\
10 \\
24 \\
22 \\
1 \\
21 \\
14 \\
9 \\
8 \\
13\end{array}$ & $\begin{array}{r}60 \\
45 \\
162 \\
158 \\
33 \\
120 \\
61 \\
86 \\
69 \\
207\end{array}$ & $\begin{array}{r}8 \\
6 \\
31 \\
25 \\
1 \\
19 \\
11 \\
9 \\
4 \\
14\end{array}$ & $\begin{array}{r}62 \\
51 \\
256 \\
176 \\
48 \\
175 \\
62 \\
92 \\
102 \\
192\end{array}$ & $\begin{array}{r}6 \\
8 \\
47 \\
27 \\
3 \\
20 \\
14 \\
10 \\
8 \\
18\end{array}$ & $\begin{array}{r}43 \\
33 \\
133 \\
118 \\
35 \\
117 \\
45 \\
60 \\
59 \\
97\end{array}$ & $\begin{array}{r}8 \\
6 \\
21 \\
18 \\
5 \\
22 \\
4 \\
8 \\
10 \\
18\end{array}$ & $\begin{array}{r}35 \\
26 \\
192 \\
132 \\
34 \\
142 \\
55 \\
56 \\
60 \\
182\end{array}$ & $\begin{array}{r}4 \\
3 \\
46 \\
27 \\
4 \\
39 \\
11 \\
7 \\
5 \\
19\end{array}$ & $\begin{array}{r}54 \\
44 \\
188 \\
148 \\
35 \\
137 \\
62 \\
80 \\
70 \\
165\end{array}$ \\
\hline A verage & 104 & .... & 99 & & 100 & & 122 & & 74 & & 91 & $\cdots$ & \\
\hline
\end{tabular}

s See table 10 for the composition of the materials.

b Data for only 3 periods of exposure. 
TABLE 15.--Relative maximum penetration of wrought specimens based upon the average maximum penetration of all spscimens for each period

[In percent]

\begin{tabular}{|c|c|c|c|c|c|c|c|c|c|c|c|c|}
\hline \multirow{2}{*}{ Soil No. } & \multicolumn{6}{|c|}{ 2-year exposure a } & \multicolumn{6}{|c|}{ 5-year exposure b } \\
\hline & $A^{\circ}$ & $B$ & $H$ & $N$ & $D$ & $P$ & $A$ & $B$ & $H$ & $N$ & $D$ & $P$ \\
\hline $\begin{array}{l}53 \\
55 \\
58 \\
59 \\
60 \\
61 \\
632\end{array}$ & \begin{tabular}{r}
97 \\
114 \\
63 \\
57 \\
\hdashline 68 \\
48 \\
140 \\
80 \\
154
\end{tabular} & $\begin{array}{r}86 \\
120 \\
68 \\
51 \\
-68 \\
29 \\
200 \\
46 \\
188\end{array}$ & $\begin{array}{r}77 \\
40 \\
205 \\
86 \\
268\end{array}$ & $\begin{array}{r}106 \\
29 \\
177 \\
43 \\
114\end{array}$ & $\begin{array}{r}66 \\
91 \\
57 \\
66 \\
-80 \\
34 \\
120 \\
68 \\
117\end{array}$ & $\begin{array}{r}94 \\
29 \\
148 \\
123 \\
194\end{array}$ & $\begin{array}{r}115 \\
142 \\
118 \\
122 \\
45 \\
68 \\
74 \\
97 \\
39 \\
163\end{array}$ & $\begin{array}{r}127 \\
151 \\
117 \\
115 \\
32 \\
66 \\
66 \\
101 \\
66 \\
156\end{array}$ & $\begin{array}{r}117 \\
135 \\
117 \\
108 \\
9 \\
38 \\
106 \\
154 \\
88 \\
174\end{array}$ & $\begin{array}{r}90 \\
102 \\
127 \\
185 \\
36 \\
43 \\
97 \\
118 \\
65 \\
133\end{array}$ & $\begin{array}{r}47 \\
61 \\
75 \\
127 \\
5 \\
47 \\
54 \\
83 \\
31 \\
86\end{array}$ & $\begin{array}{r}101 \\
117 \\
276 \\
126 \\
57 \\
120 \\
66 \\
149 \\
156 \\
192\end{array}$ \\
\hline Average... & 91 & 95 & 124 & 89 & 78 & 123 & 98 & 100 & 105 & 100 & 62 & 136 \\
\hline
\end{tabular}

\begin{tabular}{|c|c|c|c|c|c|c|c|c|c|c|c|c|}
\hline \multirow{2}{*}{ Soil No. } & \multicolumn{6}{|c|}{ 7-year exposure ${ }^{c}$} & \multicolumn{6}{|c|}{ 9-year exposure d } \\
\hline & $A$ & $B$ & $H$ & $N$ & $D$ & $P$ & $A$ & $B$ & $H$ & $N$ & $D$ & $P$ \\
\hline $\begin{array}{l}53 \\
55 \\
56 \\
58 \\
60 \\
61 \\
62 \\
63- \\
65\end{array}$ & $\begin{array}{r}108 \\
98 \\
127 \\
118 \\
25 \\
42 \\
62 \\
97 \\
90 \\
155\end{array}$ & $\begin{array}{r}107 \\
84 \\
149 \\
155 \\
21 \\
48 \\
70 \\
110 \\
55 \\
149\end{array}$ & $\begin{array}{r}129 \\
96 \\
157 \\
204 \\
14 \\
22 \\
91 \\
110 \\
145 \\
165\end{array}$ & $\begin{array}{r}76 \\
80 \\
176 \\
155 \\
42 \\
24 \\
89 \\
100 \\
98 \\
117\end{array}$ & $\begin{array}{r}62 \\
72 \\
204 \\
155 \\
20 \\
20 \\
72 \\
101 \\
58 \\
96\end{array}$ & $\begin{array}{r}80 \\
124 \\
217 \\
98 \\
28 \\
87 \\
53 \\
176 \\
125 \\
194\end{array}$ & $\begin{array}{r}63 \\
76 \\
121 \\
149 \\
40 \\
69 \\
77 \\
91 \\
126 \\
128\end{array}$ & $\begin{array}{r}92 \\
106 \\
133 \\
146 \\
35 \\
81 \\
108 \\
127 \\
69 \\
138\end{array}$ & $\begin{array}{r}137 \\
117 \\
183 \\
121 \\
28 \\
77 \\
103 \\
108 \\
84 \\
133\end{array}$ & $\begin{array}{r}74 \\
74 \\
194 \\
138 \\
50 \\
34 \\
121 \\
110 \\
68 \\
141\end{array}$ & \begin{tabular}{r|}
47 \\
78 \\
183 \\
65 \\
18 \\
48 \\
52 \\
73 \\
88 \\
93
\end{tabular} & $\begin{array}{r}88 \\
106 \\
171 \\
140 \\
25 \\
138 \\
93 \\
88 \\
171 \\
165\end{array}$ \\
\hline A verage & 92 & 95 & 113 & 96 & 86 & 118 & 94 & 104 & 109 & 100 & 74 & 118 \\
\hline
\end{tabular}

- Average maximum pit depth, 35 mils.

b Average maximum pit depth, 56 mils.

- Average maximum pit depth, 71 mils.

d Average maximum pit depth, 79 mils.

- See table 10 for the composition of the materials.

TABLE 16.-Average of the relative maximum penetrations of wrought specimens for the four periods of exposure

[In percent]

\begin{tabular}{|c|c|c|c|c|c|c|c|c|c|c|c|c|c|}
\hline \multirow[b]{2}{*}{ Soil No. } & \multicolumn{2}{|c|}{$A=$} & \multicolumn{2}{|c|}{$B$} & \multicolumn{2}{|c|}{$H$} & \multicolumn{2}{|c|}{$N$} & \multicolumn{2}{|c|}{$D$} & \multicolumn{2}{|c|}{$P$} & \multirow[b]{2}{*}{ 离 } \\
\hline & 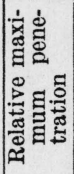 & 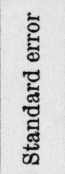 & 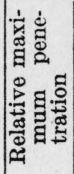 & 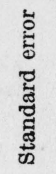 & 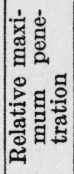 & 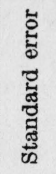 & 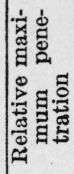 & 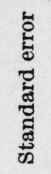 & 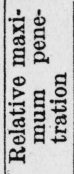 & 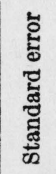 & 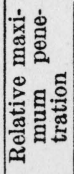 & 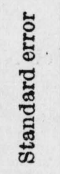 & \\
\hline 58 & $\begin{array}{r}96 \\
108 \\
107 \\
112 \\
37 \\
62 \\
65 \\
106 \\
84 \\
150\end{array}$ & $\begin{array}{r}11 \\
13 \\
15 \\
18 \\
5 \\
6 \\
7 \\
12 \\
17 \\
8\end{array}$ & $\begin{array}{r}103 \\
115 \\
117 \\
117 \\
29 \\
66 \\
68 \\
134 \\
59 \\
158\end{array}$ & $\begin{array}{r}9 \\
15 \\
17 \\
23 \\
3 \\
6 \\
17 \\
23 \\
5 \\
9\end{array}$ & $\begin{array}{r}134 \\
130 \\
128 \\
123 \\
17 \\
54 \\
85 \\
144 \\
101 \\
185\end{array}$ & $\begin{array}{r}9 \\
15 \\
28 \\
30 \\
6 \\
13 \\
24 \\
23 \\
14 \\
29\end{array}$ & $\begin{array}{r}86 \\
93 \\
138 \\
132 \\
43 \\
52 \\
84 \\
126 \\
68 \\
126\end{array}$ & $\begin{array}{r}9 \\
10 \\
31 \\
29 \\
1 \\
18 \\
20 \\
18 \\
12 \\
8\end{array}$ & $\begin{array}{r}56 \\
76 \\
130 \\
103 \\
14 \\
49 \\
53 \\
94 \\
61 \\
98\end{array}$ & $\begin{array}{r}2 \\
5 \\
37 \\
23 \\
5 \\
12 \\
8 \\
11 \\
12 \\
7\end{array}$ & $\begin{array}{r}100 \\
117 \\
207 \\
117 \\
37 \\
110 \\
60 \\
140 \\
144 \\
186\end{array}$ & $\begin{array}{r}11 \\
4 \\
26 \\
9 \\
10 \\
11 \\
14 \\
19 \\
11 \\
8\end{array}$ & $\begin{array}{r}96 \\
106 \\
138 \\
117 \\
30 \\
66 \\
69 \\
124 \\
86 \\
150\end{array}$ \\
\hline Average & 93 & - & 97 & $\ldots$ & 110 & & 95 & & 73 & , n... & 122 & & $\therefore$ \\
\hline
\end{tabular}

- See table 10 for the composition of the materials.

b Data for only 3 periods of exposure. 
Figure 3, which has been prepared from the averages in tables 14 and 16 , indicates that with respect to the relative average values of all materials in the 10 soils for the 4 periods of exposure, nickel-copper steel $(D)$ is better with respect to pitting and loss of weight. However, it should be noted that the mill scale had been removed from these specimens prior to burial. This might have had an efffect on the pitting, as will be shown in a later section when the data of this nickel-copper steel are compared with the data of a steel of a similar
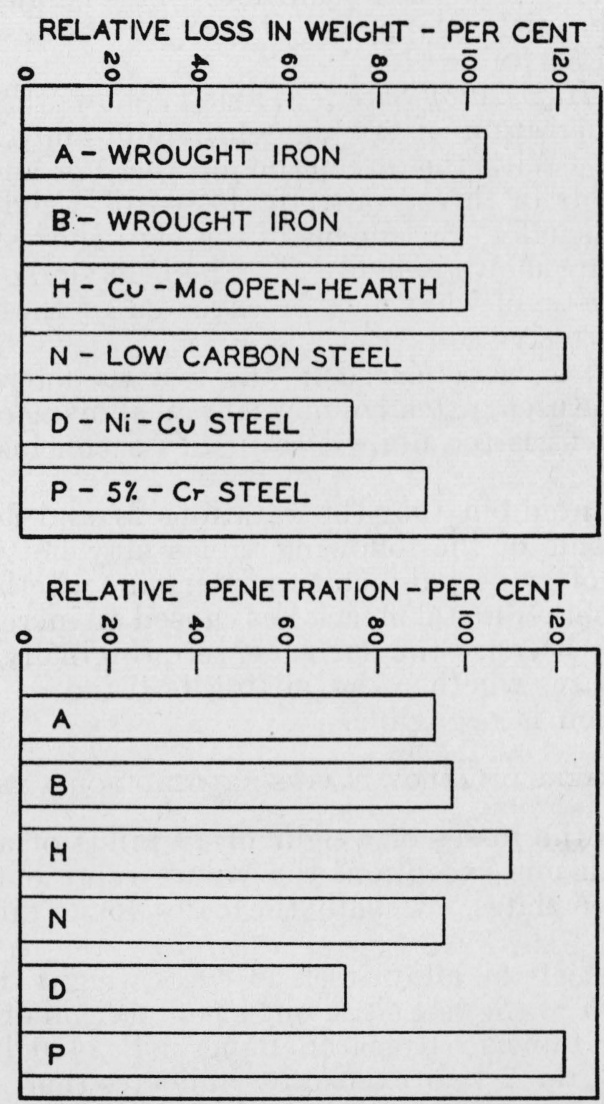

FIGURE 3.-Relative loss of weight and maximum penetration of wrought ferrous materials.

composition from which the mill scale had not been removed. Figure 3 also shows that although the 5-percent chromium steel $(P)$ is superior to ordinary steel with respect to loss of weight, it is inferior with respect to pitting. This may be accounted for by the formation of an adherent chromic-oxide film on the surface of the alloy, which is largely cathodic and behaves temporarily as a protective coating But with the breaking down of this film locally, the resulting differences in potential between the small anodic areas and the cathodic surface tend to cause an acceleration of pitting. Since the rate of corrosion of ferrous metals in soils is usually determined chiefly by 
the rate at which the cathode is depolarized, the relatively large cathodic area would be expected to support a comparatively high rate of pitting. Figure 4 shows the wrought pipe buried in Susquehanna clay (soil 62) for 9 years.

As was pointed out in the introduction, such figures as figure 3, which are based on the averages of the performances of materials that do not behave the same in different soils, have limited value, since the material which shows the best average performance may not be the best for some spectfic soil condition. This is illustrated by the performance of the nickel-copper steel $(D)$ for the last two periods of exposure in soil 56 (table 15).

2. Specimens of High-Alloy Sheet.-Table 17 shows the loss of weight and maximum penetration of six high-chromium-alloy sheets after 9 years of exposure. With the exception of the 12- and 17-percentchromium materials in three soils, the losses of weight and the pit depths were very small. Specimens of the high-alloy sheet buried in soil 64 for 9 years are shown in figure 5, The behavior of these specimens is characteristic of what may be expected of these materials in poorly aerated, corrosive soils.

Materials $S$ and $T$ were placed in the test to determine whether manganese could be used instead of nickel as an alloying element. The number of specimens is too limited to justify a conclusion regarding this.

It should be pointed out that the notations $M$ and $U$ appearing in table 12 and in some of the following tables may not represent real differences. It is often very difficult to determine whether a specimen is unaffected or whether metal attack has caused an increased roughening of the surface, because the surfaces were originally rough before burial. In any case, whether the pitting is listed as $M$ or $U$, the amount of corrosion is negligible.

c) SPECIMENS OF ALLOY PLATES EXPOSED FOR 4 YEARS

Table 18 shows the losses of weight of 10 kinds of alloy iron and steel plates With one exception, the values represent the average loss of weight of two plates. Usually the losses do not differ more than 15 percent.

Apparently most of the alloy steels lost less weight than the openhearth steel in most of the test sites, but a comparison of table 12 with the corresponding table in Research Paper RP 1460 [2], which reports the results of the 2-year exposures, indicates that, although as a whole the performance of the materials was consistent for the two periods, there are a number of cases where the data are inconsistent. Table 19 shows the averages of the deepest pits on these materials. In general, the alloys seem to pit less deeply than plain steel in most of the test sites, but more deeply in several of them. 


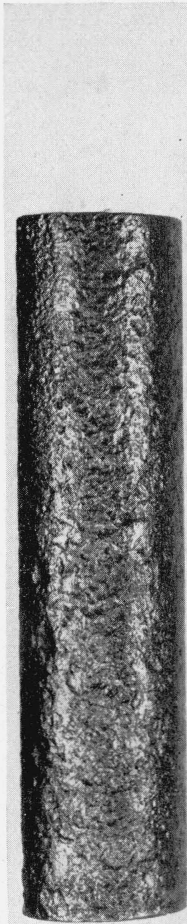

$\mathrm{N}$

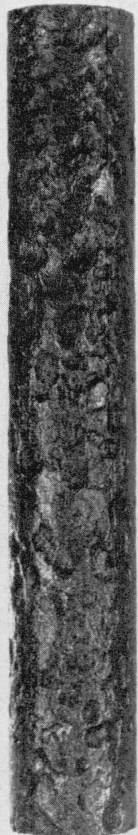

A

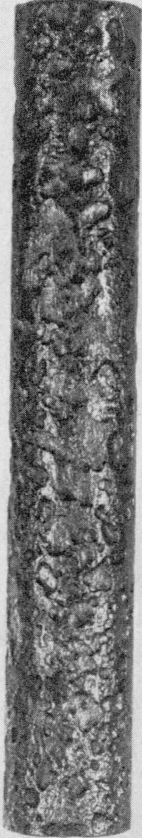

B

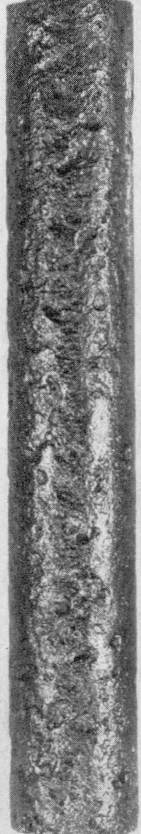

$\mathrm{H}$

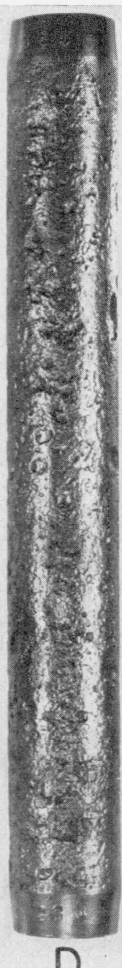

D

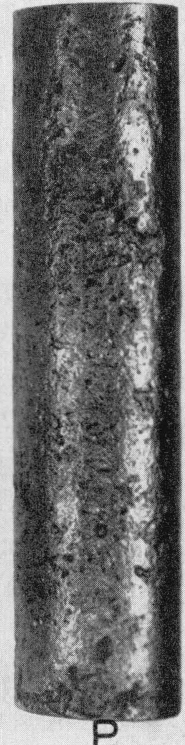

P

FIGURE 4.-Wrought-iron and steel pipe buried 9 years in Susquehanna clay at Meridian, Miss. (soil 62). $N$, Low-carbon tube; $A$, hand-puddled wrought iron; $B$, machine-puddled wrought iron; $H$, copper-molybdenum open-hearth iron; $D$, nickel-copper steel;
\[ , 5 \text {-percent-chromium steel. } \] 

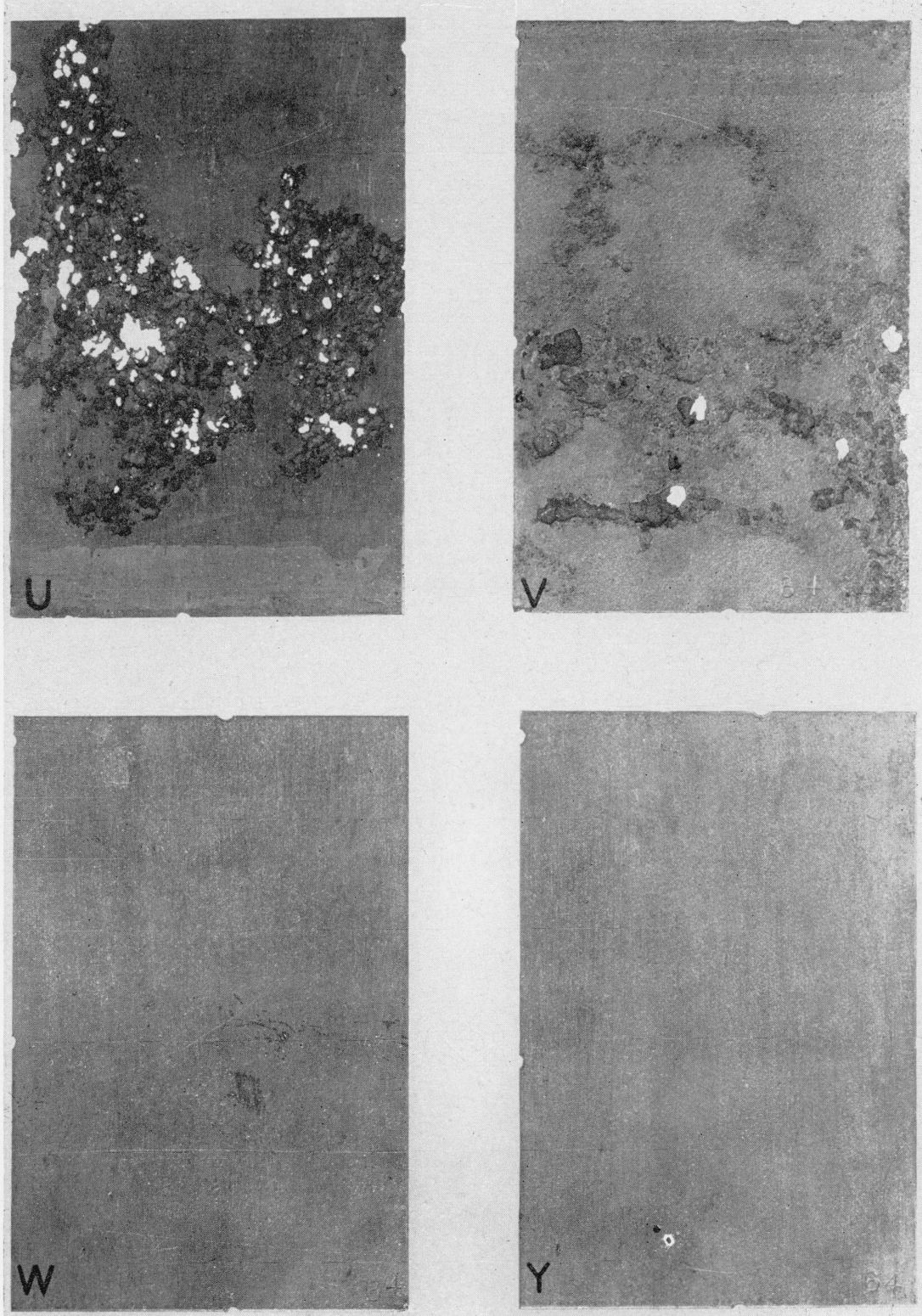

FIGURE 5.- Specimens of high-alloy sheet buried 9 years in Docas clay at Cholame, Calif. (soil 64).

$U$, 12-percent-chromium steel; $V, 18$-percent chromium; $W$, 18-percent-chromium steel with nickel; $Y, 22$ percent-chromium steel with nickel and manganese. The white spots on specimens $W$ and $Y$ are not holes. 
TABLE 17.-Average loss of weight and maximum penetration of high-alloy steel sheets exposed for 9 years

$M$,'shallow metal attack, roughening of surface, but no definite pitting.

$P$, definite pitting, but no pits greater than 6 mils.

$U$, apparently unaffected by corrosion.

contained holes because of corrosion, rendering the computation of the exact penetration impossible. The thickness of the specimen has been used as the maximum pit in this case.

\begin{tabular}{|c|c|c|c|c|c|c|c|c|c|c|c|c|c|c|}
\hline \multirow{3}{*}{ Soil No. } & \multicolumn{2}{|c|}{$\begin{array}{c}(5) \\
11.95 \% \mathrm{Cr} \\
0.48 \% \mathrm{Ni} \\
.38 \% \mathrm{Mn}\end{array}$} & \multicolumn{2}{|c|}{$\begin{array}{c}(5) \\
17.08 \% \mathrm{Cr} \\
0.09 \% \mathrm{Ni} \\
.36 \% \mathrm{Mn}\end{array}$} & \multicolumn{2}{|c|}{$\begin{array}{c}\text { (2) } \\
17.72 \% \mathrm{Cr} \\
9.44 \% \mathrm{Mn}\end{array}$} & \multicolumn{2}{|c|}{$\begin{array}{c}(1) \\
17.76 \% \mathrm{Cr} \\
3.83 \% \mathrm{Ni} \\
6.09 \% \mathrm{Mn}\end{array}$} & \multicolumn{2}{|c|}{$\begin{array}{c}\text { (2) } \\
17.2 \% \mathrm{Cr} \\
8.95 \% \mathrm{Ni} \\
0.44 \% \mathrm{Mn}\end{array}$} & \multicolumn{2}{|c|}{$\begin{array}{c}(5) \\
18.69 \% \mathrm{Cr} \\
9.18 \% \mathrm{Ni} \\
0.36 \% \mathrm{Mn}\end{array}$} & \multicolumn{2}{|c|}{$\begin{array}{c}(5) \\
22.68 \% \mathrm{Cr} \\
12.94 \% \mathrm{Ni} \\
1.50 \% \mathrm{Mn}\end{array}$} \\
\hline & $\begin{array}{l}\text { Loss, av- } \\
\text { erage }\end{array}$ & $\begin{array}{c}\text { Maximum } \\
\text { pene- } \\
\text { tration, } \\
\text { average }\end{array}$ & $\begin{array}{l}\text { Loss, av- } \\
\text { erage }\end{array}$ & $\begin{array}{c}\text { Maximum } \\
\text { pene- } \\
\text { tration, } \\
\text { average }\end{array}$ & $\begin{array}{c}\text { Loss, av- } \\
\text { erage }\end{array}$ & $\begin{array}{l}\text { Maximum } \\
\text { pene- } \\
\text { tration, } \\
\text { average }\end{array}$ & $\begin{array}{l}\text { Loss, av- } \\
\text { erage }\end{array}$ & $\begin{array}{c}\text { Maximum } \\
\text { pene- } \\
\text { tration, } \\
\text { average }\end{array}$ & $\begin{array}{l}\text { Loss, av- } \\
\text { erage }\end{array}$ & $\begin{array}{c}\text { Maximum } \\
\text { pene- } \\
\text { tration, } \\
\text { average }\end{array}$ & $\begin{array}{l}\text { Loss, av- } \\
\text { erage }\end{array}$ & $\begin{array}{c}\text { Maximum } \\
\text { pene- } \\
\text { tration, } \\
\text { average }\end{array}$ & $\begin{array}{c}\text { Loss, av- } \\
\text { erage }\end{array}$ & $\begin{array}{c}\text { Maximum } \\
\text { pene- } \\
\text { tration, } \\
\text { average }\end{array}$ \\
\hline & \multicolumn{2}{|r|}{$U$} & \multicolumn{2}{|r|}{$V$} & \multicolumn{2}{|r|}{$S$} & \multicolumn{2}{|c|}{$T$} & \multicolumn{2}{|c|}{$K$ b } & \multicolumn{2}{|c|}{$W$} & \multicolumn{2}{|c|}{$Y$} \\
\hline \multirow{3}{*}{$\begin{array}{l}53 \\
556 \\
56 \\
58 \\
59\end{array}$} & $o z / f t^{2}$ & Mils & $o z / f t^{2}$ & Mils & $0 z / f t^{2}$ & Mil s & $o z / f t^{2}$ & Mils & $\begin{array}{l}o z / f t^{2} \\
0.0010\end{array}$ & $\underset{U}{\text { Mils }}$ & $o z / f t^{2}$ & Mils & $0 z / f t^{2}$ & Mils \\
\hline & 0.00042 & $M$ & 0.0014 & $\dot{U}$ & & & & & $\begin{array}{l}0.0010 \\
0.110 \\
0.0011\end{array}$ & $\mathrm{~d} \cdot 25+(5,7)$ & 0.00053 & $P$ & 0.0015 & $P$ \\
\hline & .00084 & $P$ & .0016 & $P$ & & & & & .0014 & $M$ & .00074 & $P$ & .0021 & $P$ \\
\hline 60-.... & d.0019 & d $P(5,7)$ & 1.15 & $P(5)$ & & & & & $\begin{array}{l}.0012 \\
.0016\end{array}$ & $\underset{P}{M(5)}$ & .0012 & $P$ & .0024 & $P$ \\
\hline & 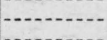 & - & 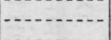 & & - & & & & .0011 & $M$ & & & & \\
\hline & 3.17 & $63+(2,5,7)$ & 1.12 & $63+(2,5,7)$ & 0.48 & $63+(2,5,7)$ & 0.44 & $\mathrm{~d} P(2,7)$ & 8.077 & $114+$ & .0026 & $P$ & .0062 & $P$ \\
\hline $\begin{array}{l}65 \\
66 \\
67\end{array}$ & $\begin{array}{l}0.040 \\
.56\end{array}$ & $\begin{array}{l}53+(2,7) \\
55+(2,5,7)\end{array}$ & $\begin{array}{r}10.24 \\
\quad .70\end{array}$ & d $\begin{array}{c}43+(2,5,7) \\
63+(7)\end{array}$ & .00066 & $\mathrm{~d} M(7)$ & .0042 & $M$ & $\begin{array}{l}.0014 \\
.25 \\
.0015\end{array}$ & 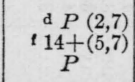 & $\begin{array}{l}.0015 \\
.00053\end{array}$ & $\underset{M}{P(2)}$ & $\begin{array}{l}.0020 \\
.12\end{array}$ & $P(2)$ \\
\hline
\end{tabular}

- The number in parentheses indicates the number of specimens removed from each

test site.

Polished surface.

Data for 1 specimen only.

d A verage loss of weight or pit depth of 1939 removal is greater.

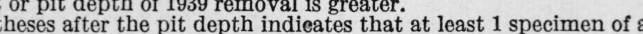

previous removal was punctured, e. g., (2) indicates a puncture after 2 years. 50 percent. 
TABLE 18.- Loss of weight of alloy iron and steel plates exposed for 4 years

[In ounces per square foot]

\begin{tabular}{|c|c|c|c|c|c|c|c|c|c|c|c|c|c|}
\hline \multirow{2}{*}{ 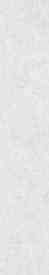 } & \multirow{2}{*}{ Soil } & \multirow{3}{*}{$\begin{array}{l}\text { Expo- } \\
\text { sure }\end{array}$} & \multirow[b]{2}{*}{$\begin{array}{l}\text { Open- } \\
\text { hearth } \\
\text { steel }\end{array}$} & \multicolumn{2}{|c|}{$\begin{array}{c}\text { Open- } \\
\text { hearth iron }\end{array}$} & \multicolumn{4}{|c|}{ Low-alloy steel } & \multicolumn{3}{|c|}{$\begin{array}{c}4 \text { to } 6 \% \text { chromium } \\
\text { steel }\end{array}$} & \multirow[t]{2}{*}{$\begin{array}{l}\text { High } \\
\text { alloy } \\
\text { steel }\end{array}$} \\
\hline & & & & $\begin{array}{l}0.45 \% \\
\mathrm{Cu} \\
.07 \% \\
\mathrm{Mo}\end{array}$ & $\begin{array}{l}0.54 \% \\
\mathrm{Cu} \\
.13 \% \\
\mathrm{Mo}\end{array}$ & $\begin{array}{c}0.95 \% \\
\mathrm{Cu} \\
.52 \% \\
\mathrm{Ni}\end{array}$ & $\begin{array}{c}1.01 \% \\
\mathrm{Cu} \\
1.96 \% \\
\mathrm{Ni}\end{array}$ & $\begin{array}{c}\text { Cr-Si- } \\
\text { Cu-P } \\
\text { steel } \\
1.02 \% \\
\mathrm{Cr}\end{array}$ & $\begin{array}{c}2.01 \% \\
\mathrm{Cr} \\
0.57 \% \\
\mathrm{Mo}\end{array}$ & $\begin{array}{c}5.02 \% \\
\mathrm{Cr}\end{array}$ & $\begin{array}{c}4.67 \% \\
\mathrm{Cr} \\
0.51 \% \\
\mathrm{Mo} \\
.030 \% \\
\mathrm{A1} \\
.022 \% \\
\mathrm{Ti}\end{array}$ & $\begin{array}{c}5.76 \% \\
\mathrm{Cr} \\
0.43 \% \\
\mathrm{Mo} \\
.027 \% \\
\mathrm{A1}\end{array}$ & \\
\hline No. & Type & & $A$ & 0 & $N$ & $J$ & B & $C$ & $K K$ & $D$ & $E$ & $H_{1}$ & $\mathrm{H}_{2}$ \\
\hline $\begin{array}{l}53 \\
55 \\
56 \\
58 \\
60\end{array}$ & $\begin{array}{l}\text { Cecil clay loam } \\
\text { Hagerstown loam.- } \\
\text { Lake Charles clay.- } \\
\text { Muck } \\
\text { Rifle peat. }\end{array}$ & $\begin{array}{l}\text { Years } \\
4.01 \\
3.90 \\
3.99 \\
4.01 \\
3.98\end{array}$ & $\begin{array}{r}3.23 \\
2.58 \\
18.38 \\
9.88 \\
9.52\end{array}$ & $\begin{array}{r}2.90 \\
2.78 \\
13.79 \\
9.87 \\
7.36\end{array}$ & $\begin{array}{r}3.02 \\
2.73 \\
17.37 \\
9.36 \\
8.22\end{array}$ & $\begin{array}{r}1.32 \\
1.81 \\
19.17 \\
10.65 \\
8.62\end{array}$ & $\begin{array}{r}1.07 \\
1.22 \\
19.52 \\
11.01 \\
9.55\end{array}$ & $\begin{array}{r}2.41 \\
2.33 \\
20.76 \\
9.03 \\
10.23\end{array}$ & \begin{tabular}{r|}
2.18 \\
2.38 \\
14.48 \\
8.36 \\
6.00
\end{tabular} & $\begin{array}{r}1.31 \\
1.12 \\
17.93 \\
5.93 \\
6.64\end{array}$ & $\begin{array}{r}1.59 \\
1.19 \\
16.26 \\
7.16 \\
6.23\end{array}$ & $\begin{array}{r}1.44 \\
1.06 \\
14.32 \\
\times 6.81 \\
6.24\end{array}$ & 80.48 \\
\hline $\begin{array}{l}61 \\
62 \\
63 \\
64 \\
65\end{array}$ & $\begin{array}{l}\text { Sharkey clay. } \\
\text { Susquehanna clay. } \\
\text { Tidal marsh } \\
\text { Docas clay } \\
\text { Chino silt loam. }\end{array}$ & $\begin{array}{l}\text { 4. } 01 \\
4.00 \\
4.01 \\
\text { 3. } 98 \\
\text { 3. } 99\end{array}$ & $\begin{array}{r}5.40 \\
3.71 \\
\mathrm{a} 6.23 \\
7.44 \\
5.26\end{array}$ & $\begin{array}{l}5.61 \\
3.62 \\
4.70 \\
8.03 \\
5.00\end{array}$ & $\begin{array}{r}5.65 \\
3.68 \\
4.69 \\
\circ 6.63 \\
5.03\end{array}$ & $\begin{array}{r}4.82 \\
3.31 \\
3.46 \\
\text { c5. } 95 \\
5.00\end{array}$ & $\begin{array}{r}3.94 \\
2.94 \\
3.44 \\
04.86 \\
5.04\end{array}$ & $\begin{array}{l}4.59 \\
\text { 3. } 05 \\
4.14 \\
5.12 \\
5.44\end{array}$ & \begin{tabular}{l|}
4.67 \\
2.99 \\
3.12 \\
5.39 \\
4.53
\end{tabular} & $\begin{array}{r}1.57 \\
1.22 \\
3.80 \\
\text { o } 4.35 \\
\text { c2. } 15\end{array}$ & $\begin{array}{r}1.81 \\
1.09 \\
5.15 \\
5.11 \\
-2.09\end{array}$ & $\begin{array}{r}1.64 \\
1.28 \\
5.41 \\
\text { e5. } 03 \\
\text { e2.17 }\end{array}$ & \\
\hline 66 & $\begin{array}{l}\text { Mohave } f \\
\text { ellyloam. }\end{array}$ & & a16. 82 & & & & 11.38 & & 12.26 & & & & \\
\hline 67 & & & 34.27 & e 33.22 & 27. 70 & 35.06 & 44. 29 & c13. 92 & c16. 45 & & be11. 69 & b12. 88 & \\
\hline $\begin{array}{l}69 \\
70\end{array}$ & $\begin{array}{l}\text { Houghton muck } \\
\text { Merced silt loam }\end{array}$ & $\begin{array}{l}3.98 \\
3.98\end{array}$ & \begin{tabular}{|r|}
4.21 \\
10.63
\end{tabular} \mid & $\begin{array}{r}2.86 \\
\text { b11. } 02\end{array}$ & $\begin{array}{r}2.90 \\
\text { b10.25 }\end{array}$ & $\begin{array}{r}2.61 \\
\text { b } 7.62\end{array}$ & $\begin{array}{l}2.37 \\
7.80\end{array}$ & $\begin{array}{l}2.54 \\
9.80\end{array}$ & $\begin{array}{l}2.46 \\
9.67\end{array}$ & $\begin{array}{r}0.86 \\
\text { b10.04 }\end{array}$ & $\begin{array}{l}0.77 \\
9.88\end{array}$ & \begin{tabular}{|r|}
0.72 \\
10.25
\end{tabular} & \\
\hline
\end{tabular}

a Data for 1 specimen only.

b Data for the individual specimens differ from each other by more than $50 \%$.

- Average loss of weight of the 1939 removals is greater.

Table 20 and figure 6 show the relative losses of weight and pit depths for the materials in all soils based on data for two periods of exposure. The table indicates that in corrosive soils in general the low-alloy materials lose less weight but are not superior to openhearth steel with respect to pit depths. A possible explanation of this condition is that the corrosion products tend to form an imperfect protective coating which sometimes accelerates corrosion at the weak points.

The behavior of the 4- to 6-percent chromium steel in this test is in agreement with the behavior of the 5-percent chromium steel specimens in the 9-year test, where lower loss of weight and deeper pitting was observed in comparison to plain steel.

Further inspection of figure 6 shows that the nickel-copper steel $(B)$ is no better than some of the other low-alloy steels with respect to loss of weight and pitting. This is contrary to the results of the 9-year specimens, where the nickel-copper specimens of approximately the same composition appeared to be somewhat better than the other specimens. It should be noted that the scale on the 9-year nickelcopper steel specimens was removed, whereas, the 4-year specimens were coated with a hard, uniform layer of mill scale. The acceleration of pitting resulting from local failure of the oxide coating would account for the relatively poor performance of the 4-year nickel-copper specimen $(B)$ as compared with the 9-year specimen $(D)$ of the same composition. 
TABLE 19.-Maximum penetration of alloy iron and steel plates exposed for 4 years [In mils]

\begin{tabular}{|c|c|c|c|c|c|c|c|c|c|c|c|c|}
\hline \multirow{3}{*}{ No. } & \multirow{3}{*}{$\begin{array}{l}\text { Soil } \\
\text { Type }\end{array}$} & \multirow{2}{*}{$\begin{array}{l}\text { Open- } \\
\text { hearth } \\
\text { steel }\end{array}$} & \multicolumn{2}{|c|}{ Open-hearth iron } & \multicolumn{4}{|c|}{ Low-alloy steel } & \multicolumn{3}{|c|}{4 to $6 \%$ chromium steel } & \multirow{2}{*}{$\begin{array}{l}\begin{array}{c}\text { High- } \\
\text { alloy } \\
\text { stee1 }\end{array} \\
18 \% \mathrm{Cr}\end{array}$} \\
\hline & & & $\begin{array}{c}0.45 \% \mathrm{Cu} \\
0.07 \% \mathrm{Mo}\end{array}$ & $\begin{array}{l}0.54 \% \mathrm{Cu} \\
0.13 \% \mathrm{Mo}\end{array}$ & $\left|\begin{array}{l}0.95 \% \mathrm{Cu} \\
0.52 \% \mathrm{Ni}\end{array}\right|$ & $\begin{array}{l}1.01 \% \mathrm{Cu} \\
1.96 \% \mathrm{Ni}\end{array}$ & $\begin{array}{c}\mathrm{Cr}-\mathrm{Si}- \\
\mathrm{Cu}-\mathrm{P} \\
\text { steel } \\
1.02 \% \mathrm{Cr}\end{array}$ & $\begin{array}{ll}2.01 \% & \mathrm{Cr} \\
0.57 \% & \mathrm{Mo}\end{array}$ & $5.02 \% \mathrm{Cr}$ & $\mid \begin{array}{l}4.67 \% \mathrm{Cr} \\
0.51 \% \mathrm{Mo} \\
0.030 \% \mathrm{Al} \\
0.022 \% \mathrm{Ti}\end{array}$ & $\begin{array}{c}5.76 \% \mathrm{Cr} \\
0.43 \% \mathrm{Mo} \\
0.027 \% \mathrm{Ti}\end{array}$ & \\
\hline & & $A$ & $O$ & $N$ & $J$ & $B$ & $C$ & $K K$ & & $E$ & $H$ & $\mathrm{H}_{2}$ \\
\hline $\begin{array}{l}53 \\
55 \\
56 \\
58 \\
60 \\
60 .-1\end{array}$ & $\begin{array}{l}\text { Cecil clay loam } \\
\text { Hagerstown loam } \\
\text { Lake Charles clay } \\
\text { Muck. } \\
\text { Rifle peat. }\end{array}$ & $\begin{array}{r}76 \\
54 \\
\text { b } 100 \\
\text { b } 61 \\
40\end{array}$ & $\begin{array}{r}74 \\
44 \\
116 \\
\mathrm{~b} 48 \\
28\end{array}$ & $\begin{array}{r}\text { a } 72 \\
51 \\
100 \\
44 \\
\times 26\end{array}$ & $\begin{array}{r}57 \\
50 \\
96 \\
\text { b } 64 \\
\text { b } 40\end{array}$ & $\begin{array}{r}56 \\
52 \\
\text { b } 139 \\
\text { b } 52 \\
28\end{array}$ & $\begin{array}{r}64 \\
51 \\
\text { b } 77 \\
\text { b } 52 \\
\text { b } 67\end{array}$ & $\begin{array}{r}52 \\
52 \\
\text { b } 60 \\
\text { b } 42 \\
\text { b } 26\end{array}$ & $\begin{array}{r}57 \\
48 \\
95 \\
\text { b i } 44 \\
\text { b } 51\end{array}$ & $\begin{array}{r}50 \\
47 \\
\text { b } 80 \\
\text { b } 46 \\
36\end{array}$ & $\begin{array}{r}57 \\
\text { b } 90 \\
\text { b } 044 \\
32\end{array}$ & 042 \\
\hline $\begin{array}{l}61 \\
62 \\
63 \\
64 \\
65\end{array}$ & $\begin{array}{l}\text { Sharkey clay } \\
\text { Susquehanna clay } \\
\text { Tidal marsh } \\
\text { Docas clay } \\
\text { Chino silt loam }\end{array}$ & $\begin{array}{r}\text { b } 50 \\
47 \\
\circ \quad 26 \\
78 \\
51\end{array}$ & $\begin{array}{r}66 \\
38 \\
\mathrm{Q} 48 \\
75 \\
65\end{array}$ & $\begin{array}{r}54 \\
49 \\
47 \\
76 \\
\times 57\end{array}$ & $\begin{array}{l}63 \\
60 \\
28 \\
84 \\
60\end{array}$ & $\begin{array}{r}\text { i } 56 \\
69 \\
25 \\
88 \\
\times 84\end{array}$ & $\begin{array}{l}41 \\
44 \\
41 \\
70 \\
44\end{array}$ & $\begin{array}{r}35 \\
56 \\
24 \\
70 \\
\mathrm{i} 55\end{array}$ & $\begin{array}{l}36 \\
52 \\
70 \\
60 \\
46\end{array}$ & $\begin{array}{r}36 \\
46 \\
73 \\
\mathrm{~b} 66 \\
48\end{array}$ & $\begin{array}{l}32 \\
58 \\
72 \\
72 \\
56\end{array}$ & 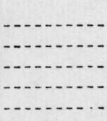 \\
\hline $\begin{array}{l}66 \\
67 \\
69 \\
70\end{array}$ & $\begin{array}{l}\text { Mohave fine gravelly loam.- } \\
\text { Cinders_... } \\
\text { Houghton muck } \\
\text { Merced silt loam. }\end{array}$ & $\begin{array}{c}\text { c d } 188+ \\
\text { a b } \frac{132+}{22} \\
\text { b } 77\end{array}$ & $\begin{array}{r}84 \\
-1 \text { g } 55 \\
20 \\
\times 97\end{array}$ & $\begin{array}{r}\text { i } 98 \\
\text { b } \circ 74 \\
\quad 16 \\
\text { a } 122\end{array}$ & 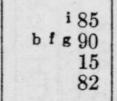 & 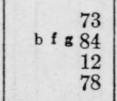 & $\begin{array}{r}\text { b } 80 \\
\text { b } 47 \\
42 \\
\text { b } 94\end{array}$ & $\begin{array}{c}130+ \\
\text { b } 68 \\
27 \\
\text { i } 94\end{array}$ & $\begin{array}{r}\text { b } 99 \\
\text { b } 57 \\
39 \\
121\end{array}$ & $\begin{array}{r}88 \\
\text { b } 52 \\
26 \\
\text { b म } 106\end{array}$ & $\begin{array}{r}117 \\
\text { b } 1 \text { g } 44 \\
33 \\
94\end{array}$ & \\
\hline
\end{tabular}

a Data for the individual specimens differed from each other by more than $50 \%$. b No original surface; impossible to measure true penetration.

d + mark in all cases indic

1 Plate entirely destroyed at 1 end.
g Data for 1 specimen-impossible to obtain data from other specimen because of loss - Severe corrosion at 1 end. f original surface caused by corrosion.

${ }^{1}$ Average pit depths of the 1939 removals are greater. 


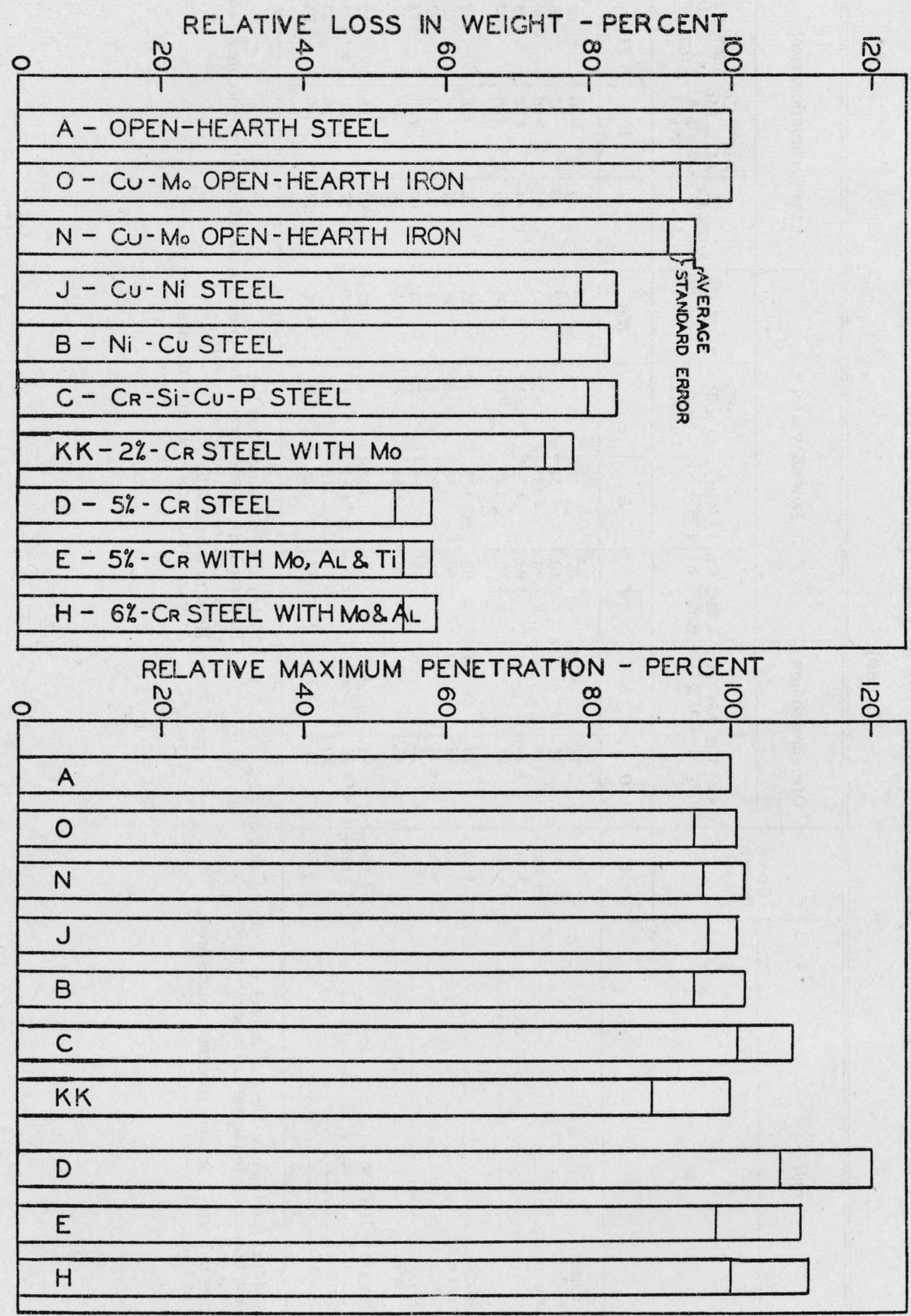

FIGURE 6.-Relative loss of weight and maximum penetration of wrought ferrous materials for two periods of exposure. 


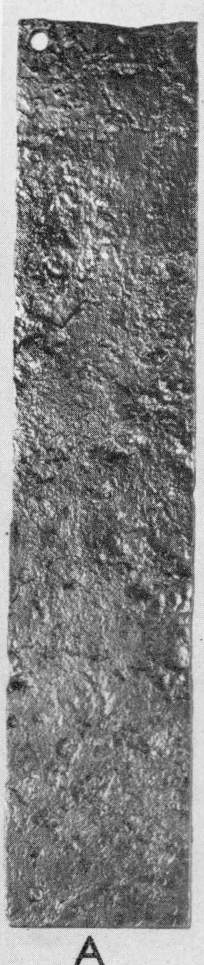

A

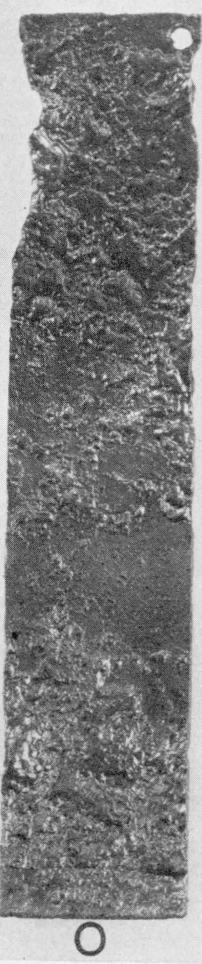

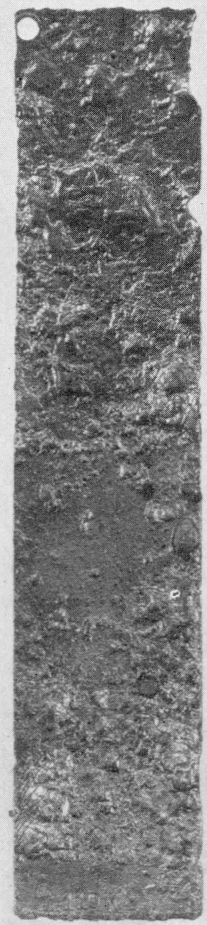

N

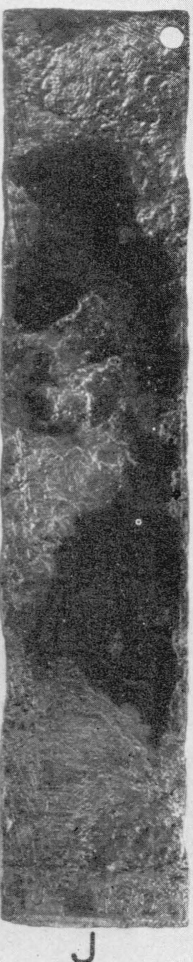

J

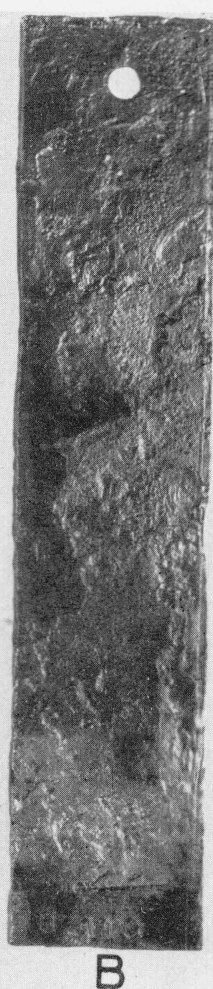

B

Figure 7.-Ferrous plates exposed 4 years to Merced silt loam at Buttonwillow, Calif. See table 10 for the composition of the specimens. 


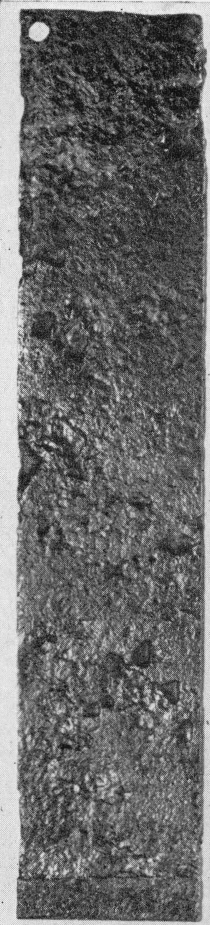

C

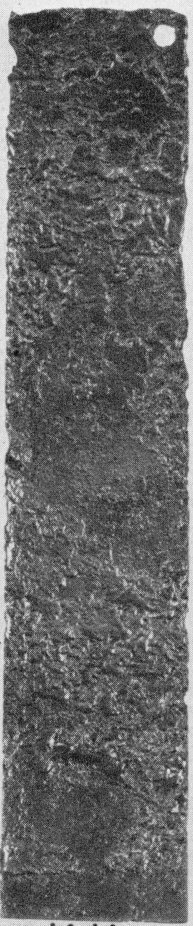

KK

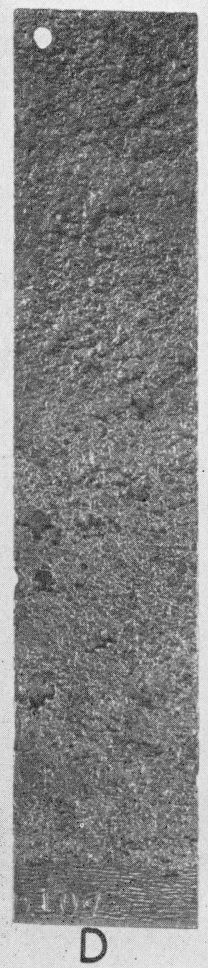

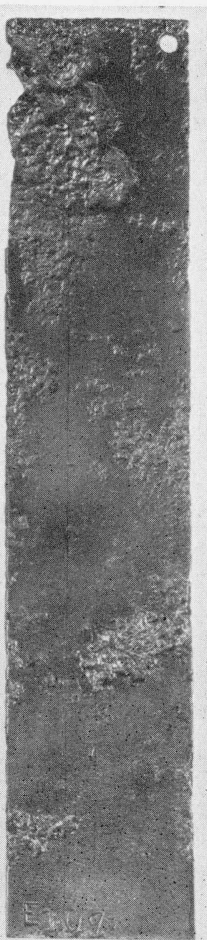

E

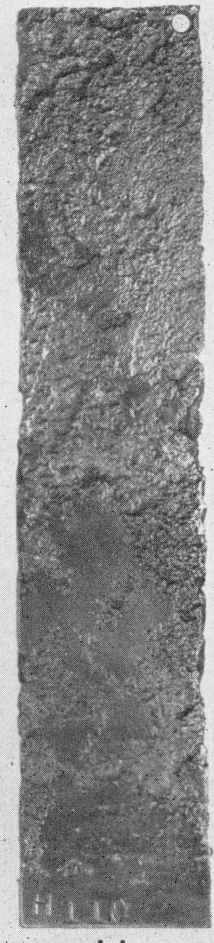

H

Figure 8.-Ferrous plates exposed 4 years to Merced silt loam at Buttonwillow, Calif. See table 10 for the composition of the specimens.

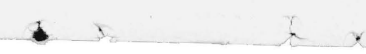


TABLE 20.-Relative loss of weight and maximum penetration of alloy iron and steel plates

[Average of two periods of exposure, in percent]

\begin{tabular}{|c|c|c|c|c|c|c|c|}
\hline \multirow[b]{2}{*}{$\begin{array}{l}\text { Identi- } \\
\text { fica- } \\
\text { tion }\end{array}$} & \multirow[b]{2}{*}{ Material } & \multicolumn{3}{|c|}{ Loss of weight } & \multicolumn{3}{|c|}{ Maximum penetration } \\
\hline & & $\begin{array}{l}\text { Aver- } \\
\text { age }\end{array}$ & $\begin{array}{l}\text { Stand- } \\
\text { ard } \\
\text { devia- } \\
\text { tion }\end{array}$ & $\begin{array}{l}\text { Stand- } \\
\text { ard } \\
\text { error }\end{array}$ & $\begin{array}{l}\text { Aver- } \\
\text { age }\end{array}$ & $\begin{array}{l}\text { Stand- } \\
\text { ard } \\
\text { devia- } \\
\text { tion }\end{array}$ & $\begin{array}{l}\text { Stand- } \\
\text { ard } \\
\text { error }\end{array}$ \\
\hline & Open-hearth steel & 100 & & & 100 & & \\
\hline & Open-hearth iron $(.45 \% \mathrm{Cu} ; .07 \% \mathrm{Mo})$ & 100 & 38 & 7 & 101 & & \\
\hline & Open-hearth iron $(.54 \% \mathrm{Cu} ; .13 \% \mathrm{Mo})$ & 95 & 19 & 4 & 102 & 35 & \\
\hline & Low-alloy $(.95 \% \mathrm{Cu} ; .52 \% \mathrm{Ni})$ & 84 & 25 & 5 & 101 & 24 & \\
\hline & Low-alloy $(1.01 \% \mathrm{Cu} ; 1.96 \% \mathrm{Ni})$ & 83 & 36 & 7 & 102 & 36 & \\
\hline C.... & Low-alloy (Cr-Si-C u-P steel; $1.02 \%$ Cr) & 84 & 19 & 4 & 109 & 45 & \\
\hline & Low-alloy $(2.01 \% \mathrm{Cr} ; .57 \% \mathrm{Mo})$ & 78 & 20 & 4 & 100 & 58 & \\
\hline & 4 to $6 \%$ Cr steel $(5.02 \%$ Cr) & 58 & 27 & 5 & 120 & 70 & \\
\hline & 4 to $6 \%$ Cr steel $(4.67 \%$ Cr; .51\% Mo; $.030 \%$ & 58 & 24 & 4 & 110 & 66 & \\
\hline$H_{-. .}$ & $\begin{array}{l}4 \text { to } 6 \% \text { Cr steel ( } 5.76 \% \text { Cr; } .43 \% \mathrm{Mo} ; .027 \% \\
\text { Al.). }\end{array}$ & 59 & 26 & 5 & 111 & 61 & 11 \\
\hline
\end{tabular}

It is, of course, possible for a material to be much more resistant to corrosion under some soil conditions than to others. For this reason table 20 is not useful for the selection of a material for a specific soil condition. Probably more definite conclusions can be drawn after the remainder of the specimens have been examined.

Figures 7 and 8 show the low-alloy plates exposed to soil 70 . Areas where the mill scale was left intact on the nickel-copper $(B)$ and copper-nickel $(\boldsymbol{J})$ steels are clearly shown.

\section{(d) SPECIMENS EXPOSED FOR 2 YEARS}

Table 21 shows the averages of the losses of weight and maximum penetrations for two low alloys and the two high alloys buried in 1939 . The plates containing 20 percent of chromium and 22 percent of nickel had a peculiar roughening of the surface over large areas when buried, especially near the ends of the specimens, which had the appearance of true pitting. None of these pits measured greater than 6 mils. Therefore, unless definite pits deeper than 6 mils are observed on these plates in future removals, the plates will be classified as unaffected.

Although the losses of weight and depths of maximum penetration of the two high-alloy materials in table 21 are slight, there is a consistent difference favoring the higher alloy. It is too soon to reach definite conclusions as to the relative merits of the copper-molybdenum steel and the chromium-molybdenum steel. The reader is cautioned against comparing these data with data for other materials exposed for the same length of time but buried at an earlier or later date, since two periods equal in length may differ considerably in amount or distribution of rainfall. For longer periods of exposure these differences will be of less importance. 
TABLE 21.-Loss of weight and depth of maximum penetration of low-alloy and high-alloy steel specimens exposed for 2 years

$U$, apparently unaffected by corrosion.

$M$, shallow metal attack, roughening of the surface but no definite pitting.

$P$, definite pitting but no pits on either specimen greater than 6 mils.

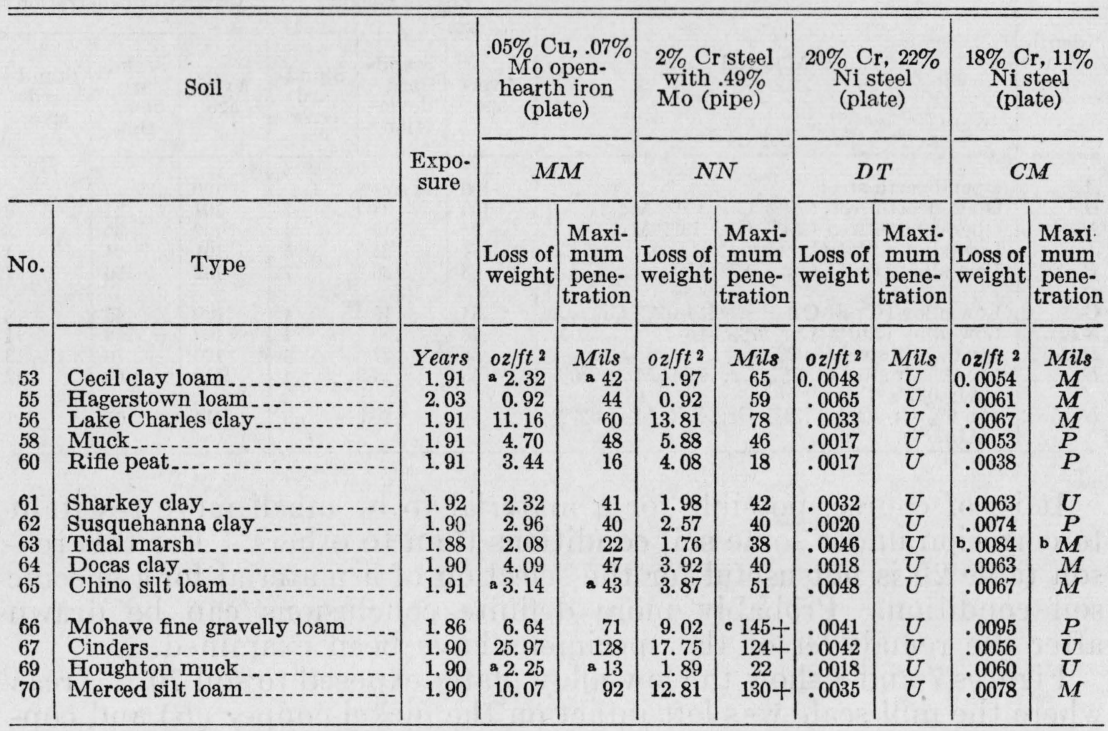

- Data for 1 specimen only.

b Data for 3 specimens.

- + Indicates that 1 or both specimens contained holes due to corrosion.

\section{COPPER AND COPPER-ALLOY}

\section{DIMENSIONS 1 AND $_{2}^{\top}$ COMPOSITION}

Table 22 shows the dimensions and composition of the copper and copper-alloy specimens. It will be noted that the principal alloying elements are zinc, tin, silicon, and nickel. With one exception, the specimens are sections of pipe, the ends of which were closed. 
TABLE 22.-Dimensions and composition of copper and copper-alloy specimens

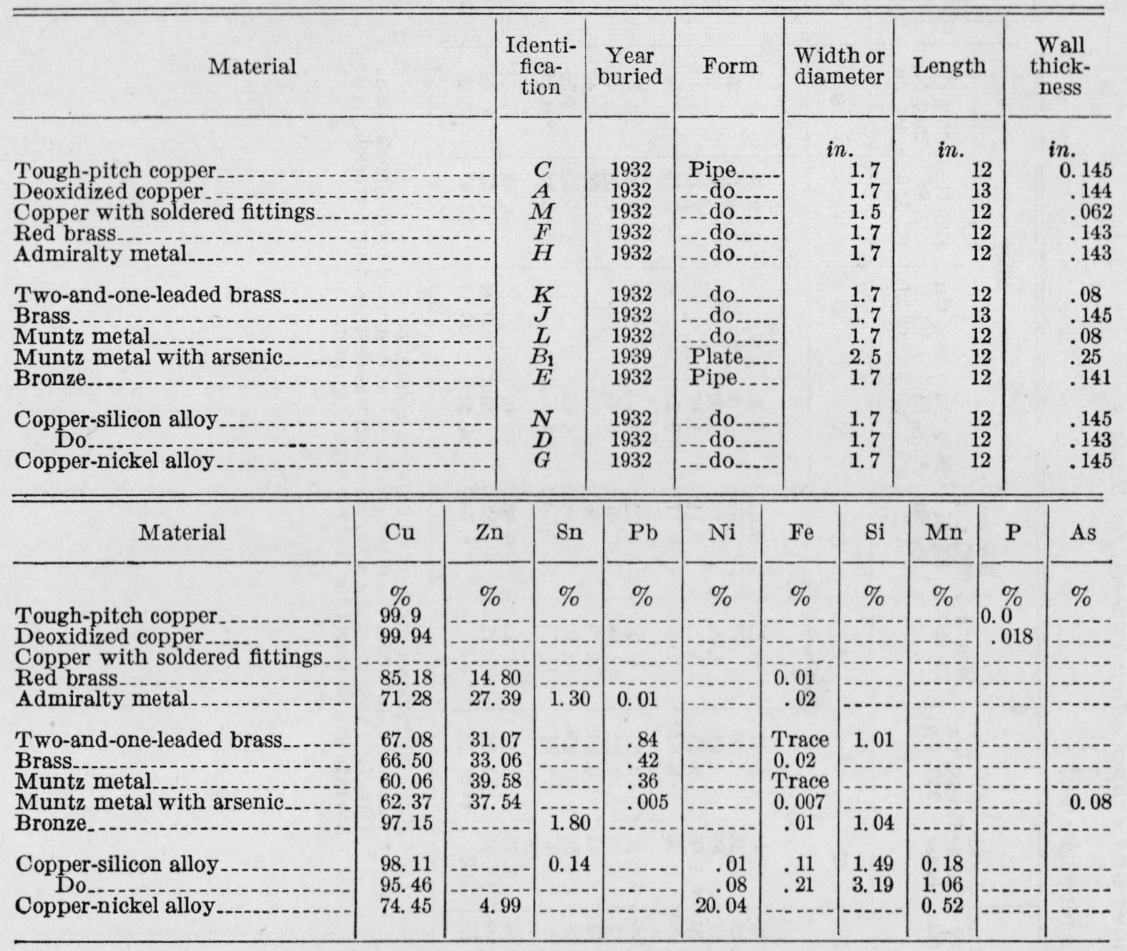

\section{SPECIMENS EXPOSED FOR 9 YEARS}

Table 23 shows the losses of weight of the copper and copper-alloy specimens exposed for 9 years. As a basis for comparison, the losses of open-hearth-steel specimens in the same soils have been added. It will be noted that in most cases the losses of weight of the copper and copper-alloy specimens were less than 10 percent of that of the steel. Table 24 shows the condition of the copper and copper-alloy specimens with respect to pitting. The corrosion of these specimens is much more uniform than that of steel, and the maximum pit depths are much less. Apparently these statements do not apply to the specimens in Rifle peat, soil 60 . This is a very acid organic soil. For some reason open-hearth steel does not pit badly in this soil, although it loses considerable weight. In considering the relative merits of ferrous and nonferrous pipe, it should be remembered that some of the nonferrous pipe is frequently made with thinner wall thickness than is standard for steel pipe of the same diameter, although some materials, such as red brass, are always furnished in standard steel pipe thickness. 
TABLE 23.-Loss of weight of copper and copper alloys exposed for 9 years

[In ounces per square foot]

\begin{tabular}{|c|c|c|c|c|c|c|c|c|c|c|c|c|c|c|c|}
\hline \multicolumn{2}{|r|}{ Soil } & \multirow{3}{*}{$\begin{array}{c}\text { Ex- } \\
\text { posure }\end{array}$} & \multirow{2}{*}{$\begin{array}{l}\text { Tough } \\
\text { pitch } \\
\text { copper }\end{array}$} & \multirow{2}{*}{$\begin{array}{l}\text { Deoxi- } \\
\text { dized } \\
\text { copper }\end{array}$} & \multirow[b]{2}{*}{$\underset{\text { brass }}{\text { Red }}$} & \multirow{2}{*}{$\begin{array}{l}\text { Admi- } \\
\text { ralty } \\
\text { metal }\end{array}$} & \multirow{2}{*}{$\begin{array}{l}\text { 2-and- } \\
\text { 1-leaded } \\
\text { brass }\end{array}$} & \multirow{2}{*}{$\begin{array}{l}\text { Brass } \\
66 \% \mathrm{Cu} \\
33 \% \mathrm{Zn}\end{array}$} & \multirow[b]{2}{*}{$\underset{\text { metal }}{\text { Muntz }}$} & \multirow{2}{*}{$\begin{array}{l}\text { Bronze } \\
97 \% \mathrm{Cu} \\
1 \% \mathrm{Si} \\
1.8 \% \mathrm{Sn}\end{array}$} & \multicolumn{4}{|c|}{ Alloy } & \multirow[b]{2}{*}{$\begin{array}{l}\text { Low- } \\
\text { carbon } \\
\text { steel }\end{array}$} \\
\hline \multirow[t]{2}{*}{ No. } & \multirow[t]{2}{*}{ Type } & & & & & & & & & & $\begin{array}{l}98 \% \mathrm{Cu} \\
1.5 \% \mathrm{Si} \\
0.2 \% \mathrm{Mn}\end{array}$ & $\begin{array}{l}98 \% \mathrm{Cu} \\
1.5 \% \mathrm{Si} \\
0.2 \% \mathrm{Mn}\end{array}$ & $\begin{array}{l}95 \% \mathrm{Cu} \\
3 \% \mathrm{Si} \\
1 \% \mathrm{Mn}\end{array}$ & $\begin{array}{c}75 \% \mathrm{Cu} \\
20 \% \mathrm{Ni} \\
5 \% \mathrm{Zn}\end{array}$ & \\
\hline & & & $C$ & $A$ & $F$ & $H$ & $K$ & $\boldsymbol{J}$ & $L$ & $E$ & $N$ & a $N_{1}$ & $D$ & $G$ & $N$ \\
\hline $\begin{array}{l}53 \\
55 \\
56 \\
58 \\
59\end{array}$ & $\begin{array}{l}\text { Cecil clay loam } \\
\text { Hagerstown loam_- } \\
\text { Lake Charles clay-a } \\
\text { Muck } \\
\text { Carlisle muck }\end{array}$ & $\begin{aligned} \text { Years } \\
9.47 \\
9.11 \\
9.42 \\
9.51 \\
9.12\end{aligned}$ & $\begin{array}{r}0.24 \\
.20 \\
.71 \\
1.95 \\
0.12\end{array}$ & $\begin{array}{r}0.22 \\
.18 \\
.78 \\
2.10 \\
0.11\end{array}$ & $\begin{array}{r}0.25 \\
.25 \\
.70 \\
2.06 \\
0.12\end{array}$ & $\begin{array}{r}0.29 \\
.25 \\
.57 \\
2.09 \\
\cdot 0.086\end{array}$ & $\begin{array}{r}0.47 \\
.36 \\
\text { b } 1.89 \\
0.20 \\
0.20\end{array}$ & $\begin{array}{l}0.41 \\
.30 \\
.94 \\
3.33 \\
0.029\end{array}$ & $\begin{array}{l}0.52 \\
.77 \\
1.21 \\
5.25 \\
0.031\end{array}$ & $\begin{array}{r}0.37 \\
.35 \\
.76 \\
2.11 \\
0.17\end{array}$ & $\begin{array}{r}0.30 \\
.30 \\
.62 \\
1.97 \\
\mathrm{~d} 0.24\end{array}$ & & $\begin{array}{r}0.36 \\
.28 \\
.63 \\
2.12 \\
0.15\end{array}$ & $\begin{array}{l}0.27 \\
.20 \\
.78 \\
1.40 \\
0.088\end{array}$ & $\begin{array}{r}4.09 \\
3.82 \\
28.76 \\
16.24 \\
4.70\end{array}$ \\
\hline $\begin{array}{l}60 \\
61 \\
62 \\
63 \\
64\end{array}$ & 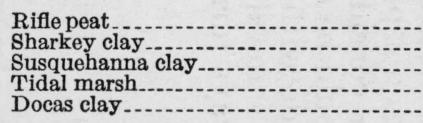 & $\begin{array}{l}9.24 \\
9.53 \\
9.47 \\
9.55 \\
9.21\end{array}$ & $\begin{array}{r}7.26 \\
0.33 \\
.36 \\
4.46 \\
2.80\end{array}$ & $\begin{array}{r}5.01 \\
0.35 \\
\mathbf{4} 48 \\
\mathbf{4} .22 \\
5.32\end{array}$ & $\begin{array}{r}3.55 \\
0.41 \\
\mathrm{i} .43 \\
\mathrm{~d} 1.12\end{array}$ & $\begin{array}{r}\mathrm{d} .85 \\
0.51 \\
.53 \\
.18 \\
.98\end{array}$ & $\begin{array}{r}\text { d } 3.22 \\
0.87 \\
.71 \\
\text { d. } 52 \\
\text { d } 1.07\end{array}$ & $\begin{array}{c}\text { d } 4.21 \\
\text { i } 1.22 \\
0.94 \\
.076 \\
\text { i } 1.94\end{array}$ & $\begin{array}{r}\text { d } 4.56 \\
\text { i } 2.58 \\
1.79 \\
0.10 \\
11.53\end{array}$ & $\begin{array}{r}\mathrm{d} 3.22 \\
0.61 \\
\mathrm{~d} 4.69 \\
4.38 \\
2.30\end{array}$ & $\begin{array}{r}\text { d } 3.96 \\
0.48 \\
.57 \\
6.96 \\
4.87\end{array}$ & 1.82 & $\begin{array}{r}4.46 \\
0.52 \\
.60 \\
5.32 \\
2.88\end{array}$ & $\begin{array}{r}3.61 \\
0.43 \\
.47 \\
3.56 \\
\mathrm{~d} 0.74\end{array}$ & $\begin{array}{r}16.72 \\
5.78 \\
6.65 \\
9.03 \\
\cdot D\end{array}$ \\
\hline $\begin{array}{l}65 \\
66 \\
67\end{array}$ & $\begin{array}{l}\text { Chino silt loam } \\
\text { Mohave fine graveily loam } \\
\text { Cinders... }\end{array}$ & $\begin{array}{l}9.25 \\
9.23 \\
9.24\end{array}$ & $\begin{array}{l}0.26 \\
.45 \\
9.84\end{array}$ & $\begin{array}{r}\text { i } 0.24 \\
\text { di. } 62 \\
11.50\end{array}$ & $\begin{array}{l}0.28 \\
.77 \\
8.46\end{array}$ & $\begin{array}{r}.58 \\
.80 \\
8.27\end{array}$ & $\begin{array}{l}1.39 \\
{ }^{1} 0.95 \\
\left({ }^{1} Z\right)\end{array}$ & $\begin{array}{r}\text { i } 1.60 \\
\text { d } 1.16 \\
\text { gi } 23.10\end{array}$ & $\begin{array}{l}11.45 \\
11.41 \\
\text { ('Z) }\end{array}$ & $\begin{array}{r}\text { i } 0.96 \\
\text { d } 1.53 \\
8.76\end{array}$ & $\begin{array}{l}\text { i } 0.62 \\
\text { d } .51 \\
22.51\end{array}$ & $\begin{array}{r}0.34 \\
23.17\end{array}$ & $\begin{array}{r}\text { i } 0.48 \\
.55 \\
13.47\end{array}$ & $\begin{array}{r}.44 \\
\text { d. } 60 \\
6.28\end{array}$ & $\begin{array}{r}12.86 \\
18.56 \\
\text { в } 58.39\end{array}$ \\
\hline
\end{tabular}

a These specimens had brazed joints; date for 1 specimen only.

b Data for 1 specimen only.

$Z$ indicates specimens destroyed by dezincification.

- Data for 1 specimen; the other specimen was destroyed by dezincification.

d A varage for 3 specimens.
- $D$ indicates specimens destroyed by corrosion.

h Data for 1 specimen; the other specimen was destroyed by corrosion.

A verage loss of weight for 1939 removals is greater. 
$\mathrm{T}_{\mathrm{ABLE}}$ 24.-Maximum penetration of copper and copper alloys exposed for 9 years

[In mils]

\& $M$, shallow metal attack, roughening of the surface but no definite pitting. $P$, definite pitting, no pits greater than 6 mils.

$\mathrm{D}$, selective corrosion, such as dezincification over large areas.
,

$d$, selective corrosion over small areas.

$Z$, specimens destroyed by corrosion (dezincification).

+ , one or both specimens punctured.

\begin{tabular}{|c|c|c|c|c|c|c|c|c|c|c|c|c|c|c|}
\hline \multirow[b]{2}{*}{ Soil No. } & \multirow{2}{*}{$\begin{array}{l}\text { Tough- } \\
\text { pitch } \\
\text { copper }\end{array}$} & \multirow[b]{2}{*}{$\begin{array}{l}\text { Deoxi- } \\
\text { dized } \\
\text { copper }\end{array}$} & \multirow{2}{*}{$\begin{array}{l}\text { Copper } \\
\text { with } \\
\text { soldered } \\
\text { joints }\end{array}$} & \multirow[b]{2}{*}{ Red brass } & \multirow[b]{2}{*}{$\underset{\text { brass }}{\text { Admiralty }}$} & \multirow{2}{*}{$\begin{array}{l}\text { Two- and- } \\
\text { one-leaded } \\
\text { brass }\end{array}$} & \multirow{2}{*}{$\begin{array}{c}\text { Brass } \\
66 \% \mathrm{Cu} \\
33 \% \mathrm{Zn}\end{array}$} & \multirow[b]{2}{*}{$\begin{array}{l}\text { Muntz } \\
\text { metal }\end{array}$} & \multirow{2}{*}{$\begin{array}{l}\text { Bronze } \\
97 \% \mathrm{Cu} \\
1 \% \mathrm{Si} \\
1.8 \% \mathrm{Sn}\end{array}$} & \multicolumn{4}{|c|}{ Alloy } & \multirow{2}{*}{$\begin{array}{l}\text { Low-carbon } \\
\text { steel }\end{array}$} \\
\hline & & & & & & & & & & $\begin{array}{l}98 \% \mathrm{Cu} \\
1.5 \% \mathrm{Si} \\
0.2 \% \mathrm{Mn}\end{array}$ & $\begin{array}{c}98 \% \mathrm{Cu} \\
1.5 \% \mathrm{Si} \\
0.2 \% \mathrm{Mn}\end{array}$ & $\begin{array}{c}95 \% \mathrm{Cu} \\
3 \% \mathrm{Si} \\
1 \% \mathrm{Mn}\end{array}$ & $\begin{array}{c}75 \% \mathrm{Cu} \\
20 \% \mathrm{Ni} \\
5 \% \mathrm{Zn}\end{array}$ & \\
\hline & $C$ & $A$ & a $M$ & $F$ & $H$ & $K$ & $\boldsymbol{J}$ & $L$ & $E$ & $N$ & b $N$ & $D$ & $G$ & $N$ \\
\hline 53 & $\begin{array}{c}6 \\
8 \\
P \\
10 \\
6\end{array}$ & $\begin{array}{r}P \\
P \\
P \\
\cdot 14 \\
P\end{array}$ & $\begin{array}{r}13 \\
8 \\
\mathrm{~d} \\
15 \\
18 \\
9\end{array}$ & $\begin{array}{c}10, D \\
7, D \\
P, D \\
10, D \\
M\end{array}$ & $\begin{array}{r}20, D \\
20, D \\
P, D \\
\mathrm{f} 26, D \\
\text { d } P\end{array}$ & $\begin{array}{l}\mathrm{P}, D \\
7, D \\
P, D \\
\mathrm{~g} P, D \\
P,\end{array}$ & $\begin{array}{r}6, D \\
10, D \\
\mathrm{P}, D \\
8, D \\
M, D\end{array}$ & $\begin{array}{r}6, D \\
6, D \\
P, D \\
6, D \\
P, D\end{array}$ & $\begin{array}{r}12 \\
\circ 20 \\
12 \\
53 \\
20\end{array}$ & $\begin{array}{l}P \\
P \\
P \\
12,8 \\
M\end{array}$ & & $\begin{array}{l}8 \\
8 \\
M \\
12,8 \\
P\end{array}$ & $\begin{array}{l}8, D \\
6, D \\
P, D \\
P, D \\
P\end{array}$ & $\begin{array}{l}59 \\
59 \\
\cdot 154+(7) \\
110, s \\
\cdot 40\end{array}$ \\
\hline 6260 & $\begin{array}{c}40,8 \\
8 \\
8 \\
6 \\
614\end{array}$ & $\begin{array}{c}38,8 \\
8 \\
8 \\
10,8 \\
\cdot 16\end{array}$ & $\begin{array}{c}\cdot 17,8 \\
10 \\
10 \\
6,8 \\
\cdot 13\end{array}$ & $\begin{array}{c}34, D \\
f 7, D \\
14, D \\
6 \\
26, d\end{array}$ & $\begin{array}{l}\text { c } 16, s, d \\
\text { c } 35, D \\
\text { f } 24, D \\
P, d \\
\text { - } 46, D\end{array}$ & $\begin{array}{r}\text { - } 12, D \\
6, D \\
P, D \\
P \\
P \\
\cdot 15, D\end{array}$ & $\begin{array}{r}33, D \\
6, D \\
13, D \\
M, D \\
\mathrm{f} 20, D\end{array}$ & $\begin{array}{l}\text { - } 27, s, D \\
\text { f } 12, D \\
P, D \\
P, D \\
\text { c h } 42+, D\end{array}$ & $\begin{array}{c}18, s \\
\cdot 37 \\
22 \\
10, s \\
34\end{array}$ & $\begin{array}{l}10, s \\
10 \\
P \\
12, s \\
21, s\end{array}$ & $\begin{array}{c}- \\
\cdots \\
\cdots \\
\cdots \\
-\cdots\end{array}$ & $\begin{array}{c}21,8 \\
P \\
6 \\
9 \\
\cdot 16\end{array}$ & $\begin{array}{r}32, D \\
+P, D \\
12, D \\
P \\
\cdot 18, D\end{array}$ & $\begin{array}{l}\circ 27,8 \\
\circ 96 \\
\circ 87 \\
54 \\
154+(5,7)\end{array}$ \\
\hline 65 & $\begin{array}{l}\mathrm{f} \\
\stackrel{P}{P} \\
51,8\end{array}$ & $\begin{array}{l}P \\
10 \\
58,8\end{array}$ & $\begin{array}{l}18 \\
113 \\
145+(5)\end{array}$ & $\begin{array}{r}P, D \\
P, D \\
\cdot 54, D\end{array}$ & $\begin{array}{l}13, D \\
f P, D \\
68,8, D\end{array}$ & $\begin{array}{l}P, D \\
P, D \\
Z(5,7)\end{array}$ & $\begin{aligned} \text { e f } 18, D \\
\quad 8, D \\
\text { i } 132+(2,5,7)\end{aligned}$ & $\begin{array}{l}P, D \\
P, D \\
Z(2,5,7)\end{array}$ & $\begin{array}{r}\mathrm{f} 22 \\
22 \\
102\end{array}$ & $\begin{array}{c}20 \\
13 \\
145+\end{array}$ & $\begin{array}{c}P \\
90, s\end{array}$ & $\begin{array}{c}11 \\
9 \\
80,8\end{array}$ & $\begin{array}{l}P, D \\
P, D \\
36, D\end{array}$ & $\begin{array}{l}112 \\
154+(5,7) \\
154+(2,5,7)\end{array}$ \\
\hline
\end{tabular}

a These specimens had streamlined caps and couplings soldered in place.

- Maximum pits for individual specimens differed from each other by more than 50 percent.

d Data for 3 specimens.

- A number in parentheses after the pit depth indicates that 1 or both specimens of a previous removal was punctured, e. g., (5) indicates that the specimen was puncture after 5 years.
A A verage pit depths of 1939 removals are greater.

h Data for 1 specimen only. pits greater than 8 mils, was badly dezincified, as indicated by the flat sound when the pipe was struck with another piece of metal.
i 1 specimen destroyed by dezincification. 
To facilitate the comparison of materials and minimize the effects of abnormalities in the performance of individual specimens, table 25 has been prepared. In this table the losses of each material for the four periods of exposure have been expressed on a relative percentage basis in terms of the loss of tough pitch copper $C$, which was given the value of 100 percent. The data from soils 63 and 67 were omitted in these averages, the former because the loss of weight of the specimens followed a different trend from that shown by the other soils, the latter because of the complete destruction of the brasses.

TABLE 25.-Reiative loss of weight of copper and copper alloys

[Average of four periods of exposure]

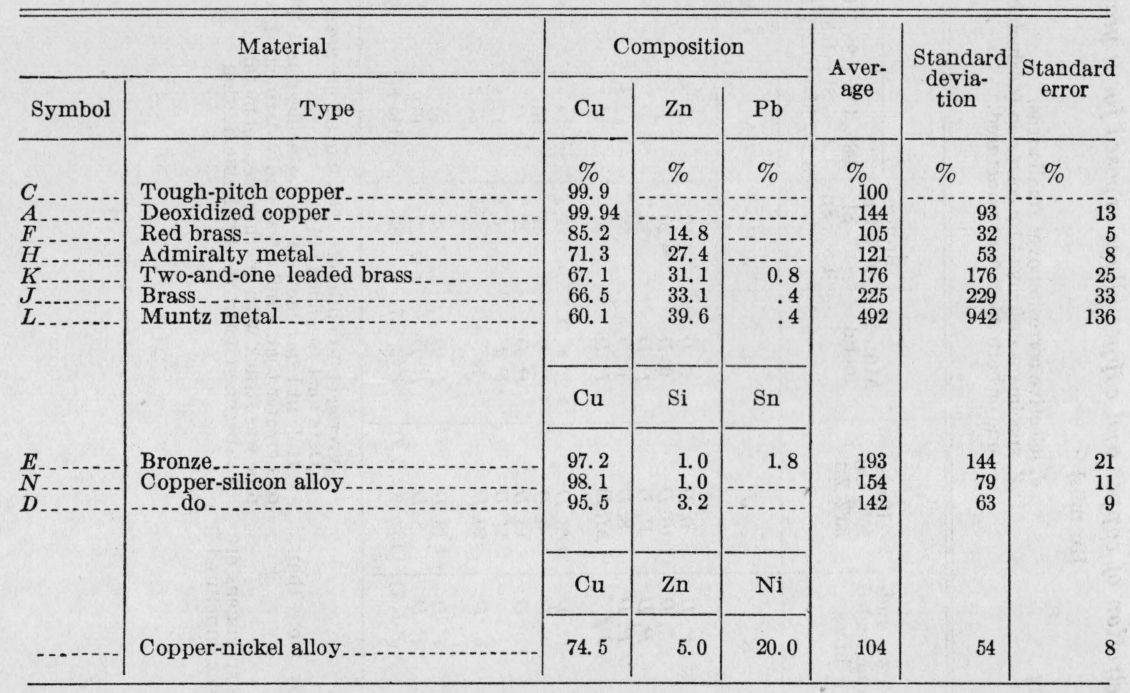

The corrosion losses shown in table 25 in general increase with the increase in zinc content. This is in agreement with statements made previously [2,3], that brasses containing high percentages of zinc are in general much less resistant to corrosion in soils than copper or high-copper alloys. However, a similar table for the specimens exposed to Tidal marsh, soil 63 , would show the reverse. A probable explanation for this difference is the greater resistance of low-copper brasses to sulfides. The relative loss of weight of the deoxidized copper $(A)$ is not in agreement with the other specimens in table 25 , but this may be a result of a mechanical effect, since it is believed that some of these specimens may have been injured in rolling.

In some soils the losses of the brasses do not indicate the extent of corrosion, since part of the zinc was removed by selective corrosion, which weakened the material. Nor do the pit depths for some brasses indicate the extent of corrosion in soils in which dezincification occurred, since the maximum depth of dezincification was not determined except where the specimens were cracked.

It previously has been suggested [2] that because of the degree of consistency shown in a tabulation similar to table 25 for the three periods of exposure, the relative order indicated might be accepted tentatively as the order of merit of the materials for soil conditions 
generally. However, as loss of weight is only one criterion of behavior; consideration would also have to be given to the depth of pitting and the tendency of the material to dezincify. Hence, the order of merit of the brasses shown in table 25 does not show the true comparative worth of the materials because the loss in weight or depth of pitting due to dezincification has not been taken into account. This will be made clearer in the discussion to follow, involving a more detailed examination of some of the brass specimens.

In table 24, selective corrosion, such as dezincification, was reported on most of the brass specimens. The type of corrosion was determined by visual inspection of the specimens before cleaning, which revealed copper-colored spots over large portions of the exposed surface in the attacked areas. Contrary to expectations, selective corrosion was also observed on red brass $(F)$ and the copper-nickel-zinc alloy $(G)$. No differences were evident between the type of corrosion on the brasses in which dezincification is normally expected and that on specimens $F$ and $G$. In order to determine whether any real selective corrosion, such as dezincification, had taken place, a transverse section was cut from a region which showed the copper-colored spots on one of each of the brass and copper-nickel-zinc alloy specimens which were exposed to Sharkey clay, soil 61 . These sections were subjected to a microscopic examination through the courtesy of $\mathrm{H}$. L. Burghoff, research metallurgist of the Chase Brass \& Copper Co. A brief description of the appearance of each of the materials by Mr. Burghoff and his discussion follow:

"Yellow brass pipe $(J)-66.50 \% \mathrm{Cu}, 0.42 \% \mathrm{~Pb}, 0.02 \% \mathrm{Fe}, 33.06 \%$ $\mathrm{Zn}$.-Definite areas of dezincification are visible on the outer surface. A section through one of the most pronounced of these areas shows that dezincification has progressed to about 40 percent of the wall thickness of the pipe. See figure 9 .

"Two-and-one leaded brass tube $(K)-67.08 \% \mathrm{Cu}, 0.84 \% \mathrm{~Pb}, 1.01 \%$ $\mathrm{Si}, 31.07 \% \mathrm{Zn}$.-Dezincification is visible on the outer surface of this specimen. A section through the dezincified region shows that the tube wall has been completely penetrated by the dezincification attack. The microstructure is similar to that of Muntz metal and dezincification begins in the "beta" phase. Corrosion of this material, which is not a commercial pipe material, is more severe than that of specimen $J$. See figures $10, \mathrm{~A}$ and $\mathrm{B}$.

"Muntz metal tube $(L)-60.06 \% \mathrm{Cu}, 0.36 \% \mathrm{~Pb}, 39.58 \% \mathrm{Zn}$.- This specimen has suffered uniform or layer type dezincification over the entire surface. The tube has been corroded considerably, but the dezincified layer is very thin. See figures 11, A and B.

"Admiralty metal $(H)-71.28 \% \mathrm{Cu}, 1.30 \% \mathrm{Sn}, 0.01 \% \mathrm{~Pb}, 0.02 \% \mathrm{Fe}$, $27.39 \% \mathrm{Zn}$. - Some dezincification of this specimen is evident, but its extent is so slight that it can be called superficial. See figure 12 .

"Red Brass $(F)-85.18 \% \mathrm{Cu}, 0.01 \% \mathrm{Fe}, 14.80 \% \mathrm{Zn}$.- Some fine red spots are visible on the roughened surface of this specimen. Microscopic examination shows these to be dezincification, but the extent of this is so slight as to be merely superficial. See figures 13, $\mathrm{A}$ and $\mathrm{B}$.

"Copper-nickel-zinc alloy $(G)-74.45 \% \mathrm{Cu}, 20.04 \% \mathrm{Ni}, 4.99 \% \mathrm{Zn}$, $0.52 \% \mathrm{Mn}$.-Some reddish discoloration is apparent upon the surface. Microscopic examination shows this to be the result of a very super- 


\section{Journal of Research of the National. Bureau of Standards}

ficial selective attack that must involve loss of both nickel and zinc in order to give the resulting copper. See figure 14 .

"It is possible to place the materials into three groups according to the nature of the attack. Red brass $\left(F^{\prime}\right)$, copper-nickel-zinc alloy $(G)$, and Admiralty metal $(H)$, showing superficial selective corrosion, would be in the first group. Yellow brass $(J)$ and the so-called twoand-one leaded brass $(K)$, which is actually a leaded silicon brass, showing definite and deeply penetrating local dezincification, would be in the second group. Muntz metal $(L)$, which suffered the uniform or layer type dezincification, would be in the third group.

"The materials $K$ and $J$ differ in composition somewhat, there being 0.84 percent of lead and about 1 percent of silicon in the $K$ and only 0.42 percent of lead and no silicon in the $J$. There is a very marked difference in the structure of the two alloys. In specimen $J$ there are grains of the alpha phase and particles of lead, the structure being entirely characteristic for yellow-brass pipe. In the $K$ specimen, on the other hand, there are grains of the alpha phase, a "beta" phase, and lead. The occurrence of the "beta" is a result of the presence of silicon. This structure is analogous to that of Muntz metal. Both the materials $K$ and $J$ have suffered dezincification in the soil-corrosion tests. On the basis of the weight loss and depth of pitting shown in tables 23 and 24, and disregarding the dezincification, $K$ may be considered to be somewhat superior to $J$ in soil 61 . The fallacy of this judgment is shown by a study of the photomicrographs of corroded areas of the two materials in figures 10 to 14 . Although exact comparison of the depth of dezincification in the pipe is not possible because of differences in wall thickness of the specimens, it is evident that the yellow brass is superior to the two-and-one leaded brass. Dezincification has completely penetrated the wall of the $K$ specimen over a considerable area, and it is not possible to determine how deeply it would have penetrated if the wall had been heavier. On the other hand, dezincification in the $J$ specimen has penetrated the heavy wall of this pipe by an amount which is definitely less than the wall thickness of the $K$ specimen. The difference in the behavior of the two materials should be ascribed entirely to the silicon present in the leaded silicon brass tube $(K)$ and not to the difference in the amounts of the unimportant (corrosion-wise) lead."

It should be noted that the extent of the corrosion of the red brass, Admiralty and Muntz metals, and the copper-nickel-zinc alloy on the specimens exposed to soil 61 is adequately defined by the observed weight losses and depth of pitting in tables 23 and 24 . On the other hand, the dezincification attack on the yellow brass and on the twoand-one leaded brass is of such extent that it can only be properly evaluated by sectioning the test pieces. Weight losses and depth of pitting have almost no significance in these two cases. These conclusions have been based on the examination of one of each of the brass specimens exposed to Sharkey clay. Since it has previously been shown that soil-corrosion data are sometimes inconsistent, the relationship shown by single specimens in the one soil may not hold in the other soils. Hence, the measurement of the depth of dezincification for all the brasses where this type of attack occurred would unquestionably be highly desirable. This work can not be undertaken at the National Bureau of Standards at present. However, the specimens will be preserved and an attempt will be made to make these measure- 

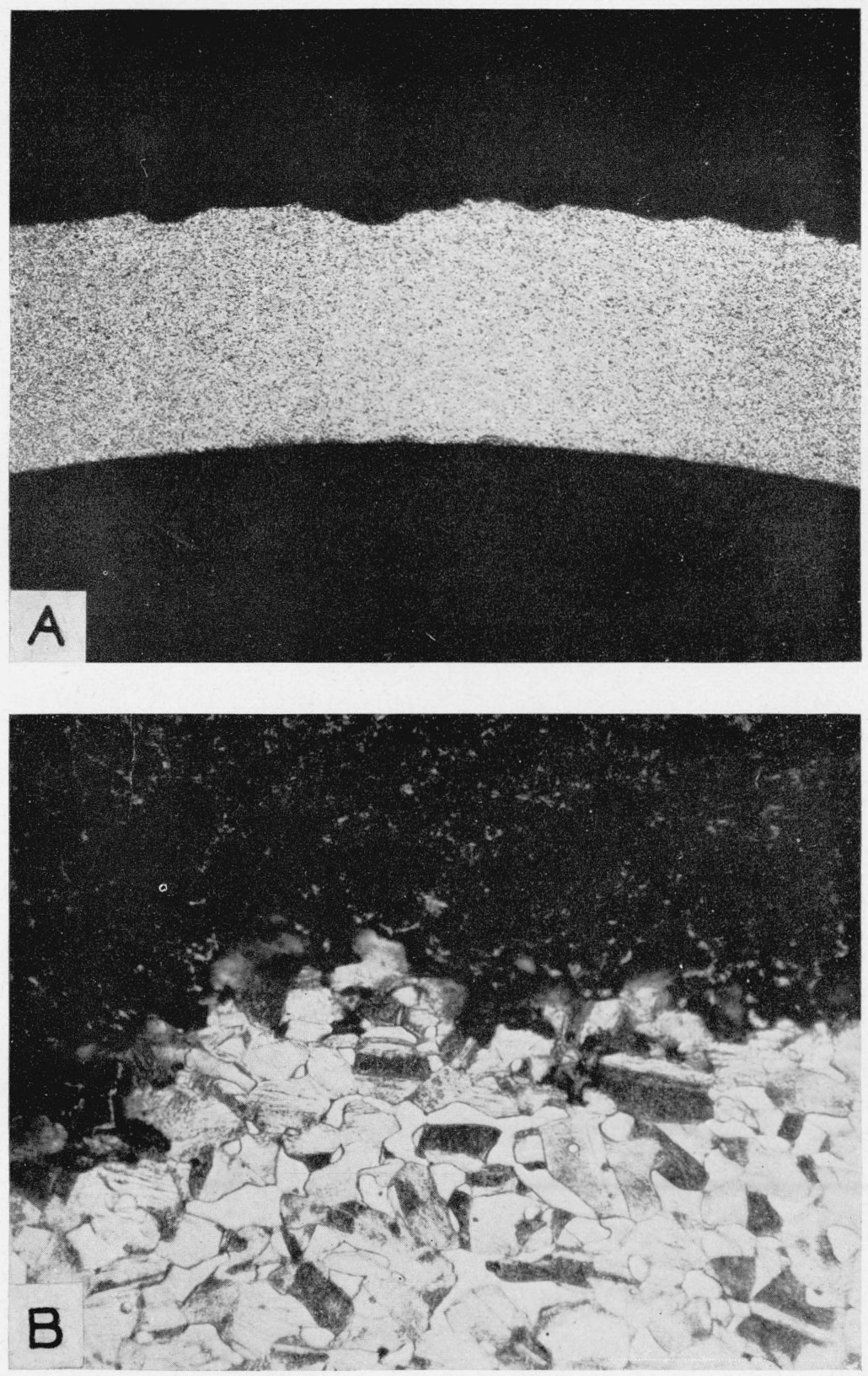

Figure 11.-Muntz metal exposed 9.53 years to Sharkey clay.

$A$, Transverse section showing outer surface roughened by corrosion. The uniformly dezincified layer, which is about 5 percent as thick as the uncorroded metal, is not evident in this photograph. $\times 15, B$, dezincified mass evident in upper portion. $\times 500$. 


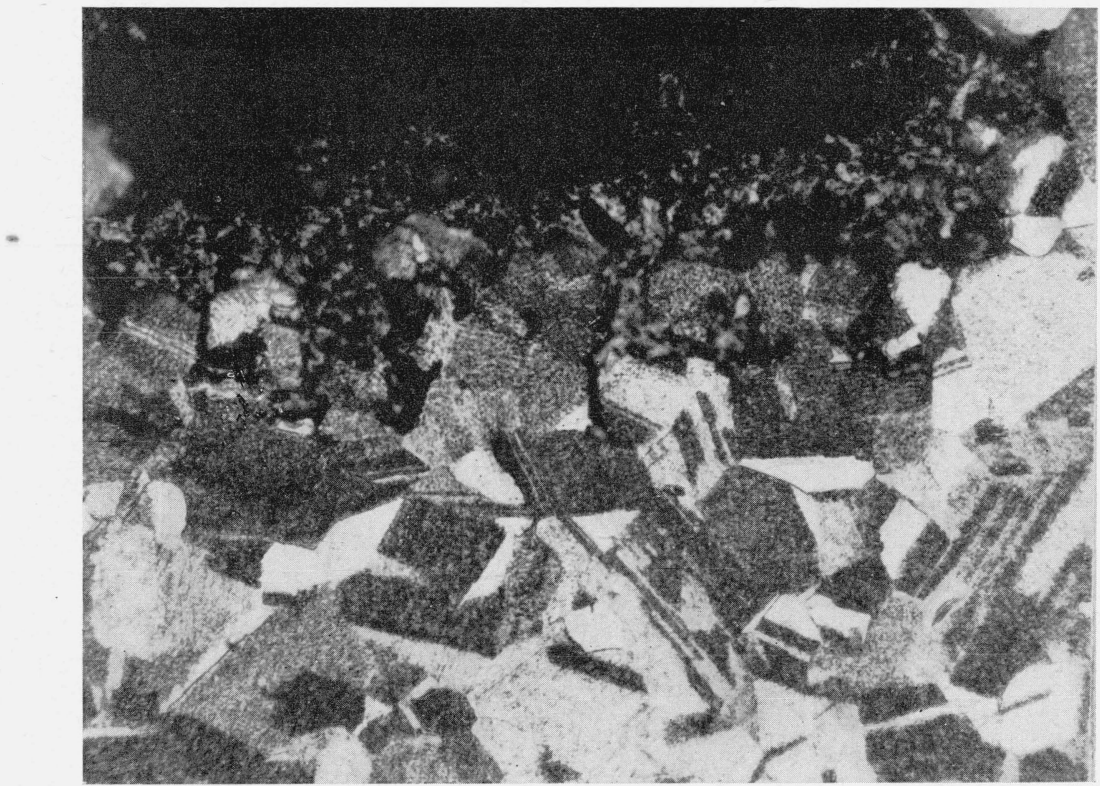

FiguRE 12.-Admiralty metal exposed 9.53 years to Sharkey clay. Transverse section to show thin layer dezincified metal. $\times 500$. 

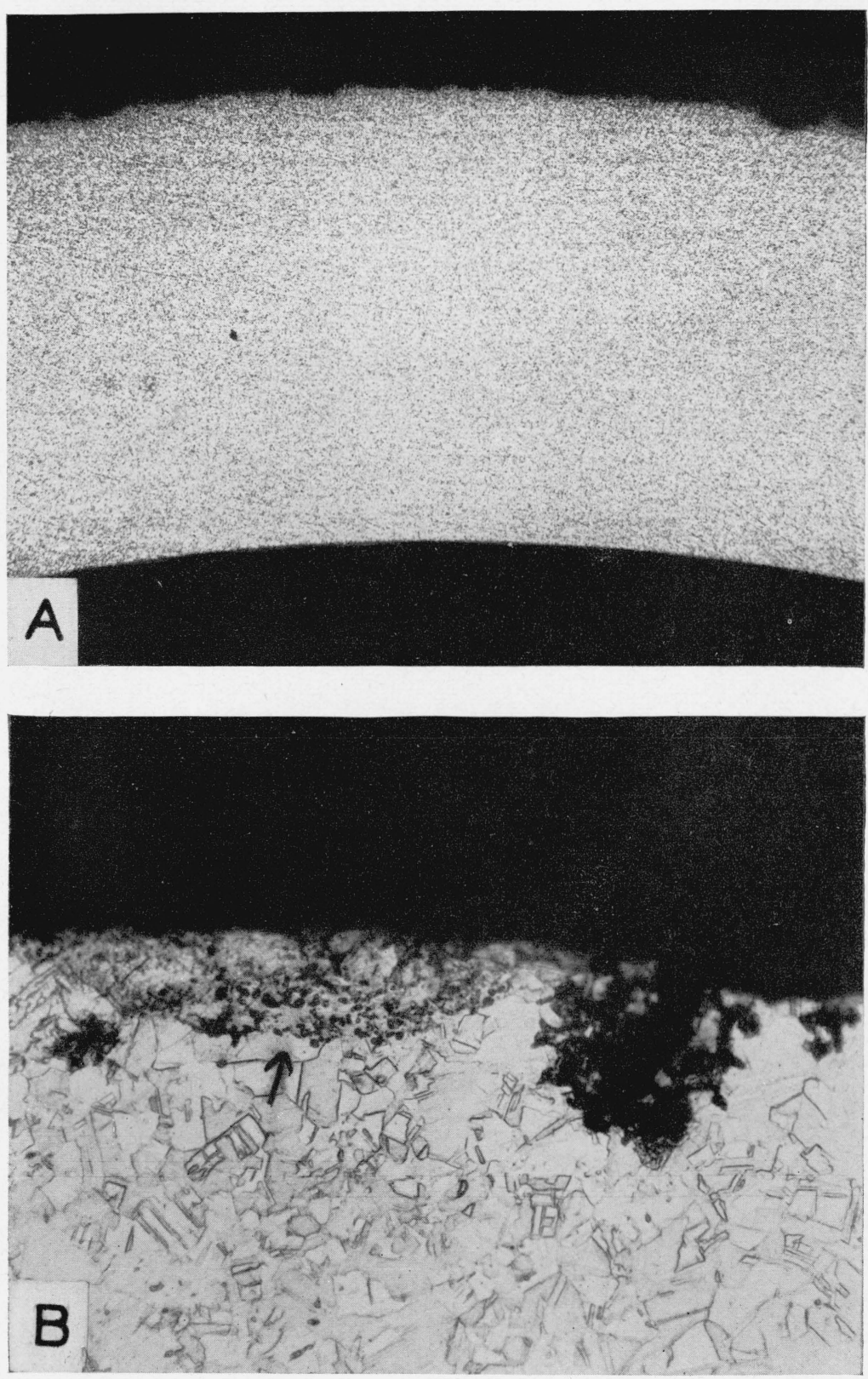

FigURE 13.-Red brass exposed 9.53 years to Sharkey clay.

$A$, Transverse section showing outer surface roughened by corrosion. $\times 15 ; B$, section through pipe wall to the insignificant and superficial character of one dezincified spot included in the area covered by $A$. $\times 500$. 


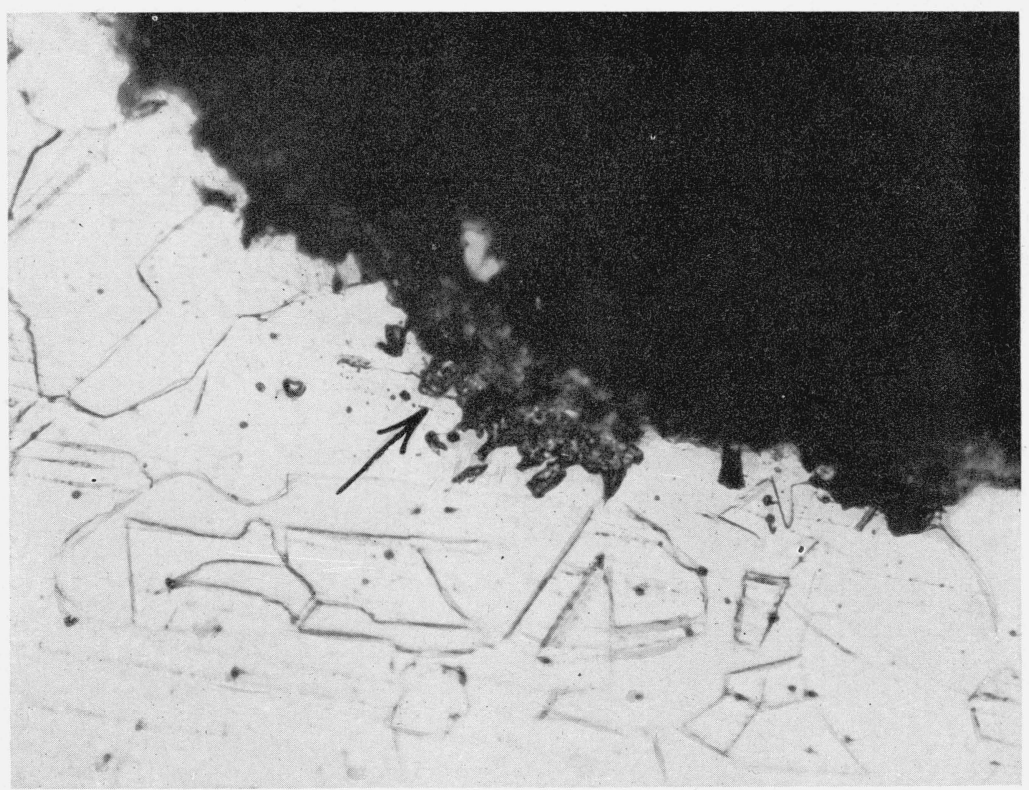

FIGURE 14.-Copper-nickel-zinc-alloy exposed 9.53 years to Sharkey clay. Transverse section showing small mass of copper, a result of very slight selective attack. $\times 500$. 


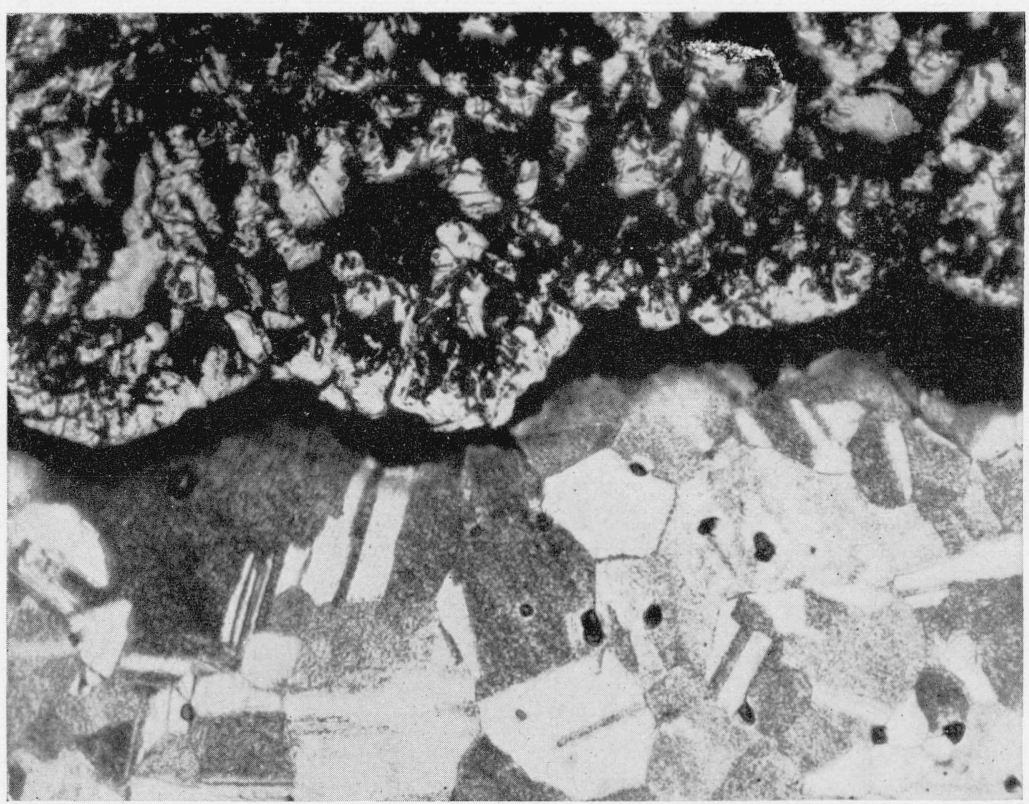

Figure 9.-Yellow brass exposed 9.53 years to Sharkey clay.

Transverse section showing uncorroded metal (below) and dezincified metal (above). $\times 500$. 

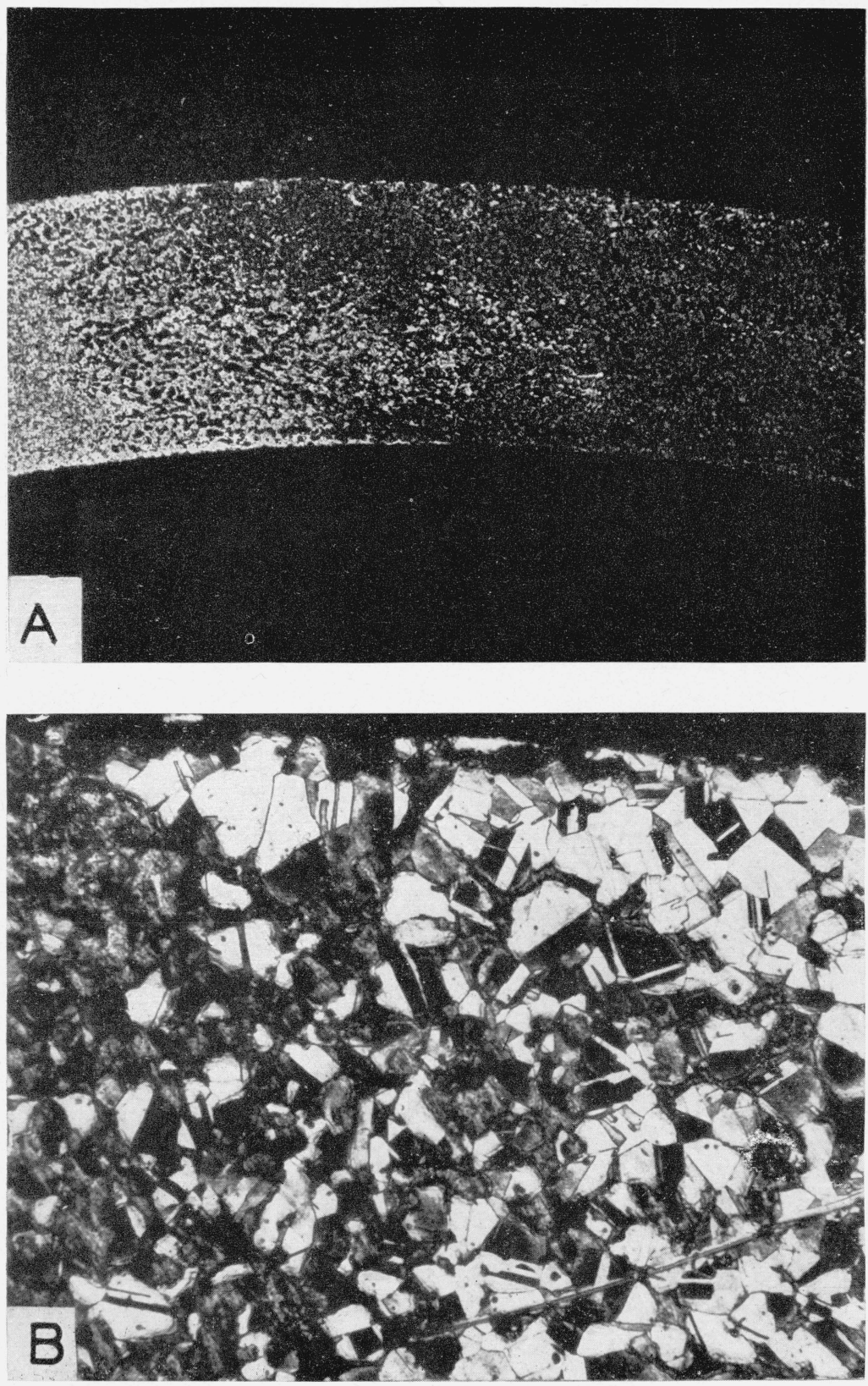

FIGURE 10.-Two-and-one leaded brass (leaded silicon brass) exposed 9.53 years to Sharkey clay.

$A$, Transverse section showing transition from uncorroded metal at left to complete dezincification at right. $\times 15 ; B$, section showing partial dezincification, the dezincification being more severe at the left. $\times 250$. 
ments at a more convenient time in the future. Until such measurements are made, the order of merit of the brasses shown in table 25 should not be taken as indicative of their comparative worth.

\section{MUNTZ METAL SPECIMENS EXPOSED FOR 2 YEARS}

The copper alloy containing approximately 60 percent of copper and 40 percent of zinc, commonly known as Muntz metal, is subject to dezincification under several soil conditions. To determine whether the addition of arsenic to this alloy would prevent dezincification, specimens of Muntz metal plates containing 0.08 percent of arsenic were added to the tests in 1939. Unfortunately, similar plates of ordinary Muntz metal were not buried at the same time. This makes it necessary to compare the behavior of the new materials with that of a section of pipe buried 7 years previously. Obviously, under these conditions small difference in performance may be accidental. Table 26 shows the losses of weight and conditions of the two materials exposed for approximately the same periods. It appears that the addition of 0.08 percent of arsenic was insufficient to prevent dezincification of the brass. Greater resistance to dezincification for this type of material might have been observed if the alloy contained more arsenic. It is known that considerably more arsenic is required to inhibit dezincification in Muntz metal than in alpha brass [20].

TABLE 26.-Loss of weight and maximum penetration of Muntz metal exposed 2 years

$M$, shallow metal attack, roughening of the surface but no definite pitting.

$P$, definite pitting but no pits greater than 6 mils.

$s$, uniform corrosion, no reference surface.

$D$, selective corrosion by dezincification over large areas (several square inchês per square foot).

$\boldsymbol{d}$, selective corrosion over small areas.

$Z$, specimens destroyed by dezincification.

\begin{tabular}{|c|c|c|c|c|c|c|c|}
\hline & Soil & Arser & $\underset{\text { (sheet) }}{\text { nical } \mathrm{Mu}}$ & $\begin{array}{l}\text { ntz metal } \\
B_{1}\end{array}$ & Mun & tz metal & (pipe) $L$ \\
\hline No. & Type & $\begin{array}{l}\text { Expo- } \\
\text { sure }\end{array}$ & $\begin{array}{c}\text { Loss of } \\
\text { weight }\end{array}$ & $\begin{array}{l}\text { Maximum } \\
\text { penetration }\end{array}$ & $\begin{array}{l}\text { Expo- } \\
\text { sure }\end{array}$ & $\begin{array}{l}\text { Loss of } \\
\text { weight }\end{array}$ & $\begin{array}{l}\text { Maximum } \\
\text { penetration }\end{array}$ \\
\hline & & Years & $o z / f t^{2}$ & Mils & Years & $o z / f t^{2}$ & Mils \\
\hline 53 & Cecil clay loam. & 1.91 & 0.18 & $P, D$ & 1.96 & 0.19 & $P, d$ \\
\hline 55 & Hagerstown loam. & 2.03 & .16 & $P, \mathrm{~d}$ & 1.89 & .19 & $6, d$ \\
\hline 56 & Lake Charles clay. & 1.91 & .55 & $12, D$ & 1.99 & .14 & $M, d$ \\
\hline 58 & Muck & 1.91 & .54 & $P, D$ & 1. & .20 & $P, d$ \\
\hline 60 & Rifle peat...... & 1. 91 & 1.87 & $13, D$ & 1. 92 & 1.73 & $9, d$ \\
\hline 61 & Sharkey clay....... & 1.92 & .40 & $P, D$ & .95 & .16 & $P, d$ \\
\hline 62 & Susquehanna clay. & 1.90 & .32 & $P, D$ & 1. 93 & .33 & $P, d$ \\
\hline 63 & Tidal marsh...... & 1.88 & a. 036 & A $P$ & 2.04 & 1.41 & $\mathrm{M}^{\circ}$ \\
\hline 64 & Docas clay & 1.90 & .47 & $P$ & 1.91 & 4.02 & $P, D$ \\
\hline 65 & Chino silt loam & 1.91 & .21 & $P, D$ & 1.91 & 2.43 & $M, D$ \\
\hline 66 & Mohave fine gravelly loam & 1.86 & .30 & $8, d$ & 1. 92 & b. 63 & $\mathrm{~b} P, d$ \\
\hline 67 & Cinders . & 1.90 & 15. 25 & $122, s D$ & 2.02 & $\dot{Z}^{\circ}$ & Z \\
\hline 69 & Houghton muck. & 1.90 & .30 & $P, D$ & & & \\
\hline 70 & Merced silt loam. & 1.90 & .28 & $12, D$ & & & \\
\hline
\end{tabular}

- Data for 3 specimens.

DData for 1 specimen. 


\section{ZINC}

Table 27 shows the dimensions and composition of two kinds of zinc specimens buried in 1937, and table 28 shows the losses of weight and maximum penetration of the specimens, which were exposed for approximately 4 years.

TABLE 27.-Dimensions and composition of zinc specimens

\begin{tabular}{|c|c|c|c|c|c|c|c|c|c|c|c|c|c|}
\hline Material & 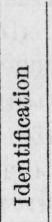 & 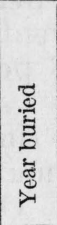 & Form & 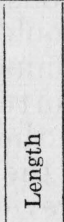 & 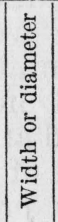 & 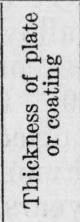 & ষ & $\tilde{J}$ & 实 & $\sum_{\Sigma}^{\infty}$ & $\overrightarrow{\hat{A}_{1}}$ & $\tilde{0}$ & षี \\
\hline $\begin{array}{l}\text { Rolled sinc } \\
\text { Die-casting zinc- } \\
\text { Galvanized steel a }\end{array}$ & $\begin{array}{c}Z \\
C Z \\
T\end{array}$ & $\begin{array}{l}1937 \\
1937 \\
1937\end{array}$ & $\begin{array}{l}\text { Plate.. } \\
\quad \text { do } \\
\text { Pipe... }\end{array}$ & $\begin{array}{l}\text { in. } \\
12 \\
6.81 \\
12\end{array}$ & $\mid \begin{array}{l}\text { in. } \\
2.3 \\
4.44 \\
1.5\end{array}$ & $\begin{array}{l}\text { in. } \\
0.15 \\
.125 \\
.00517\end{array}$ & $\begin{array}{c}\% \\
4.00 \\
.008\end{array}$ & \begin{tabular}{c|c}
$\%$ \\
1.05 \\
-
\end{tabular} & $\begin{array}{c}\% \\
0.009 \\
.018 \\
.07\end{array}$ & $\begin{array}{c}\% \\
0.02 \text { to } 0.05 \\
\end{array}$ & $\begin{aligned} & \% \\
& 0.095 \\
&<.003 \\
& .78\end{aligned}$ & $\begin{array}{c}\% \\
0.0038 \\
<.003 \\
-.\end{array}$ & $\begin{array}{c}\% \\
<0.001 \\
<\end{array}$ \\
\hline
\end{tabular}

Analysis of spelter.

TABLE 28.--Loss of weight and maximum penetration of zinc plates exposed for 4 years

\begin{tabular}{|c|c|c|c|c|c|}
\hline \multicolumn{2}{|r|}{ Soil } & \multicolumn{2}{|c|}{ Rolled zine $\mathrm{Z}$} & \multicolumn{2}{|c|}{ Die-cast zine CZ } \\
\hline No. & Type & $\begin{array}{l}\text { Loss of } \\
\text { weight }\end{array}$ & $\begin{array}{l}\text { Maximum } \\
\text { penetration }\end{array}$ & $\begin{array}{l}\text { Loss of } \\
\text { weight }\end{array}$ & $\begin{array}{l}\text { Maximum } \\
\text { penetration }\end{array}$ \\
\hline $\begin{array}{l}53 \\
55 \\
56 \\
58 \\
60 \\
61 \\
62 \\
63 \\
64 \\
65 \\
66 \\
67 \\
69 \\
70\end{array}$ & $\begin{array}{l}\text { Cecil clay loam } \\
\text { Hagerstown loam } \\
\text { Lake Charles clay } \\
\text { Muck } \\
\text { Rirle peat } \\
\text { Sharkey clay } \\
\text { Susquehanna clay } \\
\text { Tidal marsh } \\
\text { Docás clay } \\
\text { Chino silt loam } \\
\text { Mohave fine gravelly loam } \\
\text { Cinders } \\
\text { Houghton muck } \\
\text { Merced silt loam }\end{array}$ & $\begin{array}{r}\text { oz/ft } 2 \\
0.62 \\
.60 \\
3.42 \\
5.09 \\
10.36 \\
0.96 \\
1.24 \\
\text { b } 2.30 \\
0.57 \\
\text { b } 2.76 \\
\text { d } 12.16 \\
1.70 \\
\text { d } 1.62\end{array}$ & $\begin{array}{c}\text { Mils } \\
10 \\
\text { a } 8 \\
\text { b } 26 \\
66 \\
\text { a } 100 \\
8 \\
9 \\
34 \\
18 \\
36 \\
\text { b } 28 \\
\text { a } 118+(2) \\
10 \\
\text { b } 102+\end{array}$ & $\begin{array}{r}\text { oz/ft }{ }^{2} \\
0.54 \\
.61 \\
4.96 \\
6.33 \\
14.98 \\
1.12 \\
0.60 \\
1.43 \\
2.53 \\
0.76 \\
4.74 \\
13.08 \\
1.64 \\
\text { d } 2.19\end{array}$ & $\begin{array}{c}\text { Mils } \\
22 \\
\text { a } 20 \\
30 \\
\text { c } 125+(2) \\
125+ \\
28 \\
16 \\
24 \\
20 \\
16 \\
124+ \\
125+ \\
36 \\
\text { b } 80+\end{array}$ \\
\hline
\end{tabular}

a Uniform corrosion; no reference surface left.

b Data for individual specimens differed from the average by more than 50 percent.

c+ Indicates that 1 or both specimens punctured by corrision from 1 side of the plate. (2) indicates that 1 specimen from the previous removal was punctured after 2 years.

d Data for 1 specimen only; the other specimen was destroyed by corrosion.

The data are in substantial agreement with similar data obtained after an exposure of 2 years and seem to indicate that with respect to loss of weight and to maximum penetration, the rolled-zinc specimens are superior to the die-cast zinc alloy. The rolled zinc specimens in the tidal marsh corroded more than the others during both periods of exposure. Figure 15 shows specimens of rolled and die-casting zinc exposed to three soils. 


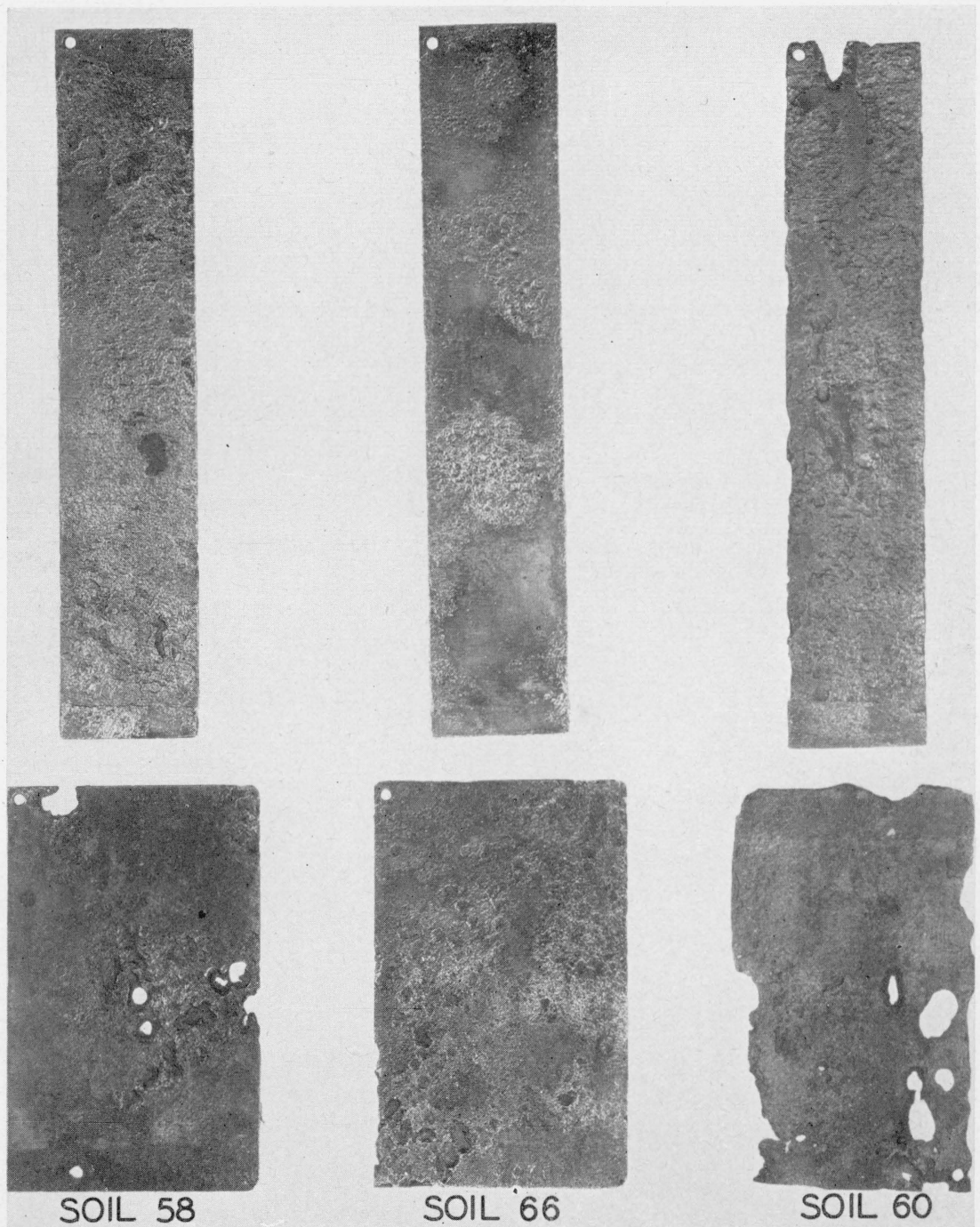

FigURE 15.-Rolled (above) and die-cast (below) zinc buried in muck (soil 58), in Mohave fine gravelly loam (soil 66), and in Rifle peat (soil 60), approximately 4 years each. 


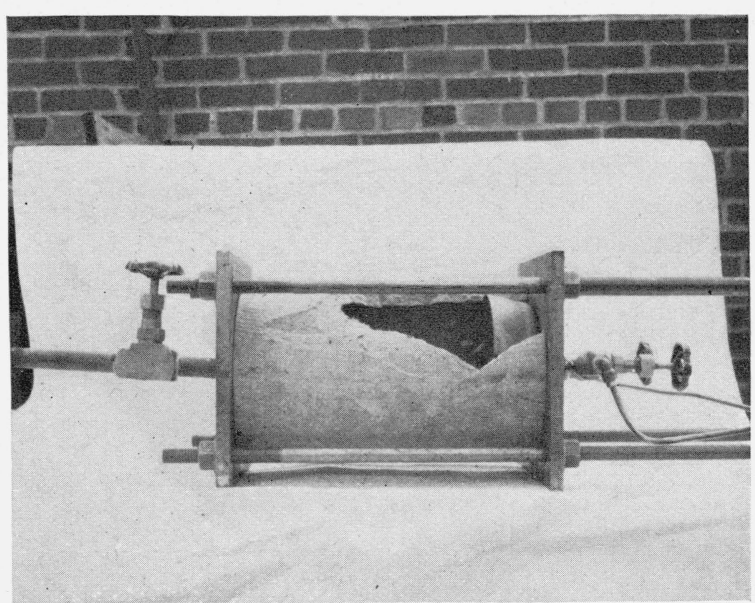

Figure 17.-Bursting test apparatus. 


\section{LEAD}

Three types of lead pipe were buried in 1937 for the purpose of checking the results of an earlier investigation, which tended to show that lead cable sheaths containing antimony corroded somewhat more than sheaths which did not contain this element. The sheaths also differed in source and dimensions.

Table 29 shows the dimensions and composition of lead pipe specimens buried in 1937. The ends of the pipes were closed. Table 30 shows the loss of weight and maximum penetration of the lead specimens after exposures of 2 years and of 4 years. Attention is called to the fact that the pit-depth data for the 2-year specimens has been modified from that previously reported [2]. Because of the softness of the lead pipe, most of the specimens became dented and deformed, and they acquired many tool marks during their removal from the test sites. A recent check of the pit-depth measurements indicated that in many cases pits were recorded that were actually due to the handling of the pipes after they had been removed from the test sites. Therefore, the data in table 30 are to be taken as superseding the data previously published in table 22 of Research Paper RP1460.

TABLE 29.-Dimensions and composition of lead specimens

\begin{tabular}{|c|c|c|c|c|c|c|c|c|c|}
\hline Material & $\begin{array}{l}\text { Identi- } \\
\text { fication }\end{array}$ & $\begin{array}{l}\text { Dia- } \\
\text { meter }\end{array}$ & Length & $\begin{array}{l}\text { Wall or } \\
\text { coating } \\
\text { thickness }\end{array}$ & $\mathrm{Cu}$ & $\mathrm{Bi}$ & $\mathrm{Sb}$ & Sn & $\mathrm{Te}$ \\
\hline $\begin{array}{l}\text { Chemical lead } \\
\text { Tellurium lead } \\
\text { Antimonial lead.... } \\
\text { Lead-coated steel... }\end{array}$ & $\begin{array}{c}\stackrel{O}{T} \\
B \\
C A\end{array}$ & $\begin{array}{r}\text { in. } \\
1.5 \\
1.5 \\
1.5 \\
1.5\end{array}$ & $\begin{array}{l}\text { in. } \\
12 \\
12 \\
12 \\
13\end{array}$ & $\begin{array}{l}\text { in. } \\
0.177 \\
.177 \\
.177 \\
.00144\end{array}$ & $\begin{array}{l}\% \\
0.056 \\
.082 \\
.036\end{array}$ & $\begin{array}{r}\% \\
0.002 \\
\text { None } \\
.016\end{array}$ & $\begin{array}{l}\% \\
0.0011 \\
.0011 \\
5.31\end{array}$ & \begin{tabular}{c}
$a / c$ \\
None \\
do \\
\hdashline do.-... \\
1.00
\end{tabular} & $\begin{array}{c}\% \\
0.043 \\
\end{array}$ \\
\hline
\end{tabular}

The data in table 30 do not show any significant difference for the three varieties of lead pipe. This is not in agreement with the conclusions based on the 2-year exposures [2], where the data indicated a slight improvement by the addition of 5 percent of antimony to the lead. It will be seen in general that soils that are severely corrosive to iron are usually noncorrosive to lead; the chief exceptions are the highly organic soils, such as soils 58 and 61 . The corrosion resistance of lead can be attributed partly to the formation of insoluble compounds on the metal, which protect it from further attack in soils high in salts, such as sulfates, chlorides, and carbonates. The organic salts of lead in organic soils are usually soluble. 
TABLE 30.-Loss of weight and maximum penetration of lead pipe exposed 2 and 4 years

\begin{tabular}{|c|c|c|c|c|c|c|c|c|c|c|c|c|c|c|c|}
\hline \multirow[b]{3}{*}{ No. } & \multirow{3}{*}{ Soil } & \multicolumn{4}{|c|}{ Chemical lead a } & \multicolumn{4}{|c|}{ Tellurium lead b } & \multicolumn{4}{|c|}{ Antimonial lead 0} & \multicolumn{2}{|c|}{$\begin{array}{l}\text { Black iron pipe } \\
\text { exposed } 4 \text { years }\end{array}$} \\
\hline & & \multicolumn{2}{|c|}{ Loss of weight } & \multicolumn{2}{|c|}{$\begin{array}{c}\text { Maximum pene- } \\
\text { tration }\end{array}$} & \multicolumn{2}{|c|}{ Loss of weight } & \multicolumn{2}{|c|}{$\underset{\text { tration }}{\text { Maximum pene- }}$} & \multicolumn{2}{|c|}{ Loss of weight } & \multicolumn{2}{|c|}{$\begin{array}{c}\text { Maximum pene- } \\
\text { tration }\end{array}$} & \multirow{2}{*}{$\begin{array}{l}\text { Loss of } \\
\text { weight }\end{array}$} & \multirow{2}{*}{$\begin{array}{l}\text { Maxi- } \\
\text { mum } \\
\text { pene- } \\
\text { tration }\end{array}$} \\
\hline & & 2 years & 4 years & 2 years & 4 years & 2 years & 4 years & 2 years & 4 years & 2 years & 4 years & 2 years & 4 years & & \\
\hline $\begin{array}{l}53 \\
55 \\
56 \\
58 \\
60\end{array}$ & $\begin{array}{l}\text { Cecil clay loam } \\
\text { Hagerstown loam } \\
\text { Lake Charles clay } \\
\text { Muck } \\
\text { Rifle peat }\end{array}$ & $\begin{array}{r}\text { oz/ft } 2 \\
0.22 \\
.37 \\
.21 \\
1.56 \\
0.18\end{array}$ & $\begin{array}{r}o z / f t t^{2} \\
0.21 \\
.20 \\
.45 \\
2.41 \\
0.28\end{array}$ & $\begin{array}{r}\text { Mils } \\
18 \\
24 \\
38 \\
34 \\
18\end{array}$ & $\begin{array}{r}\text { Mils } \\
12 \\
26 \\
37 \\
28 \\
15\end{array}$ & $\begin{array}{r}o z / f t^{2} \\
0.25 \\
.34 \\
.38 \\
1.68 \\
0.15\end{array}$ & $\begin{array}{r}o z / f t^{2} \\
0.31 \\
.28 \\
.82 \\
2.80 \\
0.20\end{array}$ & $\begin{array}{r}\text { Mils } \\
12 \\
26 \\
30 \\
55 \\
29\end{array}$ & $\begin{array}{r}\text { Mils } \\
20 \\
26 \\
48 \\
56 \\
10\end{array}$ & $\begin{array}{r}o z / f t^{2} \\
0.25 \\
.19 \\
.31 \\
1.45 \\
0.10\end{array}$ & $\begin{array}{r}\text { oz/ft }{ }^{2} \\
0.22 \\
.15 \\
.50 \\
2.12 \\
0.22\end{array}$ & $\begin{array}{r}\text { Mils } \\
10 \\
26 \\
39 \\
50 \\
6\end{array}$ & $\begin{array}{r}\text { Mils } \\
10 \\
18 \\
52 \\
58 \\
\mathrm{~d} P\end{array}$ & $\begin{array}{r}\text { oz/ft }{ }^{2} \\
2.86 \\
2.60 \\
16.03 \\
8.78 \\
8.06\end{array}$ & $\begin{array}{r}\text { Mils } \\
98 \\
50 \\
104 \\
46 \\
38\end{array}$ \\
\hline $\begin{array}{l}61 \\
62 \\
63 \\
64 \\
65\end{array}$ & $\begin{array}{l}\text { Sharkey clay } \\
\text { Susquehanna clay. } \\
\text { Tidal marsh } \\
\text { Docas clay } \\
\text { Chino silt loam }\end{array}$ & $\begin{array}{l}1.46 \\
0.30 \\
.054 \\
.20 \\
.14\end{array}$ & $\begin{array}{l}2.21 \\
0.93 \\
.015 \\
.19 \\
.13\end{array}$ & $\begin{array}{l}35 \\
32 \\
14 \\
24 \\
40\end{array}$ & $\begin{array}{l}39 \\
29 \\
18 \\
16 \\
24\end{array}$ & $\begin{array}{l}1.21 \\
0.36 \\
.056 \\
.25 \\
.17\end{array}$ & $\begin{array}{l}1.75 \\
0.64 \\
\text { e. } 015 \\
.18 \\
.16\end{array}$ & $\begin{array}{l}33 \\
19 \\
10 \\
21 \\
22\end{array}$ & $\begin{array}{r}30 \\
31 \\
-12 \\
11 \\
16\end{array}$ & $\begin{array}{l}.94 \\
.27 \\
.038 \\
.12 \\
.17\end{array}$ & $\begin{array}{l}1.75 \\
1.03 \\
0.013 \\
.19 \\
.21\end{array}$ & $\begin{array}{r}31 \\
12 \\
P \\
12 \\
6\end{array}$ & $\begin{array}{l}42 \\
30 \\
16 \\
12 \\
15\end{array}$ & $\begin{array}{l}4.99 \\
4.30 \\
9.20 \\
5.96 \\
4.56\end{array}$ & $\begin{array}{l}45 \\
56 \\
38 \\
67 \\
59\end{array}$ \\
\hline $\begin{array}{l}66 \\
67 \\
69 \\
70\end{array}$ & $\begin{array}{l}\text { Mohave fine gravelly loam } \\
\text { Cinders } \\
\text { Houghton muck } \\
\text { Merced silt loam }\end{array}$ & $\begin{array}{l}.10 \\
3.67 \\
0.36 \\
.034\end{array}$ & $\begin{array}{r}.10 \\
12.21 \\
0.81 \\
.12\end{array}$ & $\begin{array}{l}44 \\
79 \\
21 \\
48\end{array}$ & $\begin{array}{r}34 \\
104 \\
15 \\
14\end{array}$ & $\begin{array}{l}.25 \\
3.35 \\
0.23 \\
.094\end{array}$ & $\begin{array}{r}.12 \\
13.22 \\
1.08 \\
0.15\end{array}$ & $\begin{array}{r}23 \\
71 \\
8 \\
16\end{array}$ & $\begin{array}{l}41 \\
94 \\
12 \\
27\end{array}$ & $\begin{array}{c}.063 \\
3.14 \\
.20 \\
.10\end{array}$ & $\begin{array}{l}.12 \\
4.21 \\
1.04 \\
0.14\end{array}$ & $\begin{array}{r}12 \\
56 \\
9 \\
11\end{array}$ & $\begin{array}{r}15 \\
90 \\
7 \\
12\end{array}$ & $\begin{array}{r}12.31 \\
\text { в } 37.03 \\
3.28 \\
9.72\end{array}$ & $\begin{array}{c}145+ \\
145+ \\
20 \\
118+\end{array}$ \\
\hline
\end{tabular}

a $\mathrm{Cu}, 0.056 \% ; \mathrm{Bi}, 0.002 \% \mathrm{Sb}, 0.0011 \%$.

- Data for 1 specimen only.

indicates a hole in 1 or both specimens due to corrison.

.

- Data for 1 specimen only. The other specimen was destroyed by corrosion. 


\section{ASBESTOS-CEMENT PIPE}

Asbestos-cement pipe is a mixture of asbestos-fiber and cement. The pipe is built up by a continuous process on a revolving steel mandrel. This is followed by a curing process. Since asbestos-cement pipe is nonmetallic, it is, of course, not subject to galvanic corrosion, tuberculation, or electrolysis. Pipe of this type is manufactured largely for use in transmission mains and services where the working pressures range from 50 to 200 pounds per square inch.

In order to determine whether asbestos-cement pipe is subject to deterioration under soil conditions, specimens were buried at 15 test sites in 1937. The specimens were 12 inches long, 6 inches in diameter, and had an average wall thickness of 0.72 inch. These specimens were cut from class 150 pipe. Two specimens were removed from each test site at each inspection period, after exposure for 2 and for 4 years. In most soils, especially in the acid soils, there was some softening of the surface of the specimens. However, scratching into the specimens showed this softening to be superficial. The softening probably occurred only on the outer layers of the asbestos-cement sheet that were applied to the pipe without pressure during the manufacturing process in order to remove the pipe from the press section. The material immediately under the softened outer layers appeared to be of the same density as the rest of the specimen. In order to measure any change that may have taken place during exposure to the soil, the specimens were subjected to water absorption, density, crushing, and bursting tests. Five representative samples from the group of specimens were stored at the National Bureau of Standards and were subjected to the same tests.

Before the various tests were made on the buried specimens, the 2and 4-year sets were exposed to the air for 4 years and for 2 years, respectively. What effect, if any, this may have had on the results of the tests is not known at present, but a possible explanation may be had from future removals when the specimens will probably be tested immediately after removal from the test sites. In order to reproduce approximately the conditions of the pipe in the soil, the specimens were immersed in water for a least 48 hours previous to making the crushing and bursting tests.

\section{WATER-ABSORPTION TEST}

Weighed air-dried specimens were immersed in water at room temperature for certain periods of time, after which they were removed and wiped with a damp cloth and reweighed. Water absorption was expressed as the percentage gain in weight. The results are tabulated in table 31. 
TABLE 31.-Absorption of water by asbestos-cement pipe

\begin{tabular}{|c|c|c|c|c|c|c|}
\hline \multirow{2}{*}{ Soil No. } & \multirow{2}{*}{$\begin{array}{l}\text { Specimen } \\
\text { No. }\end{array}$} & \multicolumn{5}{|c|}{ Water absorption-percentage gain in weight } \\
\hline & & 1 day & 2 days & 3 days & 4 days & 14 days \\
\hline \multicolumn{7}{|c|}{ 2-YEAR-OLD SPECIMENS } \\
\hline $\begin{array}{l}51 \\
53 \\
56 \\
56 \\
61 \\
62 \\
63 \\
64 \\
66 \\
67 \\
69\end{array}$ & $\begin{array}{r}41 \\
68 \\
149 \\
39 \\
21 \\
71 \\
12 \\
56 \\
11 \\
111 \\
121 \\
99 \\
131 \\
89 \\
101\end{array}$ & 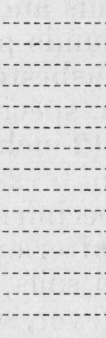 & $\begin{array}{l}1.64 \\
2.13 \\
1.86 \\
.86 \\
3.08 \\
5.21 \\
1.13 \\
1.55 \\
1.50 \\
1.15 \\
1.58 \\
1.11 \\
1.00 \\
1.96 \\
2.05\end{array}$ & $\begin{array}{l}1.87 \\
2.74 \\
2.35 \\
.97 \\
3.86 \\
6.26 \\
1.36 \\
1.81 \\
1.97 \\
1.31 \\
1.69 \\
1.37 \\
1.25 \\
2.48 \\
2.40\end{array}$ & $\begin{array}{l}-1 \\
-1\end{array}$ & $\begin{array}{r}3.68 \\
6.75 \\
6.76 \\
1.56 \\
7.49 \\
10.18 \\
2.79 \\
3.05 \\
4.25 \\
2.70 \\
3.99 \\
4.42 \\
5.10\end{array}$ \\
\hline
\end{tabular}

4-YEAR-OLD SPECIMENS

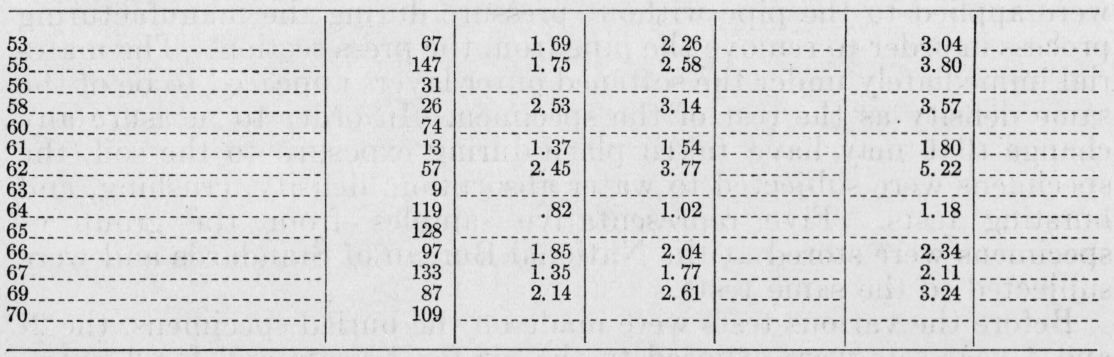

UNBURIED SPECIMENS

\begin{tabular}{|c|c|c|c|c|c|c|}
\hline 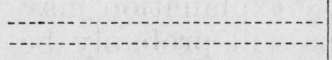 & $\begin{array}{l}2 \\
3\end{array}$ & 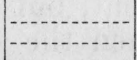 & $\begin{array}{l}\text { 4. } 73 \\
5.96\end{array}$ & $\begin{array}{l}5.69 \\
7.26\end{array}$ & (2) & \\
\hline
\end{tabular}

There is no evident consistency in the amount of water absorbed by the specimens as a group, or by the individual specimens buried in the same soil and removed during different periods. Figure 16 shows some of the representative curves obtained from the data. The curves indicate that the unburied specimens absorbed more water than did the buried specimens, with the possible exception of the 2-year specimen from soil 60 .

\section{CRUSHING TESTS}

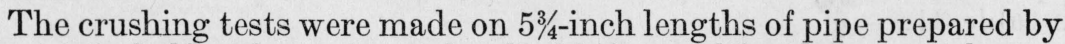
cutting in half each specimen that had been used for the water-absorption tests. After being air-dried these sections were immersed in water for 48 hours. The tests were made with three-edge bearings according to the method described in the Federal specifications for asbestos-cement pipe. Each section was placed in a horizontal position on lower bearings consisting of two wooden strips with vertical sides, each strip having its interior corner rounded to a radius of approximately $1 / 2$ inch. The strips were securely fastened to a rigid wooden block with their interior vertical sides 1 inch apart. The 
upper bearing consisted of a straight wooden block, 4 by 4 inches in cross section. The load was applied at a uniform rate of approximately 1,000 pounds per minute until failure of the pipe occurred. In testing the specimens, the two sections from the same specimen were placed under the hydraulic jack in such a way that the parts of the sections receiving the maximum stress were at an angle of $90^{\circ}$ to each other.

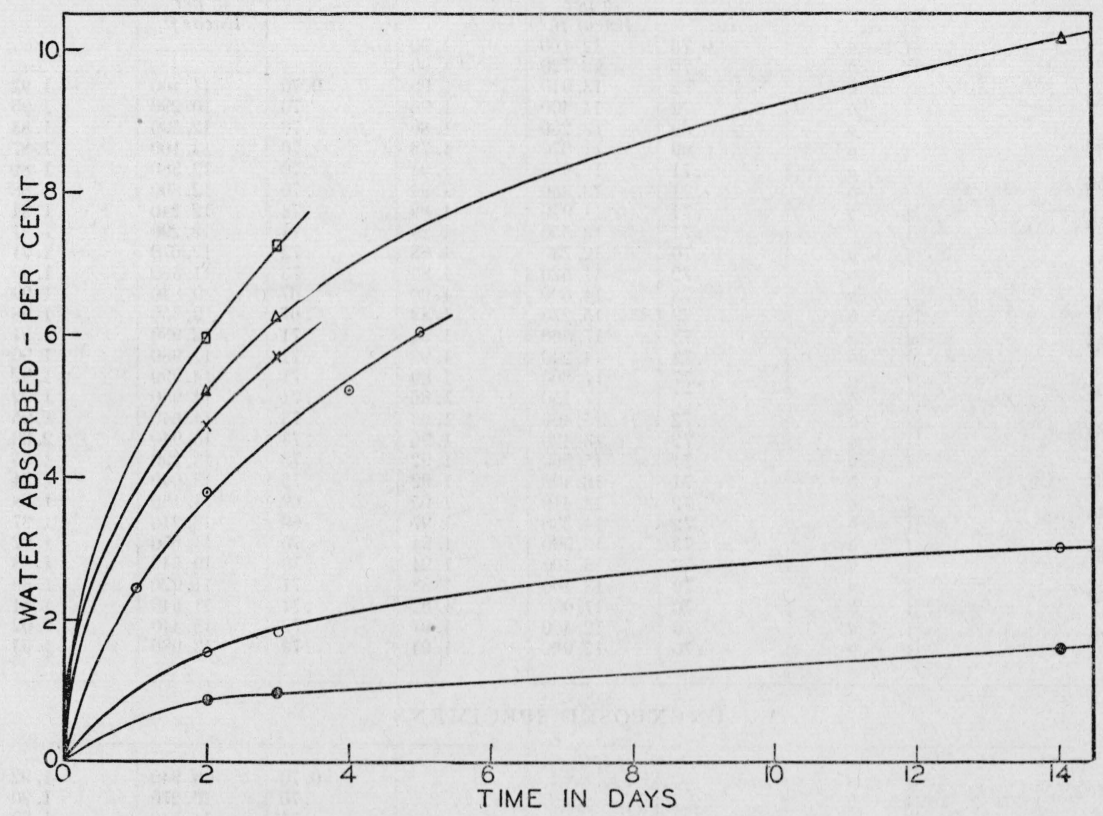

Figure 16.-Percentage of water absorbed by asbestos-cement specimens.

$=$ Exposed 2 years to soil 56 .
$=$ Exposed 2 years to soil 62 . $\odot=$ Exposed 2 years to soil 62 .

$\triangle$
$\widehat{X}=$ Exposed 2 years to soil 60 .

$X=$ Unexposed.
$\square=$ Unexposed.

The crushing strengths in pounds per linear foot for each of the sections were calculated. The results of these tests are given in table 32 for the specimens exposed to the soil and for the unexposed specimens. No evident correlation is shown between the value for the crushing strength and the age of the specimens either in individual soils or as any group of soils. 
TABLE 32.-Results of crushing tests on the asbestos-cement specimens exposed to various soils and on the unexposed specimens

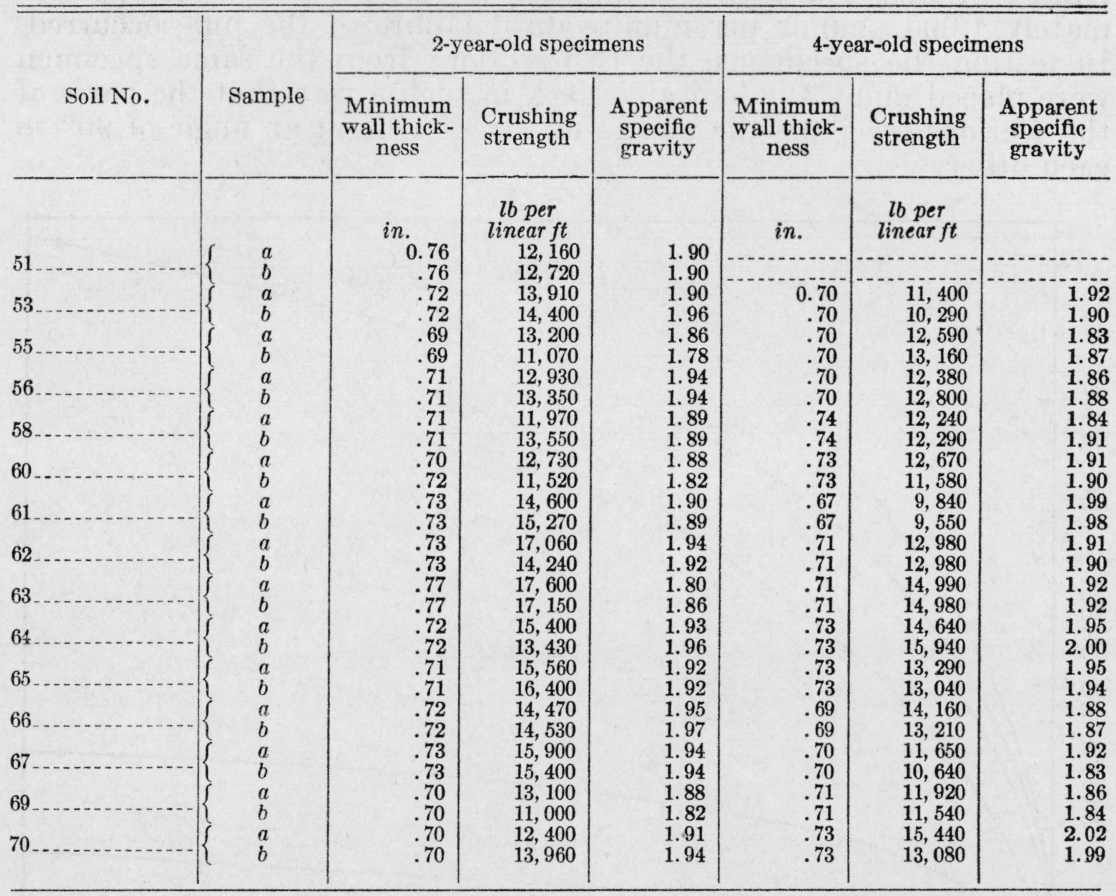

UNEXPOSED SPECIMENS

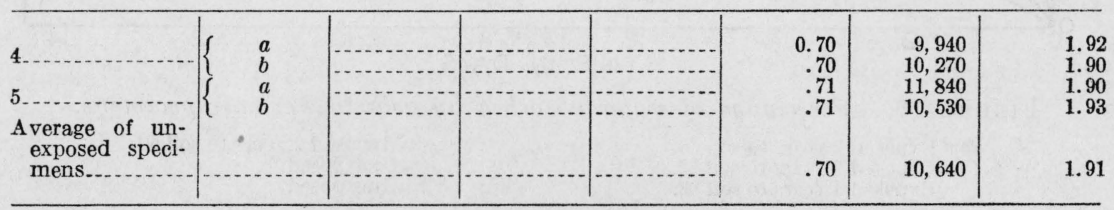

3. BURSTING TESTS

For the bursting tests the second of the pair of specimens removed from each test site at each inspection period was immersed in water for a minimum of 48 hours, and placed in the hydrostatic-pressure testing apparatus shown in figure 17. Internally fitting rubber cups were used to close the ends of the pipe. The apparatus was so designed that the pipe was not subjected to end compression during the test. After filling the pipe under test with water, the entrapped air was allowed to escape, and the pressure was increased at an approximate rate of 10 pounds per square inch per second until the pipe failed. The pressure gage employed was calibrated before the series of tests.

Many samples failed by a small piece splitting out at one end with the extension of a crack from this point along the entire length of the specimen. The others failed by cracking in one or two places along the entire length of the specimen. In all but three cases, a crack went through a hole, $3 / 16$-inch in diameter, near one end of the specimen, 
which was used to hold the identification tag. Here again there is no evident correlation between the bursting pressure and the age of the specimens either in individual soils or as any group of soils.

\section{APPARENT SPECIFIC GRAVITY}

Samples from the specimens upon which the crushing and bursting tests had been made were dried in an oven at about $110^{\circ} \mathrm{C}$ for 18 hours. After cooling to room temperature the weight of the dry sample was determined. The samples were then immersed in water for a period of 24 hours, and the weights in the wet condition were determined in air and when submerged in water. From the original weights of the dry samples and the volume of water displaced, the apparent specific gravities of the specimens shown in tables 32 and 33 were calculated. With few exceptions, the specific gravity of the specimens all fall between the values of 1.80 and 2.00 .

TABLE 33.-Results of bursting tests on the asbestos-cement specimens exposed to the various soils and on the unexposed specimens

\begin{tabular}{|c|c|c|c|c|c|c|}
\hline \multirow[b]{2}{*}{ Soil No. } & \multicolumn{3}{|c|}{ 2-year old specimens } & \multicolumn{3}{|c|}{ 4-year old specimens } \\
\hline & $\begin{array}{l}\text { Minimum } \\
\text { wall thick- } \\
\text { ness along } \\
\text { fracture }\end{array}$ & $\begin{array}{l}\text { Bursting } \\
\text { pressure }\end{array}$ & $\begin{array}{l}\text { Apparent } \\
\text { specific } \\
\text { gravity }\end{array}$ & $\begin{array}{l}\text { Minimum } \\
\text { wall thick- } \\
\text { ness along } \\
\text { fracture }\end{array}$ & $\begin{array}{l}\text { Bursting } \\
\text { pressure }\end{array}$ & $\begin{array}{l}\text { Apparent } \\
\text { specific } \\
\text { gravity }\end{array}$ \\
\hline 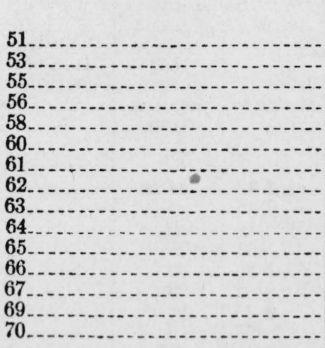 & $\begin{array}{r}\text { in. } \\
0.71 \\
.75 \\
.72 \\
.70 \\
.73 \\
.71 \\
.71 \\
.73 \\
.75 \\
.67 \\
.69 \\
.75 \\
.72 \\
.74 \\
.72\end{array}$ & $\begin{array}{r}\text { lb/in. }{ }^{2} \\
995 \\
1,140 \\
1,085 \\
995 \\
1,140 \\
1,010 \\
1,205 \\
1,095 \\
1,175 \\
1,100 \\
935 \\
1,215 \\
1,030 \\
1,255 \\
1,155\end{array}$ & $\begin{array}{l}1.89 \\
1.83 \\
1.87 \\
2.00 \\
1.76 \\
1.76 \\
1.92 \\
1.97 \\
1.84 \\
1.98 \\
1.76 \\
1.93 \\
1.86 \\
1.93 \\
1.80\end{array}$ & $\begin{array}{l}\text { in. } \\
0.71 \\
.72 \\
.73 \\
.71 \\
.72 \\
.71 \\
.70 \\
.74 \\
.74 \\
.70 \\
.71 \\
.73 \\
.68 \\
.77\end{array}$ & $\begin{array}{l}l b / \text { in. }^{2} \\
1,010 \\
1,100 \\
1,050 \\
925 \\
1,165 \\
1,065 \\
1,125 \\
1,150 \\
1,150 \\
1,070 \\
1,240 \\
1,105 \\
1,215 \\
1,285\end{array}$ & $\begin{array}{l}1.84 \\
1.77 \\
1.84 \\
1.90 \\
1.85 \\
1.94 \\
1.78 \\
1.88 \\
1.94 \\
1.84 \\
1.89 \\
1.82 \\
1.89 \\
1.84\end{array}$ \\
\hline
\end{tabular}

UNEXPOSED SPECIMENS

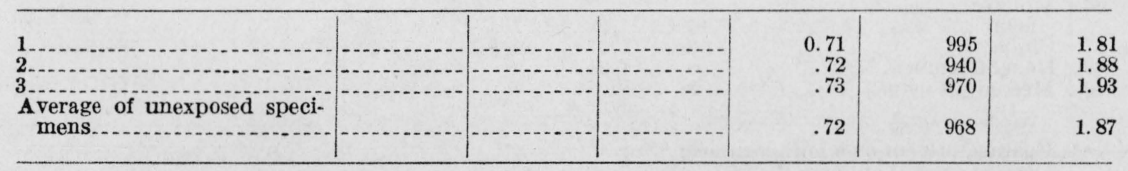

\section{COMPARISON OF THE DATA FROM VARIOUS TESTS}

Analysis of the data in tables 31 to 33 fails to bring out any correlation between any of the tests. The bursting- and crushing-test data for the specimens exposed for 2 and for 4 years do not show any evident differences. In some soils the 2-year specimens appear superior to the specimens exposed 4 years, and in other soils the reverse is true. No systematic differences between specimens exposed to different soil conditions can be detected.

However, the data do indicate that the asbestos-cement pipes generally gained strength during exposure to the soil, and that the softening observed on the outer layers of the specimens did not pene- 
trate deeply. For only two specimens are the values for the bursting pressure less than the maximum value of the unexposed specimens, and in only one soil are both values of the crushing strength for the exposed specimens less than the average value of the unexposed specimens. It is probable that during exposure of asbestos-cement pipe to the soil, a curing process takes place which tends to increase the strength of the pipe, but there is no indication that the pipe is any stronger after 4 years of exposure than after 2 years of exposure.

\section{METALLIC COATINGS}

\section{TINNED-COPPER TUBES}

Table 34 shows the loss of weight and maximum penetration of tinned-copper tube exposed for 4 years and similar data on deoxidized copper pipe exposed at the same test sites for 5 years. The table shows that in general the coating of tin temporarily reduced the rate of loss of weight but, in some cases at least, accelerated the rate of maximum penetration. The coating of tin was quite thin, and much of it had disappeared from the specimens buried in most of the soils.

TABLE 34.-Loss of weight and depth of maximum penetration of tinned-copper tubes exposed 4 years and copper pipe exposed 5 years

$M$, shallow metal attack as indicated by roughening of the surface.

$P$, definite pitting but no pits greater than 6 mils.

+ , one or both specimens contained holes caused by corrosion.

\begin{tabular}{|c|c|c|c|c|c|c|c|c|}
\hline \multicolumn{2}{|r|}{ Soil } & \multicolumn{3}{|c|}{ Tinned-copper tube } & \multicolumn{3}{|c|}{ Deoxidized copper pipe } & \multirow[b]{2}{*}{$\begin{array}{l}\text { Condi- } \\
\text { tion of } \\
\text { coating a }\end{array}$} \\
\hline No. & Type & Exposure & $\begin{array}{l}\text { Loss of } \\
\text { weight }\end{array}$ & $\underset{\substack{\text { Max- } \\
\text { penum } \\
\text { tion }}}{\text {. }}$ & Exposure & $\begin{array}{l}\text { Loss of } \\
\text { weight }\end{array}$ & $\begin{array}{c}\text { Max- } \\
\text { imum } \\
\text { penetra- } \\
\text { tion }\end{array}$ & \\
\hline & & Years & $0 z / f t^{2}$ & Mils & Years & $0 z / f t^{2}$ & Mils & \\
\hline 53 & Cecil clay loam.... & 4.01 & 0.086 & 7 & 5. 46 & 0.15 & 7 & \\
\hline 55 & Hagerstown loam & 3.90 & .11 & $\dot{M}$ & 5. 20 & .14 & $\dot{P}$ & 0 \\
\hline 56 & Lake Charles clay. & 3. 99 & .36 & 11 & 5. 44 & .51 & $P$ & 2 \\
\hline 58 & Muck ......... & 4.01 & .64 & 39 & 5. 50 & 1.56 & 12 & 2 \\
\hline 60 & Rifle peat...... & 3.98 & 4.54 & 42 & 5.25 & 3.82 & 28 & 3 \\
\hline 61 & Sharkey clay & 4.01 & 0.32 & b $18(2)$ & 5. 50 & 0.35 & 23 & 2 \\
\hline 62 & Susquehanna clay.- & 4.00 & .081 & $P$ & 5.47 & .26 & 9 & \\
\hline 63 & Tidal marsh & 4. 01 & 2.19 & c 14 & 5. 55 & 2.45 & $M$ & \\
\hline 64 & Docas clay & 3. 98 & 0.22 & 6 & 5. 22 & 2.22 & 9 & 2 \\
\hline 65 & Chino silt loam & 3.99 & .12 & $P$ & 5. 26 & 1.02 & 10 & \\
\hline 66 & Mohave fine gravelly & & & & & & & \\
\hline & loam & $\begin{array}{l}3.95 \\
3.98\end{array}$ & .23 & $\stackrel{P}{60+(2)}$ & 5. 28 & $\begin{array}{l}0.75 \\
9.33\end{array}$ & $\begin{array}{r}7 \\
54\end{array}$ & $\begin{array}{l}2 \\
3\end{array}$ \\
\hline $\begin{array}{l}67 \\
69\end{array}$ & Houghton muck & $\begin{array}{l}3.98 \\
3.98\end{array}$ & d 0.23 & ${ }_{P}^{60+(2)}$ & & & & \\
\hline 70 & Merced silt loam. & 3. 98 & .074 & 6 & & & & 1 \\
\hline
\end{tabular}

a 0 , Coating, present over entire surface;

1, Coating present on more than $50 \%$ of surface;

2 , Coating present on less than $50 \%$ of surface;

3 , Little or no coating left.

b (2) Indicates that 1 of the specimens was punctured after 2 years exposure.

-Uniform corrosion; no reference surface left.

dData for 1 specimen only.

Aside from the question of minimum thickness required for protection, a possible cause of failure of tinned-copper in soils is the reversal of potential of this couple. As tin is probably anodic to copper under all conditions, corrosion of tin would be expected to protect copper cathodically in the same manner that the corrosion of zinc protects the underlying steel in galvanized materials. The reversal of potential may be due to the formation of tin-copper alloys, which have been shown under certain condition to be more cathodic than copper [21]. 


\section{LEAD}

Table 35 shows the loss of weight and depth of maximum penetration of lead-coated and uncoated steel pipe exposed for 9 years. In all cases the coating reduced the loss of metal, and in 9 of the 13 soils the maximum penetration was also reduced. However, rather serious pitting occurred in all but one of the soils. Since lead, unlike zinc, is cathodic to iron and steel, protection of the underlying metal is accomplished by isolating the base metal from the enviornment. The life of the coating would then be expected to be determined largely by the corrodibility of lead under the conditions of exposure. Furthermore, if the corrosion of lead in soils takes the form of localized attack, or pitting, the potential difference between lead and the exposed steel would cause accelerated attack on the steel except under those conditions in which the steel might be polarized anodically. The average thickness of the lead coating was 1.44 mils. The corrosion for the three types of lead specimens shown in table 30 indicates that the protection provided by a coating of this thickness would be inadequate, since in all soils the maximum penetration of the lead pipe after 2 years' exposure to the same soil conditions exceeded the average thickness of the lead coating on the pipe specimens. It is doubtful that a lead coating of any reasonable thickness could be considered satisfactory for use in corrosive soils.

TABLE 35-Loss of weight and depth of maximum penetration of lead-coated and steel pipe exposed for 9 years

\begin{tabular}{|c|c|c|c|c|c|c|}
\hline \multicolumn{2}{|r|}{ Soil } & \multicolumn{2}{|c|}{ Lead-coated a $C A$} & \multicolumn{2}{|c|}{ Low-carbon steel $N$} & \multirow{2}{*}{$\begin{array}{l}\text { Condi- } \\
\text { tion of } \\
\text { coating }\end{array}$} \\
\hline No. & Type & $\begin{array}{l}\text { Loss of } \\
\text { weight }\end{array}$ & Penetration & $\begin{array}{l}\text { Loss of } \\
\text { weight }\end{array}$ & Penetration & \\
\hline $\begin{array}{l}53 \\
55 \\
56 \\
58 \\
59 \\
60 \\
61 \\
62 \\
63 \\
64 \\
65 \\
66 \\
67 \\
67\end{array}$ & $\begin{array}{l}\text { Cecil clay loam } \\
\text { Hagerstown loam } \\
\text { Lake Charles clay } \\
\text { Muck. } \\
\text { Carlisle muck } \\
\text { Rifle peat } \\
\text { Sharkey clay } \\
\text { Susquehanna clay } \\
\text { Tidal marsh } \\
\text { Docas clay } \\
\text { Chino silt loam } \\
\text { Mohave fine gravelly loam } \\
\text { Cinders }\end{array}$ & $\begin{array}{r}1.12 \\
0.76 \\
20.73 \\
14.85 \\
1.07 \\
\text { f } 7.07 \\
3.89 \\
3.64 \\
\text { - } 3.52 \\
2.10 \\
3.22 \\
3.39 \\
D\end{array}$ & $\begin{array}{l}41 \\
\quad 44 \\
\text { d } 145+(7) \\
\text { e } 91 \\
\text { f } 21 \\
50 \\
50 \\
59 \\
\text { e } 145+ \\
73 \\
72 \\
57 \\
145+(5,7)\end{array}$ & $\begin{array}{r}4.09 \\
3.82 \\
28.76 \\
16.24 \\
4.70 \\
16.72 \\
5.78 \\
6.65 \\
+9.03 \\
\text { g } D \\
12.86 \\
18.56 \\
\text { h } 58.39\end{array}$ & $\begin{array}{l}59 \\
59 \\
154+(7) \\
\text { e } 110 \\
\text { f } 40 \\
\text { ef } 27 \\
\text { f } 96 \\
\text { f } 87 \\
54 \\
154+(5,7) \\
112 \\
154+(5,7) \\
154+(2,5,7)\end{array}$ & $\begin{array}{l}2 \\
1 \\
3 \\
3 \\
1 \\
3 \\
2 \\
3 \\
2 \\
2 \\
2 \\
2 \\
3\end{array}$ \\
\hline
\end{tabular}

a This coating was 0.00144 inch thick, and contained 1 percent of tin.

b 1 , Coating on more than 50 percent of surface; 2 , coating on less than 50 percent of surface; 3 , little or no coating left.

- Data for only 1 specimen.

d + indicates that 1 or both specimens punctured. A number in parentheses after the pit depth indicates that 1 or both specimens were punctured in previous removals, e. g., (5) indicates a puncture after 5 years, etc.

- Uniform corrosion; no reference surface left on pipe.

Data for individual specimens differed from the average by more than 50 percent.

s $D=$ both specimens destroyed by corrosion.

h Data for 1 specimen only; the other specimen was destroyed.

\section{ZINC}

Zinc applied by the hot-dip method has been used extensively for the protection of small-diameter pipes under ground and its longcontinued use is evidence of its value under average conditions. In 1937 , steel specimens with a zinc coating having a weight of 3.08 ounces per square foot were buried at the 15 test sites previously men- 


\section{Journal of Research of the National Bureau of Standards}

tioned. The condition of these specimens after 4 years is reported in table 36. Figure 18 shows the galvanized and black-iron pipes that were buried as control specimens at three test sites. In all but one of the soils pitting began within the 4-year period, and the coating, including the zinc-iron alloy layer, was removed from at least half of the surface of the specimens. Under these conditions it seems probable that a zinc coating of the thickness and distribution of the one tested would not add more than 4 years to the life of a pipe exposed to the soils under observation. This result is inconsistent with the data of previous described tests [16], in which a coating of 2.82 ounces per square foot prevented pitting of the base metal in all but one of 47 soils over a 10-year period. Although certain of the soils in the more recent series of tests proved to be more corrosive than the soils in the older tests, it also is true that six soil conditions were duplicated in the two series of tests.

TABLE 36.-Loss of weight and depth of maximum penetration of galvanized and black iron pipe exposed 4 years

\begin{tabular}{|c|c|c|c|c|c|c|}
\hline & Soil & $\begin{array}{r}\text { Galvani } \\
3.08\end{array}$ & $\begin{array}{l}\text { ed pipe } \\
\mathrm{d} / \mathrm{ft}^{2}\end{array}$ & Black & ron pipe & \\
\hline No. & Type & $\begin{array}{l}\text { Loss of } \\
\text { weight }\end{array}$ & $\begin{array}{l}\text { Pene- } \\
\text { tration }\end{array}$ & $\begin{array}{l}\text { Loss of } \\
\text { weight }\end{array}$ & Penetration & \\
\hline & & $0 z / f t^{2}$ & Mils & $0 z / f t^{2}$ & Mils & \\
\hline $\begin{array}{l}53 . \\
55 .\end{array}$ & $\begin{array}{l}\text { Cecil clay loam } \\
\text { Hagerstown loam }\end{array}$ & $\begin{array}{r}1.39 \\
\text { b } 1.22\end{array}$ & & $\begin{array}{l}2.86 \\
2.60\end{array}$ & $\begin{array}{l}98 \\
50\end{array}$ & $\begin{array}{l}2 \\
2\end{array}$ \\
\hline 6. & Lake Charles clay. & 3. 89 & & 16. 03 & 104 & 2 \\
\hline & Muck & 5. 40 & b 21 & 8.78 & 46 & \\
\hline 60 & Rifle peat & 7.18 & 12 & 8. & b 38 & 3 \\
\hline 1 & Sharkey clay & 1. 46 & 12 & 4. 99 & 45 & 2 \\
\hline 62. & Susquehanna clay.. & 2. 28 & 9 & 4. 30 & 56 & 2 \\
\hline 63 & Tidal marsh....... & 2.15 & 10 & 9.20 & 38 & \\
\hline 64 & Docas ciay & 1. 58 & 9 & 5. 96 & 67 & 2 \\
\hline 65. & Chino silt loam & b 2.25 & 6 & 4. 56 & 59 & \\
\hline & Mohave fine gravelly loam & 3.32 & 8 & 12. 31 & e $145+(2)$ & \\
\hline 67 & Cinders & 5.40 & 45 & d 37.03 & $145+(2)$ & \\
\hline $69 \ldots$ & Houghton muck & 3.37 & 11 & 3.28 & 20 & 3 \\
\hline & Merced silt loam & 4.52 & 12 & b 9.72 & $118+$ & 3 \\
\hline
\end{tabular}

a 1, Coating on more than 50 percent of surface; 2 , coating on less than 50 percent of surface; 3 , little or no coating remaining.

b Data for individual specimens differed from each other by more than 50 percent.

- + indicates hole in 1 or both specimens caused by corrosion; (2) indicates that 1 or both specimens were punctured after 2 years' exposure.

d Data for 1 specimen; the other specimen was destroyed.

It was thought that the difference in behavior of the specimens having coatings of nominally the same thickness might possibly be ascribed to greater uniformity in distribution of the zinc coating in the earlier tests. However, measurements made with a Magne-gage on three unburied representative samples of the specimens from the earlier tests and on four similar specimens from the more recent tests showed that the variations in the thickness of the coatings on the pipe were no greater on one group than the other. Hence, the difference in behavior of the two specimens cannot be attributed to differences in uniformity of the zinc layers.

\section{NONBITUMINOUS ORGANIC COATINGS}

The value of the tests of organic coatings applied to short length of pipe under laboratory conditions is limited because many of ths failures of coatings in practice are attributable to injuries in the course 


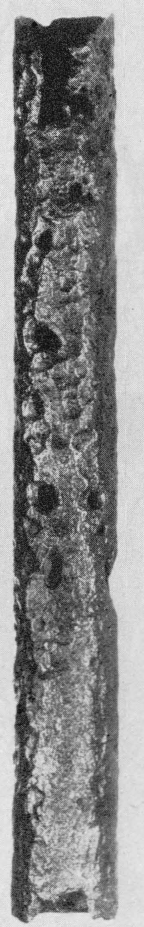

SI
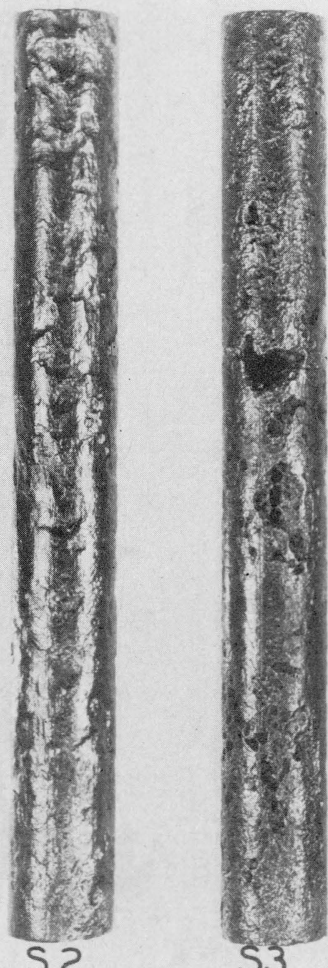
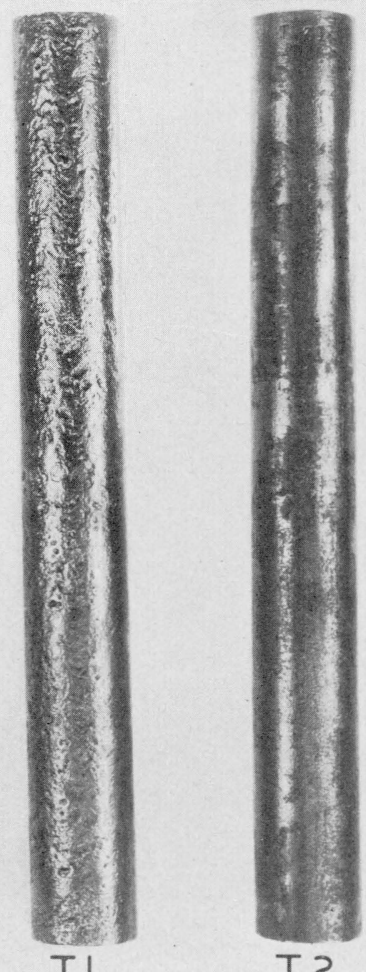

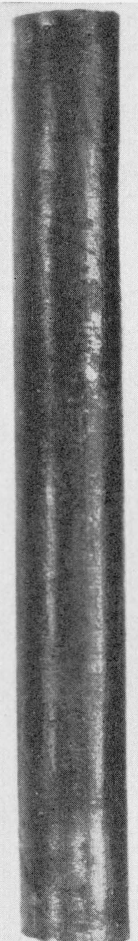

T3

FIGURE 18. - Black iron $(S)$ and galvanized $(T)$ pipes buried approximately 4 years in cinders (S1) and (T1), Lake Charles clay (S2) and (T2), and Mohave fine, gravelly loam (S3) and (TS). 


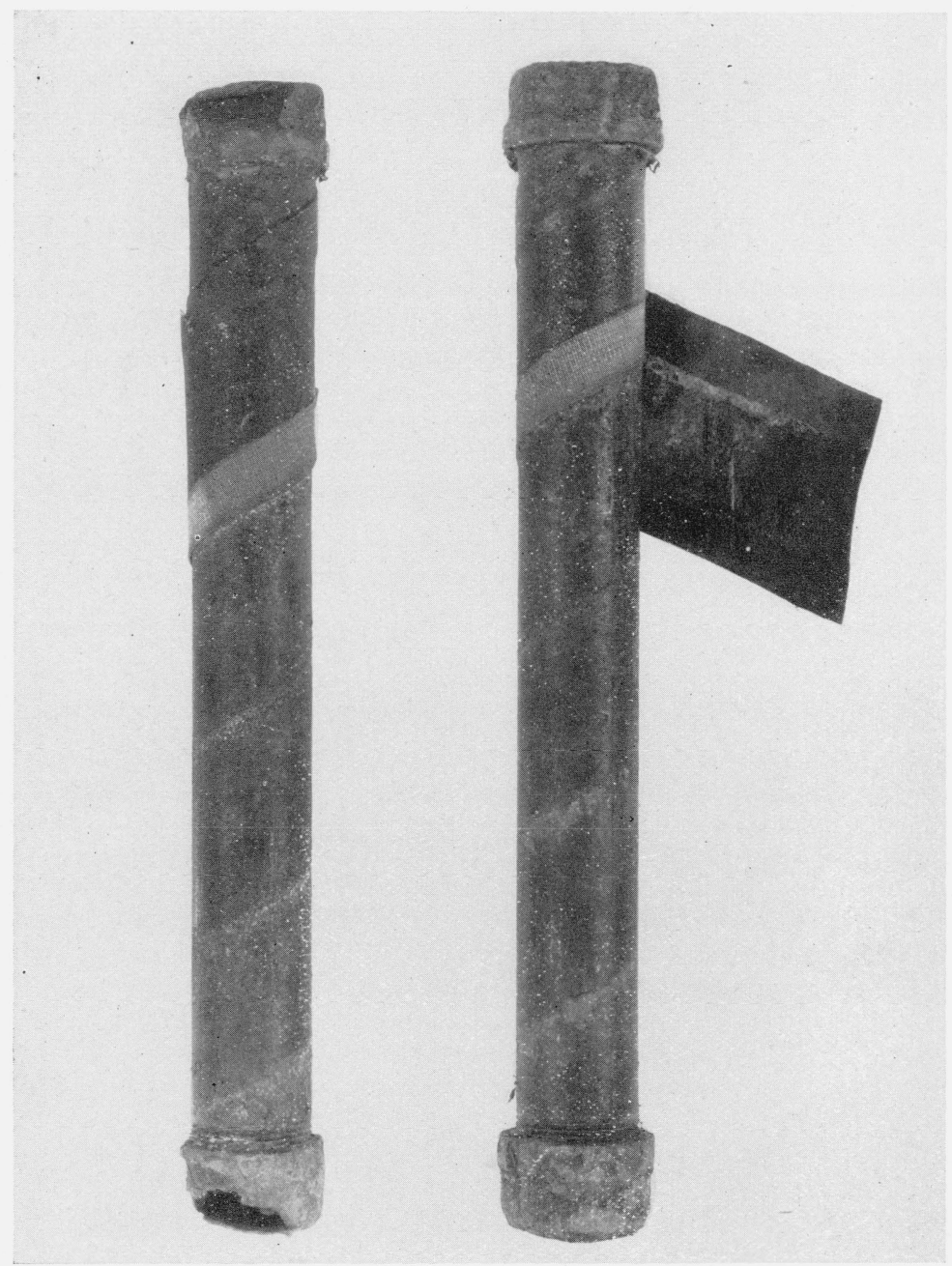

FiguRE 19.-Koroseal-coated pipe exposed for 4 years to cinders (soil 67). 
of the laying of the coated line. Moreover, it is difficult to produce a uniform coating in the field and even in the shop.

Such tests as are reported here are chiefly of value in that they show whether or not there is a possibility that a satisfactory coating could be made from the material tested. The tests may also suggest ways in which coatings could be improved. Most of the organic coatings reported on are either experimental or else materials which have been satisfactory under some conditions but are untried with respect to underground pipes. Few, if any, are in use in an experimental way on short sections of lines.

When the coated specimens were removed the coatings were examined for cracks, blisters, brittleness, change in color, and disbonding, as well as for rust spots and other evidence that corrosion had not been entirely prevented. Half of the coating was then removed and the extent of the corrosion, if any, recorded.

As it is frequently impossible to describe the condition of a coating accurately and briefly, and since the chief function of a coating for use in soil is to prevent corrosion, it has become customary to limit the report on organic coatings to a description of the condition of the pipe which they were intended to protect. This is not entirely satisfactory, as the depth of penetration is a function of the corrosiveness of the soil and probably nearly independent of the coating after the metal surface has been exposed. The seriousness of the corrosion may, however, indicate roughly the lapse of time since the surface became exposed if the corrosiveness of the soil is known. Even this is somewhat in doubt, since there is considerable evidence that under some conditions a faulty coating may accelerate corrosion either by localizing the corroding current or by modifying the differential aeration of the metal. Until recently many have regarded the usefulness of a coating as limited to the time during which it prevents corrosion. Experience has shown that with most coatings it is impractical to protect all points on a pipe surface because of accidents inherent in the application of the coating, the laying of the pipe line, root growth, or other injuries. Under most conditions it is practicable to protect electrically cross-country pipe lines, and possibly city pipe networks as well, by causing sufficient current to flow to the pipe. The cost of this method of preventing corrosion (cathodic protection) is roughly proportional to the current required and this is in turn proportional to the insulating properties of the protective coating. Thus a combination of protective coating and cathodic protection may be more economical than complete protection by either method alone. Under some conditions it may be more economical to permit a limited amount of corrosion than to prevent all of it. This, of course, depends on the losses assignable directly and indirectly to corrosion. It is from this point of view that pipe coatings should be judged.

\section{COATINGS EXPOSED FOR 9 YEARS}

The only information available descriptive of the coatings buried in 1932 was furnished by the manufacturers and is given below.

Coating B.-A synthetic rubber, stated to be an olefin polysulfide reaction product, was exposed in the form of sheets 10 by 5 by $1 / 4$ inches. Subsequently, a process was developed which permitted application of this material to pipes. Specimens coated by this

$604947-44-4$ 
process were exposed in 1939 and inspected in 1941. The condition of these coatings will be described in the section under coatings exposed for 2 years.

Coating C.- Two coats of porcelain enamel, one of which was acid-resisting. Thickness, 14 mils.

Coating D.-First coat, 23-percent solution of a rubber derivative in xylene; second and third coats, 30-percent solution of the rubber derivative in xylene; fourth coat, 20-percent solution of the rubber derivative in a mixture of turpentine and mineral spirits. Five percent of the solids was carbon black. Thickness, 10 mils.

Coating E.-Two applications of paint which differed in color. Neither the kind of pigment nor the kind of vehicle was specified. Thickness, 5 mils.

Coating F.-A semiplastic compound, which was applied cold with a brush, consisting of $4 \frac{1}{2}$ parts of treated cashew-nutshell oil, 3 parts of asbestos fiber, and $3 \frac{1}{2}$ parts of mineral turpentine substitute. Thickness, 6 mils.

Coating G.-A hard-rubber compound, containing rubber, sulfur, and an accelerator, cured to a bone-hard condition. Thickness, 90 mils.

Coating H.-A highly loaded hard-rubber stock that contained 30 percent of magnesium carbonate and approximately 15 percent of "white substitute." Thickness, 100 mils.

Coating J.-A synthetic resin varnish baked at $425^{\circ} \mathrm{F}$ for 30 minutes.

Coating $K .-\mathrm{A}$ paint containing highly chlorinated rubber, dissolved in a suitable solvent, to which may have been added drying oils, pigments, quartz meal, or carborundum. This coating was applied in Germany to pipe 1 inch in outside diameter. Thickness, 6 mils.

Coating W.-An experimental coating prepared as follows: The pipe was primed with a china-wood oil varnish containing zinc chromate and baked at $200^{\circ} \mathrm{F}$ for $1 / 2$ hour. A top coat of dehydrated china-wood oil, containing powdered mica and a catalyst, was molded on the pipe and heated to $200^{\circ} \mathrm{F}$ for 3 hours. Thickness, 170 mils.

Table 37 shows the condition of the pipe beneath the coatings exposed for 9 years. It will be noted that the two thick rubber coatings, $G$ and $H$, completely protected the pipes except at the ends where the coating had been injured mechanically. The porcelain enamel was nearly as good. It is probable that some of the corrosion reported was the result of injuries in handling of the specimens. It is claimed by the manufacturer that great strides have been made in resistance to chippage of porcelain enamel coatings during the past 9 years, and that the samples reported upon would not be truly representative of the type of porcelain enameling which would be obtainable today. However, as much greater care was taken than is practical with coatings on pipe lines, the corrosion resulting from injuries should not be entirely neglected.

The thick china-wood oil compound, $W$, prevented serious pitting in all soils. The fact that many of the coatings cracked and had a strong odor and the blistering of the priming coat indicate that the coating has deteriorated. However, because of its thickness it may continue to be helpful by isolating the pipe from the soil for several more years. The thin coatings $(D, E, F, J$, and $K$ ) have definitely deteriorated and are probably of little further use. Most of these coatings showed evidence of breakdown on earlier examinations. 
TABLE 37.-Condition beneath nonmetallic coatings exposed for 9 years

[Figures indicate depths of maximum pits in mils]

$\boldsymbol{E}$, ends corroded

$G$, ends rusted.

$H$, pipe punctured, $145+$ mils.

$M$, metal attack-pipe roughened by corrosion.

$R$, pipe rusted.

$P$, definite pitting but no pits greater than 6 mils.

$U$, no corrosion.

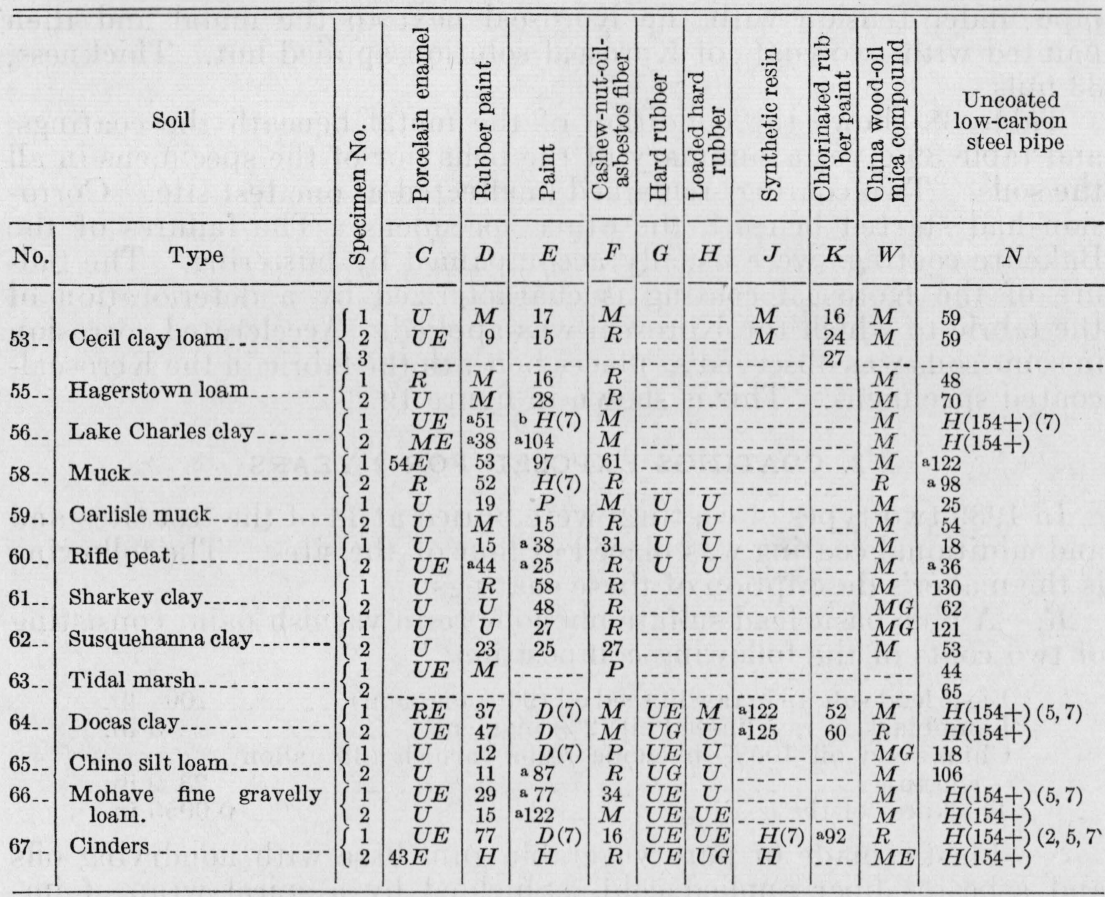

a Severe uniform corrosion; no reference surface; impossible to measure true penetration.

$\mathrm{b}$ The number in parentheses after the pit depth indicates that 1 or both specimens was punctured in the previous removal indicated by the number, (5) indicates that the pipe was punctured after 5 years, etc.

\section{COATINGS EXPOSED FOR 4 YEARS}

Five different nonmetallic coatings were buried in 1937. All of these coatings were of the phenol-formaldehyde (Bakelite) type with the exception of one which was of the polyvinyl chloride resin (Koroseal) type. All the coatings were applied to pipe about 12 inches long and $1 \frac{1}{2}$ inches in diameter. The descriptions of the coatings follow:

Coating L.-Two coats of a Bakelite varnish followed by two coats of another type of Bakelite varnish, each coat being baked after spraying. It appears as though the baking resulted in a fusion of the separate layers. Thickness, 7 mils.

Coating $M_{1}$. - Two coats of a Bakelite primer followed by two coats of a Bakelite chemically resistant aluminum paint. Each coat was sprayed and allowed to air-dry overnight. Thickness, 4 mils.

Coating $M_{2}$. - The same as coating $M_{1}$, except that it was applied to $2 \frac{1}{2}$ by 12 -inch plates instead of to pipe. Thickness, 3 mils.

Coating N.-A double layer of Bakelite-treated asbestos tape, the second layer overlapping the first, which was made to adhere to the pipe and to the first layer of tape by the use of an anticorrosive resin 
compound. A final protective coat was used over this tape, consisting of a spray coat of the same Bakelite chemically resistant aluminum enamel as used on the $M_{1}$ specimens. Thickness, 32 mils.

Coating P.-A fabric coated on one side with Koroseal to an overall thickness of 0.03 inch. The fabric was wrapped spirally on the pipe under tension with the Koroseal next to the metal and then painted with two coats of Koroseal solution applied hot. Thickness, 33 mils.

Table 38 shows the condition of the metal beneath the coatings, and table 39 gives a summary of the behavior of the specimens in all the soils. Two coatings remained unaffected at one test site. Corrosion had started beneath the other specimens. The failures of the Bakelite coatings were usually accompanied by blistering. The failure of the Koroseal coating is characterized by a deterioration of the fabric to which the Koroseal was applied. Accelerated corrosion in some soils was observed at places beneath the fabric in the Kerosealcoated specimens. This is shown in figure 19.

\section{COATINGS EXPOSED FOR 2 YEARS}

In 1939 two types of coatings were buried at 14 of the test sites and and additional coating was placed at four of the sites. The following is the maker's description of these coatings:

$R$.-A blue basic lead sulfate phenolic resin varnish paint consisting of two coats of the following composition:

Basic lead sulfate in raw linseed oil (90\% pigment) $100 \mathrm{lb}$.

Lampblack in raw linseed oil (2\% pigment) ........

Chinawood oil $100 \%$ phenolic resin varnish (33 gallon length) .

Thickness of the $23.9 \mathrm{lb}$.

S.-Plastic made of pure vegetable gum base with nondrying oils and asbestos fiber applied cold. Shielded by a spiral wrap of impregnated cotton fabric. Thickness, 0.044 inch.

T. Thiokol sprayed on; thickness, 0.035 inch. Two specimens of each type were removed from each soil in 1941. Table 40 shows the condition of each coated pipe after an exposure of 2 years. Only one specimen showed no sign of corrosion. 
TABLE 38.-Condition of metal beneath the Bakelite and Koroseal coatings exposed for 4 years

[A number indicates the maximum pit depth in mils]

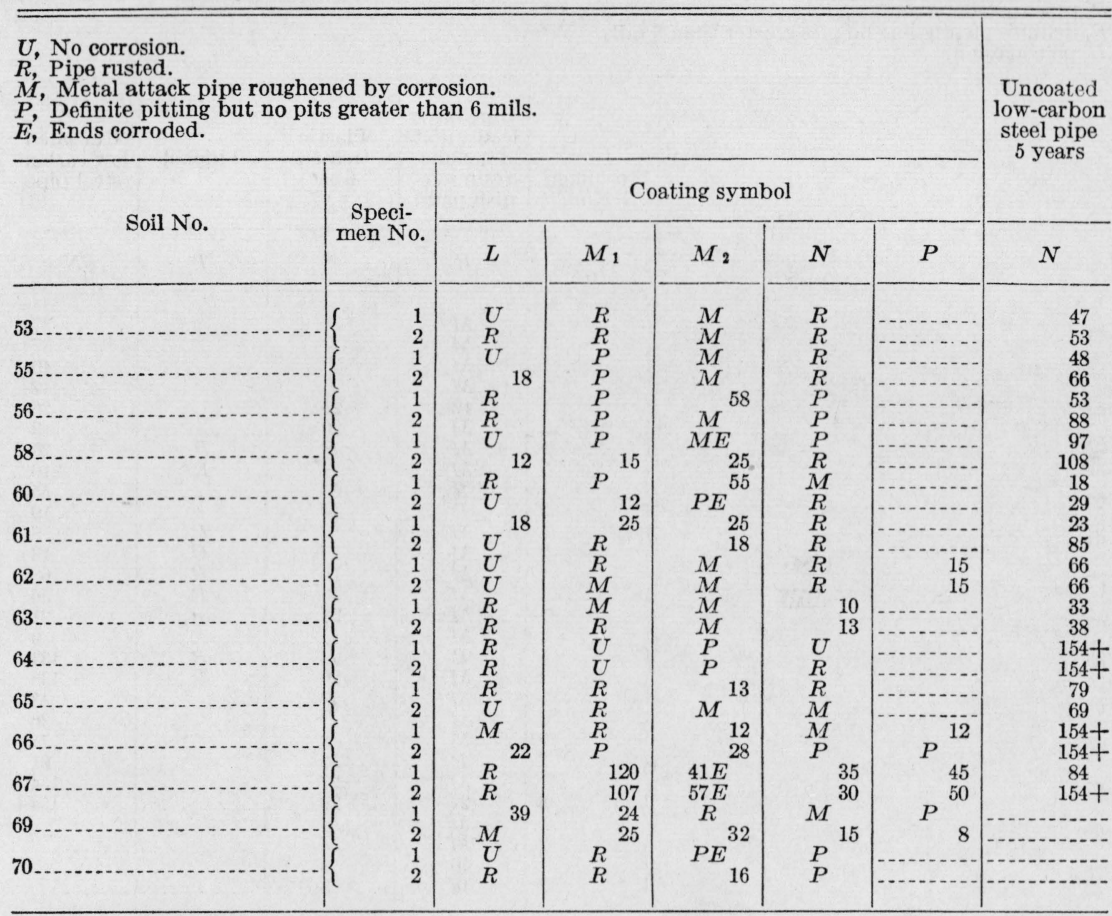

TABLE 39.-Summary of condition of coated pipe exposed for 4 years

[The figures in the table refer to the number of test sites. Data from specimens buried in 14 test sites, except for $P$ specimens, which were buried in 4 test sites. The poorer of the 2 specimens is reported on.

\begin{tabular}{|c|c|c|c|c|c|}
\hline \multirow{2}{*}{ Coating symbol } & \multicolumn{4}{|c|}{ Condition of pipe } & \multirow{2}{*}{$\begin{array}{c}\text { Coating } \\
\text { blistered }\end{array}$} \\
\hline & Unaffected & Rusted & Roughened & Pitted & \\
\hline $\begin{array}{l}M_{1} \\
M_{2}\end{array}$ & $\begin{array}{l}1 \\
1 \\
0 \\
0 \\
0\end{array}$ & $\begin{array}{l}8 \\
3 \\
0 \\
5 \\
0\end{array}$ & $\begin{array}{l}0 \\
2 \\
4 \\
2 \\
0\end{array}$ & $\begin{array}{r}5 \\
8 \\
10 \\
7 \\
4\end{array}$ & $\begin{array}{r}6 \\
13 \\
14 \\
-2.0\end{array}$ \\
\hline
\end{tabular}

- Poor bond between pipe and coating. 
TABLE 40.-Condition of coated pipe exposed for 2 years

[A number indicates the maximum pit depth in mils.

$M$, metal attack, pipe roughened by corrosion.

$R$, pipe rusted.

$P$, definite pitting but no pits greater than 6 mils.

$U$, no corrosion.

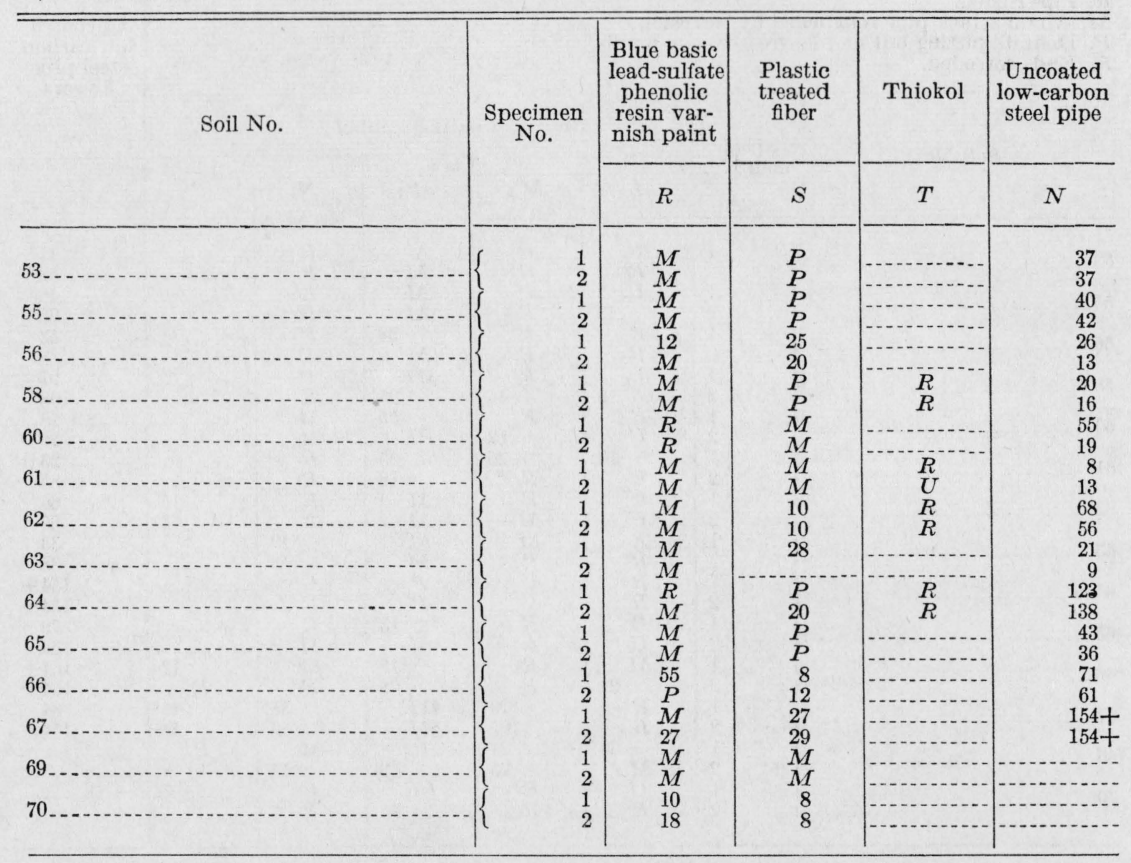

The blue basic lead sulfate coating $R$, blistered and became scaly. There was little adhesion between the plastic coating $S$ and the pipe. The entire surface of each pipe coated with the plastic rusted. Soil indented and adhered to the coating. In most soils the coating appeared to have rotted.

On each specimen of Thiokol-coated pipe there was a network of fine cracks, which usually did not permit water to reach the pipe. In a number of cases the coating was chipped, possibly by the formation of rust beneath the coating.

\section{SUMMARY}

This report deals with the conditions of a large variety of ferrous and nonferrous materials and a considerable number of metallic and organic coatings exposed from 2 to 9 years to 14 corrosive soils. The primary purpose of the tests was to assist manufacturers in obtaining data on the effectiveness of their corrosion-resistant materials with respect to corrosive soils and to aid them in the development of better materials.

Although the addition of small percentages of certain alloying elements improves somewhat the resistance of ferrous alloys to some soil conditions, the improvement cannot be considered significant. Ferrous materials must contain very considerable quantities of 
nickel, chromium, or other elements if they are to withstand the action of severely corrosive soils. The behavior of 4- to 6-percentchromium steel plates exposed 4 years is in agreement with the behavior of 5-percent-chromium steel tubes exposed 9 years in that lower loss of weight and deeper pitting were observed in comparison with plain steel.

Under most corrosive soil conditions, copper and alloys high in copper are very resistant to corrosion, and the corrodibility appears to increase with the amount of zinc in the alloy. Since dezincification plays an important role in the corrosion of brasses, weight losses and pit-depth measurements do not give a true estimation of the extent of corrosion taking place in some of the brass specimens.

The addition of 0.08 percent of arsenic to Muntz metal did not prevent dezincification.

Bursting and crushing tests indicate that asbestos-cement pipe gained somewhat in strength after exposure to the soil for 4 years.

The two lead alloys corroded at about the same rate as chemical lead. The extent of corrosion on the lead-coated specimens indicates that a lead coating of any reasonable thickness can not be considered satisfactory for use in corrosive soils.

A coating of tin over copper gave little protection over a period of 4 years.

Several experimental coatings greatly reduced corrosion over periods of from 4 to 9 years. Among these are a vitreous enamel, two thick rubber coatings, a thick molded coating of china-wood oil and mica, and a thin baked-on Bakelite coating (4 years).

The thin coatings, of which there were several, reduced corrosion but showed distinct signs of deterioration and cannot be considered adequate for severe soil conditions.

\section{REFERENCES}

[1j K. H. Logan, Soil-corrosion studies, 1934: Rates of loss of weight and pitting of ferrous specimens, J. Research NBS 16, 431 (1936) RP883.

[2] K. H. Logan, Soil-corrosion studies, 1939: Ferrous and nonferrous corrosionresistant materials, J. Research NBS 28, 379 (1942) RP1460.

[3] K. H. Logan, Soil-corrosion studies, 1937: Corrosion-resistant materials and special tests, J. Research NBS 23, 515 (1939) RP1250.

[4] K. H. Logan and R. H. Taylor, Soil-corrosion studies, 1932. Rates of loss of weight and pitting of ferrous and nonferrous specimens and metallic protective coatings, BS J. Research 12, 119 (1934) RP638.

[5] S. P. Ewing, Field tests of pipe coatings, Proc. Am. Gas Assn. 62\%, 656 (1936).

[6] K. H. Logan, API Pipe coating tests, Proc. Am. Petroleum Inst. 21 (IV) 32 (1940).

[7] K. H. Logan, Conclusions based on the American Petroleum Inst. coating tests, Petroleum Engr. 12, No. 9, 100 (1941).

[8] K. H. Logan, S. P. Ewing, and C. D. Yeomans, Bureau of Standards soilcorrosion studies. I, Soils, materials, and results of early observations, BS Tech. Pap. 22, 447 (1927-28) T368.

[9] R. F. Hadley, Studies in microbiological anaerobic corrosion, Proc. Am. Gas Assn. 764 (1940).

[10] I. A. Denison, Electrolytic measurements of the corrosiveness of soils, J. Research NBS 17, 363 (1936) RP918.

[11] I. A. Denison, Electrolytic behavior of ferrous and nonferrous metals in soilcorrosion circuits, Trans. Electrochem. Soc. 81, 435 (1942).

[12] R. M. Burns, The corrosion of metals I, Bell System Tech J. 15, 28 (1936).

[13] R. H. Brown and R. B. Mears, The electrochemistry of corrosion, Trans. Electrochem. Soc. 74, 495 (1938).

[14, K. H. Logan, Engineering significance of National Bureau of Standards soilcorrosion data, J. Research NBS 22, 109 (1939) RP1171. 


\section{Journal of Research of the National Bureau of Standards}

[15] K. H. Logan, Soil-corrosion studies, 1934: Rates of loss of weight and penetration of nonferrous materials, J. Research NBS 1\%, 781 (1936) RP945.

[16] Kirk H. Logan and Scott P. Ewing, Fisld tests of nonbituminous coatings for underground use, J. Research NBS 18, 361 (1937) RP982.

[17] K. H. Logan, Soil-corrosion studies, 1939: Coatings for the protection of metals underground, J. Research NBS 28, 57 (1942) RP1446.

[18] M. Romanoff, Effect of aeration on the hydrogen-ion concentration of an anaerobic soil, Fifth National Bureau of Standards Soil Corrosion Conference, 1943.

[19] R. J. McKay and R. Worthington, Corrosion resistance of metals and alloys (Rheinhold Publishing Corporation, New York, N. Y., 1936).

[20] H. L. Burghoff, by correspondence.

[21] P. D. Merica, Structure of the coating on tinned sheet copper in specific case of corrosion, BS Tech. Paper 9 (1917) T90.

Washington, April 1, 1944. 